\title{
Behavioral inhibition as a risk factor for the development of childhood anxiety disorders
}

Citation for published version (APA):

van Brakel, A. M. L. (2007). Behavioral inhibition as a risk factor for the development of childhood anxiety disorders. [Doctoral Thesis, Maastricht University]. Datawyse / Universitaire Pers Maastricht. https://doi.org/10.26481/dis.20070721ab

Document status and date:

Published: 01/01/2007

DOI:

10.26481/dis.20070721ab

Document Version:

Publisher's PDF, also known as Version of record

\section{Please check the document version of this publication:}

- A submitted manuscript is the version of the article upon submission and before peer-review. There can be important differences between the submitted version and the official published version of record.

People interested in the research are advised to contact the author for the final version of the publication, or visit the DOI to the publisher's website.

- The final author version and the galley proof are versions of the publication after peer review.

- The final published version features the final layout of the paper including the volume, issue and page numbers.

Link to publication

\footnotetext{
General rights rights.

- You may freely distribute the URL identifying the publication in the public portal. please follow below link for the End User Agreement:

www.umlib.nl/taverne-license

Take down policy

If you believe that this document breaches copyright please contact us at:

repository@maastrichtuniversity.nl

providing details and we will investigate your claim.
}

Copyright and moral rights for the publications made accessible in the public portal are retained by the authors and/or other copyright owners and it is a condition of accessing publications that users recognise and abide by the legal requirements associated with these

- Users may download and print one copy of any publication from the public portal for the purpose of private study or research.

- You may not further distribute the material or use it for any profit-making activity or commercial gain

If the publication is distributed under the terms of Article $25 \mathrm{fa}$ of the Dutch Copyright Act, indicated by the "Taverne" license above, 
BEHAVIORAL INHIBITION as a RISK FACTOR for the DEVELOPMENT of CHILDHOOD ANXIETY DISORDERS 


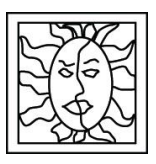

(C) A M L van Brakel, MAASTRICHT 2007

OMSLAGONTWERP \& LAYOUT: designocima ${ }^{\circledR}$, www.designocima.com

DRUK: Datawyse

ISBN: 9789080771581

All rights reserved. Illustrations and brief excerpts from this publication may be used for scientific and educational purposes provided that the source is acknowledged. 


\section{BEHAVIORAL INHIBITION as a RISK FACTOR for the DEVELOPMENT of CHILDHOOD ANXIETY DISORDERS}

\section{PROEFSCHRIFT}

ter verkrijging van de graad van doctor aan de Universiteit Maastricht, op gezag van de Rector Magnificus, Prof. mr. G.P.M.F. Mols, volgens het besluit van het College van Decanen, in het openbaar te verdedigen op donderdag 21 juni 2007 om 14:00 uur

door ANNA MARIA LOUISA VAN BRAKEL 


\section{PROMOTORES}

Prof. dr. Peter Muris

Prof. dr. Arnoud Arntz

\section{BEOORDELINGSCOMMISSIE}

Prof. dr. H. L. G. J. Merckelbach (voorzitter)

Prof. dr. A. Jansen

Dr. D. Bernstein

Prof. dr. M. Westenberg (Universiteit Leiden) 


\section{CONTENTS}

CHAPTER I Introduction. Behavioral inhibition as a risk factor for the development of childhood anxiety disorders: A review

CHAPTER 2 Study 1. Relations between parent- and teacher-reported behavioral inhibition and behavioral observations of this temperamental trait

CHAPTER 3 Study 2. A brief scale for measuring "behavioral inhibition to the unfamiliar" in children

CHAPTER 4 Study 3. Eye blink startle responses in behaviorally inhibited and uninhibited children

CHAPTER 5 Study 4. A multifactorial model for the etiology of anxiety in non-clinical adolescents: Main and interactive effects of behavioral inhibition, attachment, and parental rearing

CHAPTER 6 Study 5. Behavioral inhibition as a risk factor for the development of childhood anxiety disorders:

A longitudinal study

References

Dankwoord

Curriculum Vitae 



\section{CHAPTER 1}

General Introduction:

Behavioral inhibition as a risk factor for the development of childhood anxiety disorders: A review 


\begin{abstract}
Behavioral inhibition is a character trait that refers to the tendency of some children to respond to new, unfamiliar situations with extreme shyness and withdrawal. Behavioral inhibition is a construct that is largely based on observations in the laboratory and is considered to be a genetically transmittable temperamental disposition that may be a risk factor for the development of anxiety disorders in children. Anxiety disorders are among the most common psychiatric diagnoses in children and adolescents. This chapter first discusses the construct of behavioral inhibition in more detail and then examines the question whether behavioral inhibition is a risk factor for the development of pathological fear and anxiety in youths. The moderating factors that may determine whether or not behavioral inhibition results in the development of anxiety disorders in children are also addressed. A review of current research will show that behavioral inhibition is a relatively stable character trait and most likely a risk factor for the development of anxiety disorders. Environmental factors appear to play a prominent moderating role in this process. Factors related to the family environment, such as parenting style and quality of attachment, could be particularly important for determining whether or not children develop anxiety disorders. The final section of this chapter discusses some limitations of behavioral inhibition research.
\end{abstract}




\section{INTRODUCTION}

Behavioral inhibition has been proposed to be a character trait that refers to the tendency of some children to respond to new, unfamiliar situations with extreme shyness and withdrawal (Kagan, Reznick \& Snidman, 1988). Behavioral inhibition is considered to be a largely genetic temperamental trait that may be a risk factor for the development of anxiety disorders in children. Anxiety disorders are among the most common psychiatric diagnoses in children and adolescents (Craske, 1997), and are associated with numerous negative emotions, cause social dysfunction and may have a negative effect on school performance (Biederman, Rosenbaum, Chaloff \& Kagan, 1995). Nevertheless, externalizing (behavioral) problems have long dominated research into psychiatric problems in children and adolescents. This is partly caused by the simple fact that externalizing problems in school-going children are much more noticeable and cause more immediate difficulties to the environment than the withdrawn, anxious behavior of children with internalizing problems (including anxiety disorders; Rubin \& Mills, 1991). Furthermore, it was long thought that anxieties in childhood were only temporary, developmental phenomena. With the publication of DSM-III (American Psychiatric Association, 1980), anxiety disorders in children were given a prominent place in psychiatric diagnostics (Last, Perrin, Hersen \& Kazdin, 1996). Partly as a result of this, interest in anxiety disorders in children has increased greatly over the past two decades, as has the amount of research conducted into these disorders. Research on childhood anxiety disorders focuses on several areas, including etiology. Studies in this domain try to find out which factors contribute to the development of anxiety disorders in children and adolescents. Laboratory observations conducted during the 1980s lead to the formulation of the construct of behavioral inhibition as an important possible explanatory factor for the development of anxiety disorders. However, the construct of behavioral inhibition by definition already overlaps with the diagnostic criteria. For instance, 'withdrawal behavior in new, unfamiliar situations' can be viewed as avoidance behavior, which of course is an essential component of various anxiety disorders. A degree of correlation between behavioral inhibition in childhood and anxiety at a later age would then be evident in advance. Nevertheless, it is important to investigate this relationship further, as anxiety disorders cannot yet be diagnosed at a young age. Behavioral inhibition can then serve as a tool to detect, at an early stage, children who are at an increased risk of developing an anxiety disorder. This chapter will first discuss the construct of behavioral inhibition in more detail, paying particular attention to the different 
measuring methods and the stability, context-specificity and physiological correlates of this temperamental characteristic. The question whether behavioral inhibition is a risk factor for the development of pathological fear in children will then be addressed. In this context, several moderating factors that may determine whether or not behavioral inhibition results in the development of anxiety disorders in children will be discussed. These may include factors related to the family environment which have been shown to play a role in the development of anxiety disorders, such as mother-child attachment and parenting style. The various risk factors are then presented in an integrated model. Finally, some limitations of behavioral inhibition research are critically discussed. The conclusion will address the value of the construct of behavioral inhibition in explaining the development of anxiety disorders.

\section{METHODS FOR MEASURING BEHAVIORAL INHIBITION}

The level of behavioral inhibition can be determined by observing children in new, unfamiliar (laboratory) situations. Behavioral inhibition in older children and adolescents can be measured using a self-reporting questionnaire. The specific situations that may be used to elicit inhibited behavior and the questionnaires used are discussed below.

According to Kagan et al. (1988), children with an inhibited temperament respond with extreme shyness, withdrawal, introversion, and excessive sympathetic arousal to new stimuli, new events, and especially new people. At the start of a longitudinal study, Garcia Coll, Kagan and Reznick (1984) brought children of approximately two years of age $(N=117)$ to the laboratory. Before these lab sessions, 305 prescreen telephone interviews were conducted. Based on these interviews, 160 infants were selected as they were classified as either inhibited or uninhibited. Of this sample, eventually 117 children came to the laboratory. These children were observed while they were exposed to unfamiliar objects and individuals. The behavioral observation consisted of six standardized procedures. One of these procedures was a meeting with an unfamiliar adult. The trial leader left the room and an unfamiliar woman entered. The woman sat on a chair, avoiding any form of communication with the child or the mother for approximately 30 seconds. The woman then called the child by his or her name and asked the child to come sit on the floor and carry out a number of assignments involving the toys present in the room. Another procedure concerned the child's reaction to an unfamiliar object. The trial leader came back into the room and opened the curtains 
in a corner of the room, revealing a talking robot of 60 by 15 centimeters. The child was then encouraged to investigate the robot. The degree of behavioral inhibition of each child was ascertained through observation. This was done by noting the time that elapsed before the child approached the unfamiliar person or object (the so-called 'latency period'). The amount of time that the child spent near his or her mother was also noted. Based on the data observed, the children were then assigned to one of the following two groups. The first group consisted of behaviorally inhibited children who were continually shy, anxious or withdrawn during the abovementioned laboratory procedures. The second group consisted of children who were continually sociable, talkative and spontaneous, and who could best be described as non-inhibited (Garcia Coll et al., 1984). The two groups were observed on three subsequent occasions, namely at age 3.5 to 4 , at age 5.5 and at age 7.5 (Kagan et al., 1988). The specific laboratory procedures that were initially used were changed, as older children react with shyness to different situations than younger children. According to Kagan (1989), a confrontation with an unfamiliar adult is a powerful stimulus for a two-year-old child and can be used to evaluate the child's level of inhibition. At age 4, however, it would be better to use an unfamiliar child of the same age as a stimulus, while at age 7 an entire group of unfamiliar children would be needed to detect differences in the behavior of inhibited and non-inhibited children.

Various studies have shown that the level of behavioral inhibition can also be determined by asking youths to complete a self-reporting questionnaire (Gest, 1997; Muris, Merckelbach, Wessel, \& Van de Ven, 1999; Muris, Merckelbach, Schmidt, Gadet, \& Bogie, 2001). An example of such a questionnaire is the Behavioral Inhibition Scale (BIS; see Muris et al., 1999), which is composed of two parts. The first part consists of four items measuring the social inhibition (or lack thereof) of the child: shyness ("I am shy when I have to talk to someone I don't know"), communication ("I find it easy to talk to someone I don't know"), fear ("I feel nervous when I have to talk to someone I don't know") and laughter ("When I talk to someone I don't know, I feel good and I am able to laugh"). In the second part of the questionnaire, the child/adolescent is presented with three descriptions from which they must select the one that best describes them. The descriptions represent a high level of behavioral inhibition ("For as long as I can remember, I have been shy when I have to talk to people I don't know. I feel nervous, I find it difficult to laugh, and I don't know what to say"), a low level of behavioral inhibition ("For as long as I can remember, I have found it easy to talk to people I don't know. I feel good, I am able to laugh and I know exactly what to say"), and an average level of behavioral 
inhibition ("I fall somewhere between 1 and 2 "), respectively. This questionnaire is also available in a version for parents and teachers.

Behavioral inhibition can already be established at a very early age (from 4 months onwards) by means of behavioral observations in the laboratory. Research has indicated that questionnaires such as the BIS can be used to measure behavioral inhibition in older children and adolescents (from age 8).

\section{STABILITY OF BEHAVIORAL INHIBITION OVER TIME}

In order to determine whether a particular behavior of the child forms part of its temperament and is not displayed accidentally due to the situation in which the child finds itself, it is necessary to ascertain whether this behavior remains stable over a longer period of time and over a wide range of situations. In other words, do children who are classified as inhibited at the age of 2 still act withdrawn and anxious in response to new situations several years later? And what about children who are sociable and exploratory in response to unfamiliar situations at the age of 2 ?

The study by Kagan et al. (1988) showed that $75 \%$ of the children who were classified as inhibited or non-inhibited at the age of 2 still displayed the same behavior six years later when they were exposed to a new situation or met new people. To be exact, $59 \%$ of the children classified as inhibited at the age of 2 remained inhibited until the first follow-up at age 4 and only one of the originally uninhibited sample was more inhibited at the follow-up (see also Scarpa, Raine, Venables, \& Mednick, 1995; and for an overview Turner, Beidel, \& Wolff, 1996). The results obtained by Gest (1997) suggest that this stability even extends into adolescence and early adulthood.

Physiological indicators may be useful in order to distinguish between children who are temperamentally shy and withdrawn in response to a new situation (and whose behavior will remain stable in this respect) and children who respond to that same new situation with shyness and withdrawal due to environmental factors (and whose behavior will therefore not remain stable in this respect). Based on the findings of Kagan et al. (1988), Turner et al. (1996) suggest that heart rate may be of predictive value in the detection of children who remain stable in displaying inhibited behavior. Eighty percent of the children in the Kagan study who retained their inhibited status, displayed a stable, high heart rate at age 2, in contrast to the children who appeared less inhibited during the same follow-up. Even infants display certain behavior that can predict whether or not they will display inhibited behavior in 
response to new, unfamiliar situations when older, i.e. as toddlers. A high level of motor activity and crying in response to new stimuli at the age of 4 months are predictive for behavioral inhibition at age 2 (Kagan \& Snidman, 1991). In a study of a very large group of children ( $N=1795)$, Scarpa et al. (1995) also demonstrated that behavioral inhibition is quite stable over time. It also appears that if children retain their inhibited status between the ages of 3 and 8 , it is highly likely that they will still display the same behavior at age 11, in contrast to children who display varying levels of inhibition between the ages of 3 and 8 . This seems to lend support to the proposition that behavioral inhibition is a temperamental trait present in a substantial minority of children.

Behavioral inhibition appears to be a stable character trait in approximately two-thirds of children who are classified as inhibited at a young age. The following indicators are predictive for stable behavioral inhibition: a high level of motor activity and crying in response to new stimuli in 4-month-old babies, and a high, stable heart rate in children of 2 years or older.

\section{CONTEXT-SPECIFICITY OF BEHAVIORAL INHIBITION}

Are behaviorally inhibited children extremely shy, withdrawn and anxious in every new situation? Or are there specific new situations that elicit this style of response? These questions are important with a view to the reliability and validity of the methods used to measure behavioral inhibition, determine the stability of behavioral inhibition, and investigate the relationship between behavioral inhibition and anxiety disorders.

In the study by Kagan et al. (1988), social and non-social contexts were combined to obtain an impression of the level of inhibition. This entailed confronting the children with unfamiliar objects (non-social cues), but also with unfamiliar adults (social stimuli). However, several studies show that the level of behavioral inhibition depends on the situation in which it is measured. Stevenson-Hinde and Glover (1996) discovered to their surprise that a small number of children had to be classified in the 'extremely shy' group based on observations at home, while those same children fell into the 'not shy at all' group when observed in the laboratory. Rubin, Hastings, Stewart, Henderson, and Chen (1997) demonstrated that a 'system of social behavioral inhibition' can exist independently of a 'system of non-social behavioral inhibition'. They concluded that inhibited behavior in early childhood can take different forms and may therefore have different functions. According to these researchers, each form of inhibited behavior reflects the activity of a different 
behavior system. This means that toddlers who experience a high level of all forms of inhibition display consistent (inhibited) behavior in different (i.e., social and non-social) contexts. A toddler may also be extremely wary in the company of adult strangers, but not necessarily inhibited in a non-social context.

It appears that care must be taken when determining the level of behavioral inhibition. Behavior, in this case behavioral inhibition, should be observed in different situations, especially given the fact that the level of behavioral inhibition is context-dependent. If researchers fail to do so, there is a substantial risk of misclassification.

\section{PHYSIOLOGICAL CORRELATES OF BEHAVIORAL INHIBITION}

Specific behavioral profiles, such as behavioral inhibition, are said to be determined by hereditary physiological processes that predispose children to display specific emotions and behaviors (Kagan, Snidman, \& Arcus, 1993). These researchers therefore propose that certain innate physiological characteristics may be expressed at the behavioral level in the form of behavioral inhibition. Behavioral inhibition is then viewed as a child's particular temperament, comprising behavioral and (hereditary) biological components. If this is the case, what are the physiological correlates of behavioral inhibition? And are these physiological processes indeed hereditary?

Numerous studies support the hypothesis that behaviorally inhibited children are born with a low threshold for limbic-hypothalamic arousal. As a result, unexpected changes in the environment or new situations which are difficult to process very quickly produce a chain of physiological activity (Garcia Coll et al., 1984; Kagan et al., 1988; Snidman, 1989; see Adamec \& Stark-Adamec, 1989 for corresponding results from animal studies). Indications that this is the case include the higher, more stable heart rate of behaviorally inhibited children when processing unfamiliar information, in contrast to non-inhibited children. Schmidt, Fox, Rubin et al. (1997) put forward the hypothesis that high cortisol levels in behaviorally inhibited children lead to an increase in the secretion of corticotropin-releasing hormone $(\mathrm{CRH})$ by the amygdala. The increased secretion of this stress hormone causes permanent changes in the amygdala, leading to an aggravation of the anxieties experienced by these children. It has also been shown that inhibited children are characterised by a lack of activation in the left-frontal 'approach' system that is probably caused by increased activity in the limbic system (Davidson, 1993, see also 
Calkins, Fox, \& Marshall, 1996; Kalin, 1997). The possibility that inhibited children are not born with a low threshold for limbic activation, but that early experiences change their neuroendocrine system (Van Doornen, 2000; LeDoux, 1997) cannot be excluded. Support for this hypothesis is provided by animal studies (Liu, Diorio, Tannenbaum et al., 1997; Sapolsky, 1997). However, genetic analyses show that the relationship between social inhibition at age 1, 1.5, 2, and 3, and internalizing problems at age 4 in twins is largely due to genetic factors (Schmitz, Fulker, Plomin et al., 1999). This supports the hypothesis that behavioral inhibition is mainly the result of a genetic physiological predisposition.

Broadly speaking, the preceding can be summarized as follows: a substantial minority of children are born with a biological vulnerability to sympathetic arousal that is expressed in extreme withdrawal, that is to say, 10 to $15 \%$ of children have an area in the brain with a very low activation threshold. The region of the brain referred to here comprises the hypothalamus and the limbic system, particularly the amygdala. The limbic system is strongly associated with emotions, the amygdala is concerned with the processing of fear-related stimuli, while the hypothalamus plays a role in stress, among other functions. When inhibited children are confronted with new, unfamiliar situations, this region of the brain is immediately activated, leading to an increased heart rate, minimal heart rate variability, pupil dilation and high levels of the stress hormone cortisol. Genetic analyses seem to suggest that this vulnerability is hereditary.

Behavioral inhibition as a risk factor for the development of anxiety disorders

A significant portion of children and adolescents develop an anxiety disorder. Costello and Angold (1995) provide an overview of 16 epidemiological studies on the prevalence of anxiety disorders in children and adolescents, conducted between 1988 and 1995. These studies yielded (life-time) prevalence rates ranging from $5.7 \%$ to $17.7 \%$, with more than half of the studies yielding a rate of more than $10 \%$. The overview shows that the prevalence of anxiety disorders increases in proportion to the age of the study population. Verhulst et al. (1997) produced an estimate of the prevalence of psychiatric disorders in Dutch adolescents aged between 13 and 19. For the first screening, these researchers used the Child Behavior Checklist (CBCL; Aachenbach, 1991). The Diagnostic Interview Schedule for Children (DISC, child and parent version; Verhulst et al., 1997) was then used to determine the DSM-III-R diagnoses of the selected subjects $(N=780)$. The prevalence of anxiety disorders measured over a period of 6 months was more than $21 \%$. Anxiety disorders thus can be viewed as one of the most common psychiatric diagnoses in children and adolescents (Craske, 1997). 
It has been shown that the symptoms of children and adolescents with anxiety disorders are very likely to remain stable over time (Pollack, Otto, Rosenbaum et al., 1992). However, clinical observations suggest that the severity of the symptoms and the specific type of anxiety may change over time (Craske, 1997). In a prospective study, Last et al. (1996) found that the majority of children diagnosed with an anxiety disorder at intake were free of this diagnosis at the follow-up (three to four years later). However, these children did run a greater risk of being diagnosed with another psychiatric disorder, particularly another anxiety disorder. These findings suggest that it is not the anxiety disorders themselves that persist, but the factors underlying these anxiety disorders. Behavioral inhibition appears to be one of these factors.

Biederman, Rosenbaum, Hirschfeld et al. (1990) reported that the risk of developing multiple anxiety disorders in a clinical and non-clinical group is greatest for behaviorally inhibited children. Biederman, Rosenbaum, Bolduc-Murphy, and Faraone (1993) also found that young children who were classified as behaviorally inhibited at the start of the study were significantly more likely to have developed an anxiety disorder at the follow-up three years later than children who were not initially classified as behaviorally inhibited. It should be noted that particularly children with stable behavioral inhibition ran an increased risk.

Caspi et al. (1995) investigated the relationship between character traits at a very young age and the development of anxiety-related problems later in life. In their study, these researchers followed the development of 800 children over a period of 12 years (from age 3 to age 15). The results suggested a specific predictive value of temperamental traits related to behavioral inhibition. For instance, they found that boys who were inclined to explore new situations at a young age were less anxious in adolescence. In contrast, shy, avoidant and passive toddlers appeared to be more anxious at a later age.

Muris et al. (1999) examined the relationship between self-reported levels of behavioral inhibition and psychopathological symptoms. This investigation differed from the abovementioned studies in that the study population was comprised of adolescents (12 to 14 years of age, $N=152$ ) and the survey method involved self-reporting questionnaires. The researchers found that children who reported a high level of behavioral inhibition had higher scores on anxiety and depression scales than children who reported average or low levels of behavioral inhibition. These results were later replicated in a follow-up study among a larger group of adolescents $(N=968)$ with a wider age range (age 12 to 18; see Muris, Merckelbach, Schmidt, Gadet, \& Bogie, 2001). In the latter study, the possible 
relationships between behavioral inhibition, anxiety and depression were examined in more detail using structural equation modeling. It turned out that the data were best described by a model in which behavioral inhibition resulted in anxiety, and anxiety in turn led to depression. These findings match the results of a longitudinal study by Cole, Peeke, Martin, Truglio, and Seroczynski (1998) who also found that a high level of anxiety symptoms in children was of predictive value for the severity of depressive symptoms a few years later.

Although the abovementioned studies suggest that behavioral inhibition is associated with the development of a wide range of anxiety-related symptoms and disorders, there are also several studies that suggest that behavioral inhibition is a more specific risk factor. Townsley-Stemberger, Turner, Beidel, and Calhoun (1995), for instance, found that patients with specific or generalized social phobia reported significantly higher levels of shyness (a form of social inhibition) in childhood than controls. In line with these findings, Mick and Telch (1998) asked students with high scores on social or generalized anxiety scales to retrospectively report their level of behavioral inhibition during childhood. These researchers found that behavioral inhibition in childhood was strongly associated with social anxiety in adulthood, but not with generalized anxiety. Hayward, Killen, Kraemer, and Taylor (1998) showed that students ( $N=2242$ ) who reported retrospectively that they were behaviorally inhibited during childhood were four to five times more likely to suffer from a social phobia in adolescence than students who reported that they were not behaviorally inhibited during childhood.

Numerous studies therefore support the relationship between behavioral inhibition and the development of anxiety disorders. The question is: through which factors is this relationship created? For a start, the previously mentioned genetic component may be involved. For instance, research has shown that children of parents with one or more anxiety disorders are more likely to develop anxiety disorders themselves. Biederman, Rosenbaum, Bolduc et al. (1991) found that children of parents with panic disorder and/or agoraphobia were more likely to develop anxiety disorders themselves.

Furthermore, Rosenbaum, Biederman, Gersen et al. (1988) reported that a large number of children of parents with panic disorders (with or without agoraphobia) are behaviorally inhibited. Rosenbaum, Biederman, Hirschfeld et al. (1991) and Rosenbaum, Biederman, Bolduc-Murphy et al. (1993) also found that parents of inhibited children ran a greater risk of developing an anxiety disorder. Results obtained by Cooper and Eke (1999) suggest a specific link between extreme shyness in 4-year-old children and a history of maternal social phobia. This could 
indicate that the anxiety disorder of the mother and the shyness of the child share a genetic basis. Because gene-environment interactions already shape human behavior during early development already, (e.g., Fox, Nichols, Henderson et al., 2005), this is not an issue of one simple gene, but more a case of complex geneenvironment interactions at the gene-allele level.

The question then arises whether children with a vulnerable temperament (behavioral inhibition) who have parents who suffer from an anxiety disorder run a greater risk of developing anxiety disorders than children with behavioral inhibition who have parents who do not suffer from an anxiety disorder. To investigate this question, Rosenbaum, Biederman, Bolduc et al. (1992) made use of the cohorts from the studies by Kagan et al. (1988) and Biederman et al. (1990). These children were tested for behavioral inhibition and the mothers were interviewed to determine whether the children suffered from any anxiety disorders. Based on the data obtained, the children were divided into three groups: (1) children with behavioral inhibition and an anxiety disorder, (2) children with behavioral inhibition, but without an anxiety disorder, and (3) children without behavioral inhibition and without an anxiety disorder. The parents were also interviewed to establish whether they suffered from anxiety disorders. The results showed that the parents of children with behavioral inhibition and an anxiety disorder suffered or had suffered from significantly higher levels of anxiety disorders than the parents of children from the other two groups. This seems to indicate that inhibited children with 'anxious' parents indeed run a greater risk of developing an anxiety disorder.

In summary, we can conclude that the relationship between behavioral inhibition and the development of anxiety disorders is evident. Studies show that the presence of anxiety disorders in the parents seems to play a part in this relationship. In all probability, the parents genetically transfer their 'anxious' vulnerability to their child. At the behavioral level, this vulnerability is expressed in behavioral inhibition. Behavioral inhibition would then be a risk factor for the development of anxiety disorders. But, if the parents have no anxiety disorder themselves, it seems that the chance that behavioral inhibition develops into an anxiety disorder is much lower than when one or both parents suffer from an anxiety disorder.

However, a number of other possibilities cannot be excluded at this point. One of those possibilities is that behavioral inhibition is simply another manifestation of anxiety, which can be identified at a younger age. Behavioral inhibition would then be a milder form of psychopathology, instead of a risk factor or predictor of psychopathology (Biederman et al., 1995). With regard to the relationship between behavioral inhibition and social anxiety, Turner et al. (1996) 
suggest that behavioral inhibition in childhood may lead to the poor development of social skills, so that the number of social situations in which the child fails increases, resulting in increased social withdrawal and avoidance. According to these researchers, behavioral inhibition could also be an expression of a more complex personality that is associated with the development of anxiety. It should also be noted that many of the quoted studies on behavioral inhibition as a specific risk factor only used retrospective reporting as a research method. When subjects report retrospectively on the level of their own behavioral inhibition in the past, the result may be distorted due to the presence of psychopathology (e.g., social anxiety) at the time of reporting. Distortion may also be the result of selective attention, a selective memory, or a prejudiced interpretation (Bögels, Van Oosten, Muris, \& Smulders, 2001).

\section{INTERACTION BETWEEN BEHAVIORAL INHIBITION AND OTHER RISK FACTORS}

Behavioral inhibition appears to be an important risk factor for the development of anxiety disorders, but not every child with an inhibited temperament develops an anxiety disorder and not every anxious child is behaviorally inhibited (see for instance Biederman et al., 1990; Turner et al., 1996). The question then arises under what circumstances vulnerable (inhibited) children develop an anxiety disorder, or what factors protect such children from developing an anxiety disorder. Such factors could include mother-child attachment and parenting style. Several animal studies of behaviorally inhibited monkeys demonstrate the influence of these factors; these studies are discussed in some detail below. This chapter will then address the interaction between behavioral inhibition and other risk factors for the development of anxiety disorders. In the next section, an attempt is made to unite all the risk factors into an integrated model.

Suomi (1997) has identified two subgroups of primates who display anomalous behavioral patterns that could result in an increased risk of pathology or even death. The behavior and physiological arousal pattern of one of these two subgroups is very similar to the behavior and physiological arousal pattern of the 10 to $15 \%$ of children who are behaviorally inhibited (also see Kagan, 1989). The animals in this subgroup (approximately $20 \%$ of the study population) can best be described as hyperreactive. Suomi (1997) provides an overview of a number of recent studies, including studies of this subgroup of primates. Suomi's overview indicates that (systematically manipulated) inadequate social attachment experiences 
at a young age (e.g. deprivation of maternal care) have long-term consequences at the behavioral and physiological level. The question now is whether this also works the other way round, i.e. whether high-quality attachment experiences at a young age result in behavioral and physiological improvement in temperamentally vulnerable primates. Dramatic differences were indeed observed in hyperreactive primates as a result of the temperament of the adoptive mother assigned to them. Hyperreactive primates who were adopted by an uncaring mother displayed the expected anomalies in exploration patterns and biological responses to minor disturbances in their environment. Hyperreactive primates who were adopted and raised by caring mothers, on the other hand, explored their environment more, displayed fewer behavioral disturbances and exhibited evolved behavior early in life (behavior that would normally not appear until later in life). These differences were also apparent when comparing the hyperreactive group with 'normal' primates. Notably, the attachment between the hyperreactive primates and their caring adoptive mothers seemed 'secure'. Furthermore, female primates in this subgroup continued the parenting style of their adoptive mother when they became mothers themselves (Suomi, 1997).

Fox and Calkins (1993) examined the influence of the interaction between the character traits of the child and the quality of attachment on the development of behavioral inhibition. They posed the question which temperamental factors of the child (at the behavioral and physiological level) are predictive of behavioral inhibition, which types of mother-child attachment would contribute to this prediction, and whether the quality of the attachment depended on the temperament of the child. The results suggest that behavioral inhibition is a function of the interaction between temperament and attachment classification, whereby the attachment classification in turn depends on the temperament of the child. The study by Fox and Calkins draws a distinction between 'secure' and 'insecure' attachment, whereby 'insecure attachment' is further subdivided into 'avoidant insecure attachment' and 'ambivalent insecure attachment'. Avoidant insecurely attached children scored significantly lower on the behavioral inhibition index (i.e., appeared less inhibited) than ambivalent insecurely attached children. Securely attached children did not display any characteristics of behavioral inhibition at age 2 .

The results obtained by Fox and Calkins (1993) show that the quality of the mother-child attachment is influenced by the child's temperament. Rubin, Nelson, Hastings and Asendorpf (1999) examined whether an inhibited temperament affects parents' rearing style. These researchers examined a specific parenting dimension, namely 'encouragement of independence'. This was measured by means of a 
questionnaire (examples of items: 'I let my child make his or her own choices', 'I encourage my child to be curious, to explore, and to ask questions'). The child's level of inhibition was determined using the previously mentioned behavioral observation procedure also used by Garcia Coll et al. (1984). The results indicated that shy, withdrawn children are raised by parents who find it very difficult to encourage their children to explore new situations. As a result, the avoidant behavior of the child is maintained or even becomes worse. It is important to note in this context that the parenting style is partly influenced by the child's inhibition and not the other way round (Rubin et al., 1999). Rubin et al. (1997) had previously established that children who are consistently inhibited (i.e., children who display inhibition in both social and non-social situations) have mothers who are very loving, overprotective, and extremely involved in their child's activities. At the same time, however, these mothers do not respond adequately to the signals given by their child and are not sufficiently sensitive to the child's needs. As a result, the child does not acquire an active coping style, remains extremely wary, develops no self-confidence or independence, and also thinks that it is very normal to be anxious and withdrawn in new situations.

The results obtained by Manassis et al. (1995) largely correspond to those obtained by Fox and Calkins (1993). Researchers had found previously that insecure attachment could be a risk factor for the development of anxiety disorders in childhood (Manassis, Bradley, Goldberg, Hood, \& Swinson, 1994; Warren, Huston, Egeland, \& Sroufe, 1997; see for an overview Bernstein, Borchardt, \& Perwien, 1996). The objective of later studies (e.g., Manassis et al., 1995) was therefore to determine whether inhibition and/or insecure attachment could be associated with early indications of anxiety disorders in a study population with an increased risk (i.e., young children of mothers with an anxiety disorder). No relationship was found between behavioral inhibition and the quality of attachment: that is, insecurely attached children did not run an increased risk of inhibition and vice versa. The results did show, however, that within the group of behaviorally inhibited children, insecurely attached children had the highest levels of anxiety symptoms. It appears that secure attachment acts as a protective factor for groups with an increased risk for the development of anxiety disorders. Note that Suomi (1997) had reached the same conclusion in an overview of primate studies.

Asendorpf (1993) views inhibited behavior as the end result of two different inhibition processes that are elicited by the unfamiliarity of the interaction partner and the anticipation of a negative social evaluation (e.g., being ignored). According to Asendorpf, individual differences in behavioral inhibition cannot be explained 
completely by temperament alone. The way in which children handle new situations and new people ('coping') also plays a role. In short, the character trait of 'inhibition' produces the same behavioral effect in all children during the first few minutes of an interaction with an unfamiliar individual. However, at a later stage in the interaction, the way in which children handle their inhibition (the type of 'coping strategy' that they choose) becomes more important as a predictor of their behavior than the initial level of their behavioral inhibition (Asendorpf, 1993). Asendorpf (1991) had previously found a positive correlation between failed social interactions and behavioral inhibition. An increase in the number of failed social interactions also entailed an increase in the level of behavioral inhibition. It appears that behavioral inhibition can be reinforced by negative social experiences.

Research has shown that the temperament of a child interacts to a large extent with the child's environment. It is known that the quality of the mother-child attachment depends on the sensitivity and responsiveness of the mother. It now appears that this mother-child relationship also partly depends on the temperament of the child. Inhibited behavior has been shown to be a function of the interaction between temperament and attachment classification. In addition, an inhibited child partly influences the rearing style of the parents. Moreover, the parenting style influences the way in which children handle their anxiety and extreme shyness ('coping'). An overprotective parenting style may cause the anxious and withdrawn behavior of the child to be preserved.

\section{INTEGRATED MODEL OF THE VARIOUS RISK FACTORS}

Several risk factors for the development of an anxiety disorder have been discussed above. Research shows that these factors are not independent, but interact with each other. To clarify this interaction, an attempt was made to construct a model showing the interrelationship between all these factors (Figure 1). The starting point in this model is a genetic biological vulnerability to sympathetic arousal. These innate physiological characteristics may be expressed at the behavioral level in the form of behavioral inhibition. Behaviorally inhibited children will avoid new, unfamiliar situations. Because they avoid these situations, they have fewer opportunities to acquire social skills and coping strategies. Research shows that the behaviorally inhibited temperament of the child and the parenting style of the parents influence each other. Shy, withdrawn children are raised by parents who find it very difficult to encourage their children to explore new situations. Consequently, the avoidant 
behavior of the child is maintained or even becomes worse. As a result, the child does not acquire an active coping style, remains extremely wary, develops no self-confidence or independence, and also thinks that it is very normal to be anxious and withdrawn in new situations. The quality of the mother-child attachment also influences the child's inhibited temperament and vice versa. Furthermore, specific learning experiences and environmental stressors can cause a vulnerable, inhibited

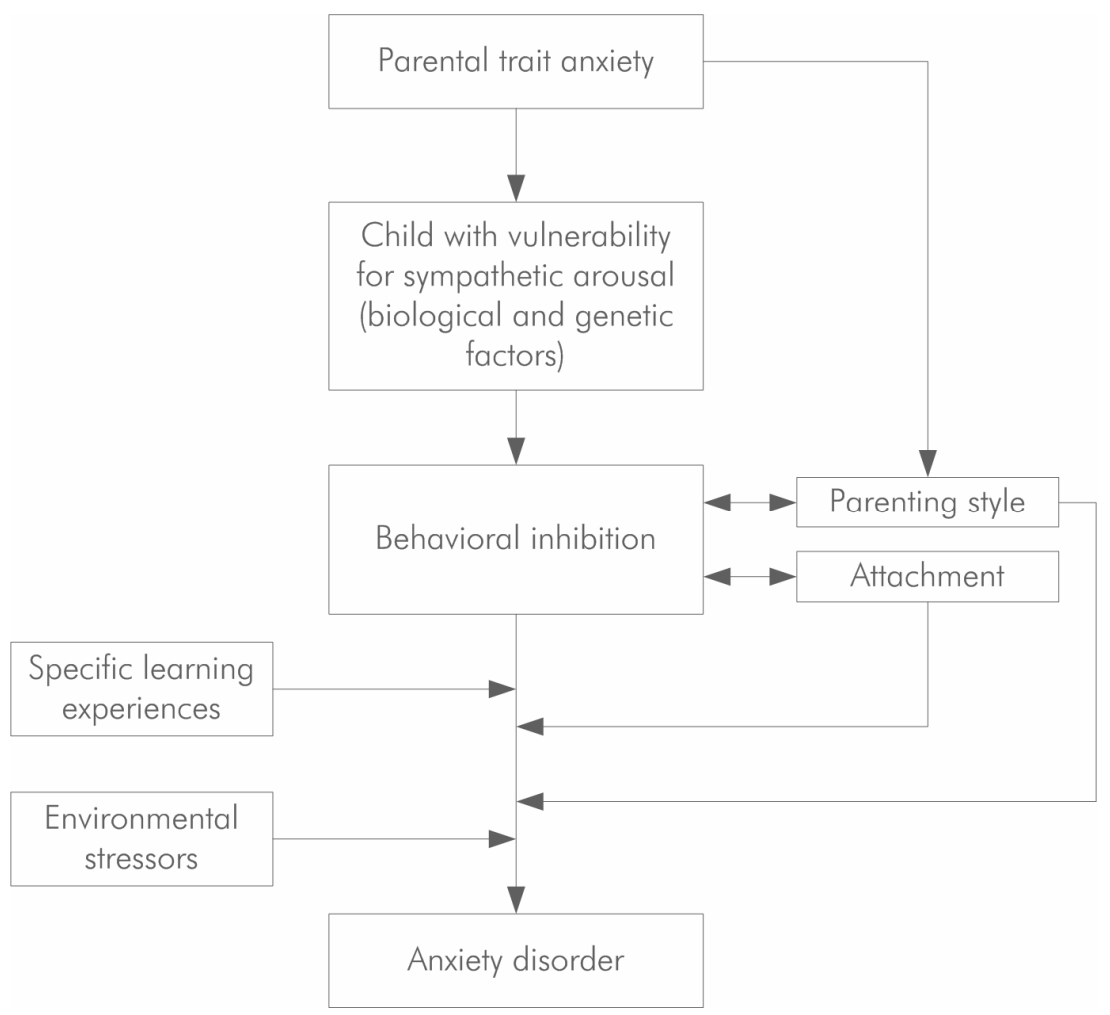

Figure 1. The role of behavioral inhibition and other risk factors in the development of childhood anxiety disorders 
child to develop an anxiety disorder later in life. The model includes parenting style and quality of attachment as risk factors that may contribute to the development of an anxiety disorder. It has been shown that these factors act not only as risk factors, but also as protective factors for behaviorally inhibited children. In other words, the risk that inhibited children will develop anxiety disorders diminishes when their parents encourage them to explore new situations.

\section{BEHAVIORAL INHIBITION AND ANXIETY: TAUTOLOGICAL?}

Another issue which needs attention is the possible tautological nature of the relationship between behavioral inhibition and anxiety. As mentioned in the introduction of this chapter, the construct of behavioral inhibition by definition already shows some overlap with the diagnostic criteria of anxiety disorders. For instance, 'withdrawal behavior in new, unfamiliar situations' can be viewed as avoidance behavior, which of course is an essential component of various anxiety problems. Probably the best way to distinguish between an inhibited temperament and anxiety disorder is to focus on interference with daily functioning (Hudson \& Rapee, 2004; Rapee, Kennedy, Ingram, Edwards, \& Sweeney, 2005; Rapee \& Spence, 2004). When a child is diagnosed, this by definition means that his/her symptoms cause significant interference in daily life. When classified as behavioral inhibited, a young person might show the same (avoidant) behaviors as a child with an anxiety disorder diagnosis, but the behavioral inhibited person does not really experience his/her inhibited characteristics as a problem in daily life (Rapee et al., 2005).

Moreover, research has demonstrated the difference between temperament and psychopathological symptoms. For example, Rapee and colleagues (2005) conducted an early prevention study in which high risk (i.e., behaviorally inhibited) children were randomly assigned to a 6-session parent-education program or no intervention. Results indicated that the intervention was more effective in reducing anxiety disorders as compared to no intervention. Most importantly, no significant intervention effects on children's temperament were found, which ofcourse points out that there is a difference between an inhibited temperament and anxiety disorders. In Rapee et al.'s words: "a direct effect of treatment on disorder was shown that was not mediated (as hypothesized) via change in temperament" (p.494). In a similar vein, Lemery, Essex, and Smider (2002) asked a group of experts to sort out items taken from behavioral rating scales for assessing temperament and psychopathology 
in youths. Results showed that most items were correctly assigned to either the temperament or the psychopathology construct. Furthermore, these researchers subjected longitudinal temperament and psychopathology data of a large sample of children to a factor analysis. This procedure clearly yielded a two-factor solution with most temperament items loading on one factor and most psychopathology items loading on the other factor. Interestingly, the relations between temperament and psychopathological symptoms remained significant after eliminating overlapping items, suggesting that this link was not due to measurement confounding (for similar results, see Van Brakel et al., 2004).

With regard to the difference between behavioral inhibition and social anxiety, it is important to keep the role of children's level of socio-cognitive maturity in mind. Social phobia can only be diagnosed as such when children are cognitively mature enough to make socially threatening evaluations of situations in which they have to interact with other people (Westenberg, Siebelink, \& Treffers, 2001). As such, social phobia most frequently has its onset in middle to late adolescence (see American Psychiatric Association, 2000). In contrast, behavioral inhibition already becomes manifest at a very early age (e.g., Garcia-Coll et al., 1984; Kagan et al., 1988). For example, when confronted with a new/unfamiliar toy or person 4-monthold infants show a combination of high motoractivity (moving arms and legs) in combination with negative affect (crying) as signs of the behaviorally inhibited temperament. On the basis of these early signs, it becomes possible to select young children and to implement prevention programs, in order to avert they will develop significant anxiety problems in later life. The latter point stresses the importance of the behavioral inhibition construct in relation to psychopathology.

In conclusion, although behavioral inhibition and anxiety clearly share certain features, there is sufficient evidence to consider them as related but distinct construct. 


\section{LIMITATIONS OF BEHAVIORAL INHIBITION RESEARCH}

Studies of behavioral inhibition have a number of limitations. In the first place, there is a problem of definition when we speak of 'social withdrawal', 'social inhibition', 'social isolation' and 'shyness'. Do these terms all have the same meaning, are they exchangeable, or is one concept perhaps covered by the meaning of another, broader concept? In the scientific literature, researchers often fail to indicate what principles they use to operationalize the concept of behavioral inhibition. Secondly, different terms are used to designate the group of non-inhibited children. One researcher may use 'not inhibited', the other 'uninhibited'. These two concepts are not fully identical. The 'inhibited-uninhibited' pairing refers to two extremes. This means that a comparison is made between children who score extremely high on the behavioral inhibition scale and children who score extremely low. In contrast, studies that draw a distinction between children who are 'inhibited' and those who are 'not inhibited' draw a comparison between children who score extremely high on the behavioral inhibition scale and children who display a normal profile. An additional problem is that children classified as 'uninhibited' (i.e., children with extremely low scores on the behavioral inhibition scale) may also display psychopathology, particularly behavioral disorders. Naturally, a group of children that may display psychopathology is not an ideal control group. Another point deserving attention is the stability of behavioral inhibition over time. Some researchers determine the level of inhibition at a particular point in time and then spend years investigating various correlates for this group of children. As indicated above, the stability of behavioral inhibition as a character trait is of particular importance in this type of research. Stability can be determined by measuring the level of behavioral inhibition at several points in time. If measurements are conducted only once, the study population is still heterogeneous, making it difficult to compare the results of different studies. The wide variety of study methods is also an important problem in behavioral inhibition research. The situations created to observe the child's behavior must be adjusted to the age of the subject. Several suggestions have already been given in this article. The importance of conducting such observations in a social and in a non-social context has been noted. Furthermore, the assessment of the child's parents and teachers should be taken into account in the behavioral inhibition index. The following variables are often used to measure behavioral inhibition: the latency period before playing with, talking to, or reacting to an unfamiliar person, and the period of time during which the child stays near the mother. Generally speaking, 
these variables are used in almost every study, but in widely varying observation scenarios. As a result, comparing different studies becomes virtually impossible. A standardized (public) protocol is therefore necessary, detailing the procedures used in behavioral observation, among other matters. The measurement of the physiological correlates of behavioral inhibition also tends to create problems, as the children are generally still quite young. The equipment used should preferably be non-invasive and applied in such a way that the children do not experience any nuisance, as the child's arousal as a result of the equipment may distort the results. For instance, measuring brain activity during behavioral observation is still virtually impossible.

\section{CONCLUSION}

Kagan and his study group state that 10 to $15 \%$ of all children are born with a genetically determined physiological predisposition ensuring that these children display a high level of motor activity as infants, cry in reaction to new stimuli, and are shy, withdrawn and anxious in new social and non-social situations when they grow up. This character trait seems relatively stable and appears to be a risk factor for the development of anxiety disorders. Some researchers have put forward the alternative hypothesis that behavioral inhibition is a milder form of psychopathology, instead of a risk factor or predictor of psychopathology. However, this does not set aside that various studies have demonstrated that this temperamental construct has considerable explanatory power for predicting the development of anxiety disorders in children.

Not every child with an inhibited temperament develops an anxiety disorder, and not every anxious child is behaviorally inhibited. It appears that environmental factors play an important moderating role. In the case of behavioral inhibition, the interaction between parent and child - particularly the parenting style, the quality of attachment, and the presence of psychopathology in the child's parents - could play an important role in determining whether or not the child will develop an anxiety disorder. Most probably, behavioral inhibition is one of the factors that increase a child's vulnerability for developing an anxiety disorder, as it has been demonstrated that behavioral inhibition is neither a necessary nor a sufficient cause of the development of anxiety disorders.

A number of limitations of behavioral inhibition studies have been noted. Despite these limitations, behavioral inhibition research has produced enough evidence to support an explanatory relationship between behavioral inhibition and 
the development of anxiety disorders in children. It should be clear that more extensive research is required, also in light of the fact that anxiety problems are generally persistent in nature and may lead to depression later in life, as suggested by several studies (e.g. Cole et al., 1998).

\section{PRESENT DISSERTATION}

Several risk factors for the development of an anxiety disorder have been discussed above. Available research seems to suggest that these factors do not operate in isolation, but interact with each other. To clarify this interaction, an attempt was made to construct a model showing the interrelationships among these factors. The model is shown in Figure 1 and has been discussed before in this chapter. The model proposes that in particular parenting styles, quality of attachment, and the presence of psychopathology in the child's parents are relevant in a context of a study on the role of behavioral inhibition in the etiology of childhood anxiety disorders. Taking the model as a point of departure, the research project that is described in this thesis was designed (1) to study the role of behavioral inhibition in the development of childhood anxiety disorders, (2) to examine the specificity of behavioral inhibition in predicting social anxiety as opposed to anxiety disorder symptomatology in general or even other types of psychopathology (e.g., depression), and (3) to investigate how behavioral inhibition interacts with other risk factors (i.e., insecure attachment, parental anxiety, negative rearing behaviors, and life events) in the pathogenesis of childhood anxiety problems. These questions were investigated in an experimental study (Chapter 4), in a cross-sectional study (Chapter 5), and in a longitudinal study (Chapter 6). Furthermore, Chapter 2 and 3 describe two psychometric studies in which the validity and reliability of the Behavioral Inhibition Scale was investigated.

More specifically, the study described in Chapter 2 examined the relationship between the Behavioral Inhibition Scale (BIS), a measure that was specifically developed for assessing behavioral inhibition, and behavioral observations of this trait. Moreover, the distinction between social and non-social behavioral inhibition gets further attention in this chapter. The study outlined in Chapter 3 investigated the test-retest stability of the BIS and the associations of this measure with the Early Adolescent Temperament Questionnaire-Revised (EATQ-R). The experimental study in Chapter 4 examined the startle reflex as a physiological marker of behavioral inhibition. The cross-sectional study in Chapter 5 was an attempt to examine the reciprocal connections among temperament, attachment, 
and parental rearing styles, and their unique and interactive relations to anxiety symptoms. The study described in Chapter 6 examined the additive and interactive effects of behavioral inhibition and a wide range of other vulnerability factors in the development of pathological anxiety in youths using a longitudinal research design. 



\section{CHAPTER 2}

Relations between parent-and teacherreported behavioral inhibition and behavioral observations of this temperamental trait 


\section{ABSTRACT}

Examined was the relationship between the Behavioral Inhibition Scale (BIS), a measure that was specifically developed for assessing behavioral inhibition, and behavioral observations of this trait. Children, aged 6-10 years, participated in a series of experimental tasks assessing behavioral features of the inhibited temperament. The parents and teachers of these children completed the BIS. Results showed that the BIS (in particular the parent version) was significantly related to the observational index of behavioral inhibition. An additional aim of the study was to examine the relationship between behavioral inhibition as indexed by the BIS and observations, on the one hand, and measures of anxiety symptoms, fear, and behavioral symptoms, on the other hand. As expected, significant correlations between behavioral inhibition indices and symptoms of anxiety and withdrawal were observed. The BIS is found to be a brief and easy-to-administer instrument, which seems to provide a meaningful first impression of children's level of behavioral inhibition and this is particularly true when using the parent version of the BIS. 


\section{RELATIONS BETWEEN PARENT- AND TEACHER- REPORTED BEHAVIORAL INHIBITION AND BEHAVIORAL OBSERVATIONS OF THIS TEMPERAMENTAL TRAIT}

Behavioral inhibition is a temperamental trait characterized by a relatively consistent pattern of behavioral and emotional responses to unfamiliar or novel people, situations or objects. Inhibited children typically respond to novel situations or objects with restraint, caution, and withdrawal, and with unfamiliar people, they are usually timid, fearful, and shy (Kagan, Reznick, \& Snidman, 1988). Research indicates that the temperamental construct of behavioral inhibition is fairly stable over time (Kagan et al., 1988; Scarpa, Raine, Venables, \& Mednick, 1995; Gest, 1997; for a review see Turner, Beidel, \& Wolff, 1996). Moreover, inhibited children seem to be at risk for developing anxiety disorders (e.g., Biederman, Rosenbaum, Hirshfeld et al., 1990; Biederman, Rosenbaum, Bolduc-Murphy et al., 1993; Muris, Merckelbach, Wessel, \& van de Ven, 1999; for reviews see Biederman, Rosenbaum, Chaloff, \& Kagan, 1995; Turner et al., 1996).

Longitudinal behavioral genetic analyses suggest that the relationship between behavioral inhibition (in particular, shyness) at 1, 11/2,2, and 3 years of age and later internalizing problems are largely due to genetic factors (Schmitz, Fulker, Plomin et al., 1999). Many studies support the hypothesis that behaviorally inhibited children are born with a genetically transmitted vulnerability for sympathetic arousal (e.g., Garcia Coll, Kagan, \& Reznick, 1984; Kagan et al., 1988; Snidman, 1989). That is, 10 to $15 \%$ of the children are born with a lower threshold for limbichypothalamic arousal to unexpected changes in the environment or novel events that cannot be assimilated easily (Kagan et al., 1988). As these children seem to be at risk for anxiety disorders, it may be relevant to develop instruments in order to detect behaviorally inhibited children before they become highly anxious. Early detection of these vulnerable children would make it possible to implement prevention programs. Such programs should focus on encouraging children to use problem-focused instead of avoidant and emotion-focused coping strategies as well as teaching parents to reduce anxious and overprotective rearing behaviors (Spence, 2001).

Most behavioral inhibition studies have employed extensive laboratory procedures to assess behavioral (e.g., proximity to the parent, latency to approach peers and adults) and physiological (e.g., heart rate variability, levels of secreted cortisol) features of the inhibited temperament. While these methods certainly provide 
valuable information about the behavioral inhibition construct, they also represent a rather time-consuming way for researchers who want to identify youth at risk for developing anxiety disorders. Inspired by the work of Reznick, Hegeman, Kaufman, and Woods (1992) and Gest (1997), Muris, Merckelbach, Wessel, and Van de Ven (1999) recently developed a self-report rating scale, the Behavioral Inhibition Scale (BIS). The BIS consists of items covering shyness (e.g., "I am shy when I have to talk to an unfamiliar child"), communication (e.g., "I talk easily to an unfamiliar child"), fearfulness (e.g., "I feel nervous when I have to talk to an unfamiliar child"), and smiling (e.g., "I feel good and I am able to laugh, when I talk to an unfamiliar child").

Three studies have tested the validity of the BIS. In the first study, Muris et al. (1999) administered the BIS to a group of nonclinic referred children $(N=152)$ aged 12 to 14 years. As expected, results showed that there were positive associations between behavioral inhibition as indexed by the BIS and symptoms of anxiety. These findings were replicated in a second study of a larger group of nonclinic referred adolescents $(N=968)$ with a broader age range (12 to 18 years; Muris, Merckelbach, Schmidt, Gadet, \& Bogie, 2001). Again, high BIS scores were accompanied by high levels of anxiety symptoms. In the third and final study, Muris, Meesters, and Spinder (2003) further examined the association between the BIS and anxiety in a sample of (nonclinic referred) young adolescents aged 11 to 15 years, not only relying on youths' self-report but also including the parents' point of view. Thus, children completed the BIS, while parents filled in a parent version of the measure. Results indicated that agreement between adolescents and their parents was moderate but significant $(r=.49)$. Furthermore, high levels of behavioral inhibition were associated with high levels of anxiety disorder symptoms, and this was true for both child and parent ratings.

Thus, behavioral inhibition in youth can be assessed by means of behavioral observations and questionnaires. Comparison of these methods may not only yield information about the internal structure of behavioral inhibition, but could also provide information on the degree to which parents, and eventually teachers, are able to rate children's level of inhibition. However, only a few studies have examined the relationship between an observational index of behavioral inhibition and questionnaires measures of behavioral inhibition.

Garcia Coll et al. (1984) compared behavioral observations of 21 -monthold children with two parental report indexes of behavioral inhibition. The first index was the Toddler Temperament Scale (TTS, Fullard, McDevitt, \& Carey, 1978), which was completed by both parents. The TTS is a 97 -item parent rating scale designed to 
assess a number of temperamental traits including approach-withdrawal, which bears strong resemblance to the construct of behavioral inhibition. The second index consisted of an interview with the mother who was asked to describe the child's current behavior and to recall how her child had responded to unfamiliar events during the first 2 years. The interview data were analyzed by an independent rater for the presence of inhibited behaviors. Garcia Coll et al. found positive correlations between the observational index of behavioral inhibition and scores on the approach-withdrawal scale of the TTS as obtained from fathers and mothers ( $r=.49$ and $r=.54$, respectively) and ratings of behavioral inhibition derived from the interview ( $r=.67$ ). Reznick, Gibbons, Johnson, and McDonough (1989) obtained laboratory observations of behavioral inhibition in a group of children at various ages $(14,20$, and 32 months) as well as parental ratings of inhibition-related traits as measured by TTS, the Infant Behavior Questionnaire (IBQ; Rothbart, 1981), the Infant Characteristics Questionnaire (ICQ; Bates, Freeland, \& Lounsbury, 1979), and the Emotionality-Activity-Sociability (EAS) Temperament Survey for Children (Buss \& Plomin, 1984). Results showed significant correlations between laboratory assessed behavioral inhibition and parental measures (with most rs in the .30-.40 range). Finally, Andersson (1999) obtained observations of behavioral inhibition in a sample of 2-year-old children as well as parental ratings of this temperamental construct using the EAS Temperament Survey for Children and the Emotional Expressions Questionnaire (EEQ; Hagekull \& Bohlin, 1995). Andersson reported a significant correlation between the observational behavioral inhibition index and a total rating scale score combining relevant scores of the EAS and EEQ $(r=.37)$.

Altogether, research indicates that observational indices of behavioral inhibition are moderately but significantly associated with parental reports of this construct. It is important to note, however, that previous studies have predominantly relied on existing temperamental rating scales for measuring behavioral inhibition from the parents' perspective. Although these scales tap temperamental dimensions (e.g., approach-withdrawal) which bear strong resemblance to behavioral inhibition, they were not developed specifically to assess behavioral inhibition. Given the importance of early detection of high-risk children, an economic and easy-toadminister scale for assessing behavioral inhibition in children would be very welcome. The current article describes a study which examined the validity of a rating scale, the BIS, that was specifically developed for assessing behavioral inhibition in children (Muris et al., 1999, 2001, 2003). The main purpose of the study was to investigate the relationship between the BIS and behavioral observations of this temperamental trait. For this purpose, a group of primary school children, aged 6- 
10 years, participated in a series of experimental tasks assessing behavioral features of the inhibited temperament. BIS data were obtained from parents and teachers. An additional aim of the study was to examine the relationship between behavioral inhibition as indexed by the BIS and observations, on the one hand, and measures of anxiety symptoms, fear, and behavioral symptoms, on the other hand.

\section{METHOD}

\section{Participants}

Participants were fifty-nine children $(29$ boys and 30 girls; mean age $=6.9$ years; $S D=0.8$, range 6-10 years') from two primary schools in Maastricht, The Netherlands, after obtaining written informed consent of the parents. Participation rate was $60 \%$, that is, 98 parents were approached to participate with their child and 59 parents finally agreed to participate. The percentages of families with a low, middle, or high socio-economic background (classified by means of the occupational levels of both parents, employing the guidelines provided by the Dutch Central Bureau of Statistics) were $45 \%, 40 \%$, and $15 \%$, respectively. The majority of the children and their parents were Caucasian (85\%). The remaining families (15\%) had a Mediterranean, Asian, or North-African background. About $90 \%$ of the children came from two-parent families.

\section{Procedure}

Parents were asked to complete parent versions of the BIS, the Screen for Child Anxiety Related Emotional Disorders (SCARED; Birmaher, Brent, Chiappetta et al., 1999), and the short version of the Social Competence and Behavior Evaluation (SCBE; LaFreniere \& Dumas, 1995). The teachers $(n=6)$ of the children completed the teacher version of the BIS.

Children first completed a questionnaire measuring fear symptoms (KFQ; Muris, Meesters, Mayer et al., 2003). This was done in their classrooms with the experimenter and teacher always being present to ensure confidential and independent responding and to provide assistance when necessary. After the classroom session, children were tested individually for approximately one hour in a separate room at school. Children participated in a series of behavioral tasks (see below). Children were videotaped during these tasks, so that it became possible to compute the interrater reliability for the various behavioral inhibition indices. 


\section{Questionnaires}

Parent and teacher versions of the BIS. The BIS version that was used in the present study was somewhat different from that employed in earlier research. While the BIS items of the previous version (e.g. see Muris et al., 1999) only referred to inhibited behaviors in relation to social situations, the version used in the present study distinguished between social and non-social inhibition, thereby acknowledging the fact that children's level of behavioral inhibition may vary considerably depending on the circumstances (see Reznick, 1989). Thus, the BIS version contained separate items for assessing inhibited behaviors during encounters with unfamiliar people (e.g., "My child is shy when he/she has to talk to an unfamiliar adult", "My child is shy when he/she has to talk to an unfamiliar child") as well as items referring to behavior in unfamiliar non-social situations (e.g., "My child plays easily with the displayed toys when we are in the waiting room of an unfamiliar doctor").

The teacher version of the BIS was identical to the parent version of this measure, except that items were rephrased in terms of the teacher's perspective (e.g., "This child is shy when he/she has to talk to an unfamiliar child"). Furthermore, the four items referring to unfamiliar non-social situations were reduced to two items and changed into unfamiliar non-social situations that were specific for the school setting (i.e., "This child is nervous when he/she has to change to new and unfamiliar study material" and "This child is nervous when he/she has to perform a new physical exercise. For example, when he/she has to climb a wall of bars for the first time"). The parent and the teacher version of the BIS were both rated on 4-point Likert scales with 1 = never, 2 = sometimes, $3=$ often, and $4=$ always. After recoding positive items, scores are summed to yield a BIS parent total score (range: 12-48), a BIS parent social score (range: 8-32), a BIS parent non-social score (range: 4-16), a BIS teacher total score (range: 10-40), a BIS teacher social score (range: 8-32), and a BIS teacher non-social score (range: 2-8).

The Screen for Child Anxiety Related Emotional Disorders (SCARED) is a questionnaire that attempts to measure childhood anxiety symptoms in terms of the Diagnostic and Statistical Manual of Mental Disorders (DSM-IV; American Psychiatric Association, 1994) taxonomy. In the present study, the 41-item parent version was used (Birmaher et al., 1999). Item examples are "My child worries about things working out for him" (generalized anxiety disorder), "My child doesn'† like being away from his family" (separation anxiety disorder), "When my child is frightened, his heart beats fast" (panic disorder). Parents have to indicate how frequently they think their child experiences each symptom on a 3 -point scale: 0 = almost never, $1=$ sometimes, and 2 often. A SCARED total anxiety score can be obtained by 
summing across relevant items. Previous research has demonstrated that the SCARED has good internal consistency (e.g., Muris, Schmidt, \& Merckelbach, 2000; Birmaher, Khetarpal, Brent et al., 1997), test-retest reliability, and discriminant validity (e.g., Birmaher et al., 1997). Furthermore, a recent study by Muris and colleagues (2004) has demonstrated that SCARED scores have considerable potential for predicting specific DSM-defined anxiety disorders in clinically referred children and adolescents.

The parent version of the Social Competence and Behavior Evaluation Scale (SCBE-30) is a 30-item questionnaire designed to assess patterns of anxiety/withdrawal, anger/aggression, and social competence (LaFreniere \& Dumas, 1995). Each of these factors consists of 10 items, e.g. "My child screams or yells easily" (anger/aggression), "My child remains apart, isolated from the group" (anxiety/withdrawal), and "My child cooperates with other children" (social competence). Items are scored on a 4 -point scale $(1=$ never, $2=$ sometimes, $3=$ often, and 4 =always). Previous research has shown that the SCBE-30 has good internal consistency with coefficients ranging from .77 to .92 and test-retest stability with correlations ranging from .61 to .86 (LaFreniere \& Dumas, 1996). Furthermore, a recent study demonstrated that the SCBE-30 discriminated between three groups of children with different behavioral problems (Kotler \& McMahon, 2002).

The Koala Fear Questionnaire (KFQ) was recently developed by Muris, Meesters, and Mayer (2000) as a standardized self-report instrument for assessing fears and fearfulness in children that can be used with children aged $4-12$ years. The scale consists of 31 items referring to potentially fear-provoking stimuli and situations (e.g., "Being hit by a car or truck", "Ghosts", "Spiders", "Heights", "Telling something in front of the class") that are all illustrated with pictures. Children rate the intensity of their fear of these stimuli and situations on a visual scale depicting Koala bears expressing various degrees of fear $(1=$ no fear, $2=$ some fear, $3=$ a lot of fear). A total score can be computed by summing the ratings across all items (range 31-93). Muris et al. (2003) examined the psychometric properties of the KFQ in six different studies and found that the KFQ is a reliable and valid scale for measuring fear in children as young as 4 years of age.

\section{Behavioral tasks}

The behavioral tasks used in this study were largely based on the procedures used by Kagan et al. (1988) who also exposed children in this age range to unfamiliar peers and adults and novel tasks and toys in order to obtain behavioral inhibition indices 
such as latency to approach, the amount of encouragement needed to perform a novel task, and lack of spontaneous talk.

Mystery guest. During this task, an unfamiliar person entered the room. This person was dressed up with a cape, a mask, and a wig. The child was instructed by the experimenter to 'unmask' this so-called mystery guest in order to check the identity of this person.

Cognitive test. The 'unmasked' mystery guest (a graduate research assistant) became the experimenter of the following task. The child was asked to sit down opposite the experimenter, on a small chair by a child-sized table. The experimenter then presented a cognitive-performance task. This task was taken from a Dutch intelligence test for children and asked the child to sort 6 cards into two categories (e.g., "flowers and fruits", "vehicles with a motor and vehicles without a motor"). The mean duration of this test was 8 minutes. During the test the experimenter tried to interact without words in order to elicit spontaneous verbal and non-verbal reactions from the child.

Play. Directly after the cognitive test the child was told he/she performed well, regardless of his/her real performance. Then the child was told to wait for a short period of time together with the research assistant in the same room. Meanwhile, the experimenter collected an unfamiliar peer who was randomly selected by the teacher from another class of the same school. This peer was unfamiliar to the child, 1 year older, and of the same sex as the child in question. Children were instructed to listen to a short fairytale told by the experimenter, and then to perform a play together based on the fairytale they just heard. The fairytale was a shortened version of "Little red riding hood and the big bad wolf". When children did not spontaneously start preparing the play, the experimenter invited them to do so after 3 minutes. Children were allowed to play as long as they liked. The mean duration of the play was 5 minutes.

Black boxes. After the play, the peer left the room and the child continued the experimental session with the "black boxes game". This game involved three boxes that were covered by a black cloth. The child was instructed to guess what objects were hidden in the boxes by touching the objects without seeing them. The child had to do this in a fixed order at his/her own tempo. Again, when the child did not spontaneously approach the boxes the experimenter invited him/her to do so (that is, 
when latency reached the maximum, see below). The first box was filled with a spiky broom, the second with a cuddly toy cat in a small bag that began to move and to scream upon touching it, and the third box contained sticky hair gel.

Computer game. The last task was a computer racing game. The child was instructed to drive as fast and as safe as possible to the end of the circuit. The circuit was situated in a mountainous surroundings with sharp bends, bad weather and bad sight. The child was not told that he/she could drive straight through all trees and rocks, he/she had to find out him/herself.

\section{Behavioral inhibition measures}

Spontaneous talk. As can be seen in Table 1, the child's amount of spontaneous talk was observed during each task. That is, during the mystery guest task, the cognitive performance task, the play, the black boxes game, and the computer racing game, the number of spontaneous utterances were counted. During the play, the child's spontaneous talk was divided into spontaneous talk to the peer and spontaneous talk to the experimenter.

Number of encouragements. The number of encouragements a child needed were observed during three tasks, viz. mystery guest, play, and black boxes. This measure was operationalized by counting the number of times the experimenter said, for example: "Go ahead!", or if it took the child some time to start with this task: "You can do this!". An encouragement was given when children did not proceed with the task for 1 minute.

Looking at experimenter. The number of times the child looked at the experimenter was counted during the mystery guest and the cognitive test.

Number of smiles. The child's showing of positive affect was rated by counting the number of smiles. This variable was obtained during the cognitive test, the play, the black boxes, and the computer game.

Number of proposals for play. In order to measure the child's tendency to take initiative, the number of proposals the child made for the play during the preparation phase were counted. 
BEHAVIORAL INHIBITION OBSERVATIONS

Table 1: Mean Scores (Standard Deviations) and Interrater Agreement for the Variables observed during the Behavioral Tasks and for the Questionnaire Scores

\begin{tabular}{|c|c|c|}
\hline Behavioral observation tasks & $M(S D)$ & $\begin{array}{l}\text { Interrater agreement } \\
\text { (kappa) }\end{array}$ \\
\hline \multicolumn{3}{|l|}{ Mystery Guest } \\
\hline $\begin{array}{l}\text { Spontaneous talk } \\
\text { Number of encouragements } \\
\text { Looking at experimenter } \\
\text { Latency for approaching }\end{array}$ & $\begin{array}{l}1.67(2.40) \\
1.62(1.24) \\
2.22(1.53) \\
0.09(0.29)\end{array}$ & $\begin{array}{l}.94 \\
.88 \\
.70 \\
-\end{array}$ \\
\hline \multicolumn{3}{|l|}{ Cognitive test } \\
\hline $\begin{array}{l}\text { Spontaneous talk } \\
\text { Looking at experimenter } \\
\text { Number of smiles }\end{array}$ & $\begin{array}{l}0.78(2.01) \\
8.45(4.54) \\
1.93(2.03)\end{array}$ & $\begin{array}{l}.93 \\
.91 \\
.88\end{array}$ \\
\hline \multicolumn{3}{|l|}{ Play } \\
\hline $\begin{array}{l}\text { Spontaneous talk to peer } \\
\text { Spontaneous talk to experimenter } \\
\text { Number of encouragements } \\
\text { Number of smiles } \\
\text { Number of proposals }\end{array}$ & $\begin{array}{l}1.08(1.42) \\
1.28(1.72) \\
2.32(1.83) \\
2.90(1.79) \\
0.70(1.03)\end{array}$ & $\begin{array}{l}.73 \\
.95 \\
.75 \\
.80 \\
.79\end{array}$ \\
\hline \multicolumn{3}{|l|}{ Black boxes } \\
\hline $\begin{array}{l}\text { Spontaneous talk } \\
\text { Number of encouragements } \\
\text { Number of smiles } \\
\text { Latency for approaching }\end{array}$ & $\begin{array}{l}1.32(1.36) \\
1.49(0.84) \\
2.63(1.66) \\
0.10(0.10)\end{array}$ & $\begin{array}{l}.86 \\
.68 \\
.85 \\
-\end{array}$ \\
\hline \multicolumn{3}{|l|}{ Computer game $^{a}$} \\
\hline $\begin{array}{l}\text { Spontaneous talk } \\
\text { Number of smiles }\end{array}$ & $\begin{array}{l}12.57(17.00) \\
4.64(6.49)\end{array}$ & - \\
\hline
\end{tabular}

Note. SCARED $=$ Screen for Child Anxiety Related Emotional Disorders, KFQ $=$ Koala Fear Questionnaire, SCBE = Sociale Competence and Behavior Evaluation Scale, BIS = Behavioral Inhibition Scale. ${ }^{\circ} T$ The computer game was not recorded on video. Observations were made online by only one observer so that interrater agreement could not be calculated. 
Table 1: Mean Scores (Standard Deviations) and Interrater Agreement for the Variables observed during the Behavioral Tasks and for the Questionnaire Scores (Continued)

\begin{tabular}{lll}
\hline Questionnaires & $M(S D)$ & Cronbach's $\alpha$ \\
\hline BIS parent & & \\
Total score & $24.19(5.98)$ & .86 \\
Social items & $16.60(4.56)$ & .89 \\
Non-social items & $7.60(2.13)$ & .46 \\
BIS- teacher & & .93 \\
Total score & $23.24(6.22)$ & .94 \\
Social items & $18.76(5.49)$ & .89 \\
Non-social items & $4.49(1.35)$ & .90 \\
SCARED & & .86 \\
Total score & $54.73(9.74)$ & .83 \\
Total score corrected for social & $34.34(6.20)$ & .89 \\
items & $20.29(4.53)$ & \\
Social items & $56.12(11.06)$ & .80 \\
KFQ & & .74 \\
SCBE & $17.42(3.71)$ & \\
Anger-aggression & $29.54(3.78)$ & \\
Social competence & $16.86(3.71)$ & \\
Anxiety-withdrawal & & \\
\hline Note. SCARED & & \\
\hline
\end{tabular}

Note. SCARED $=$ Screen for Child Anxiety Related Emotional Disorders, KFQ $=$ Koala Fear Questionnaire, SCBE = Sociale Competence and Behavior Evaluation Scale, BIS = Behavioral Inhibition Scale. ${ }^{a}$ The computer game was not recorded on video. Observations were made online by only one observer so that interrater agreement could not be calculated.

Latency for approaching mystery guest. Latency was measured in seconds: time started when the experimenter said: "Go ahead!" and stopped when the child started to take off the mystery guest's cape.

Latency for approaching boxes. Latency was measured in seconds: time started when the experimenter said: "Go ahead!" and stopped when the child moved his/her hand into the first black box. The child was instructed to do this task in a fixed order (that is, box 1, box 2, box 3) but on his/her own pace. Because of this, latency for the second box was measured from the moment the child pulled out his/her hand from the first box until the child moved his/her hand into the second box. Latency for the third box was measured from the moment the child pulled out his/her hand from the second box until the child moved his/her hand into the third box. When the task 
lasted 3 minutes (maximum time), the experimenter encouraged the child for the last time and then ended the task.

Composite behavioral inhibition index. The behavioral observations were video recorded. Two trained graduate research assistants coded the videotapes. For statistical analyses, all the measures were averaged across the different observers for each child. Then they were standardized and summed to yield a composite behavioral inhibition index. Several authors have noted that behavioral inhibition not only refers to negative features (e.g., shyness, withdrawal) but also to the absence of positive characteristics (e.g., smiling, approach; Andersson, 1999; Kagan, Snidman, Zentner, \& Peterson, 1999). For this reason, it is important that the composite index comprises negative and positive features. This was done in such a way that signs of negative affect contributed positively to the behavioral inhibition composite index and signs of positive affect (e.g. number of smiles) contributed negatively to the index. It should be noted that the latency measures were close to zero for most of the children, but they extended to many minutes (or the maximum) for the inhibited children. This asymmetric distribution was not usable for the composite inhibition index. Because latency seemed to be an important measure to include in the composite index, this variable was dichotomized so that inhibition was either present (i.e., latency of 1 minute or longer) or absent. After being dichotomized, this variable was standardized and included in the composite index.

\section{RESULTS}

\section{General findings}

Before addressing the main research questions, several preliminary analyses were conducted. First, as can be seen in Table 1, interrater reliabilities for behavioral inhibition variables were generally satisfactory, with kappas ranging between .68 and 95. Second, t-tests revealed that there were no significant gender differences on any of the measures. Third, questionnaires were reliable in terms of internal consistency. That is, most Cronbach's alphas were well above .70 for the parent and teacher versions of the BIS, the SCARED, the KFQ, and the various SCBE-30 scales (Table 1). The one exception was the non-social subscale of the BIS (alpha $=.46$ ). Fourth, correlations among observational measures of behavioral inhibition were generally low to moderate, but in the expected direction. For instance, the number of encouragements correlated positively with looking at the experimenter $(r=.27, p<$ $.01)$ and with latency $(r=.46, p<.01)$. Spontaneous talk was negatively associated 
with looking at the experimenter $(r=-.25, p<.05)$, but positively correlated with number of proposals for the play $(r=.23, p<.05)$. Taken together, correlations among various elements of the observational index varied between .01 and .46 , with a mean correlation of .22 $(p<.05)$. The internal consistency of the composite observational index was sufficient, with a Cronbach's alpha of .68.

Correspondence between child-, parent- and teacher-reported behavioral inhibition and the observational index

Correlations between questionnaire and observational measures of behavioral inhibition are shown in Table 2. Two conclusions can be drawn from this table. First, the observational index of behavioral inhibition was more substantially related to the parent version of the BIS than to the teacher version. Second, only BIS scores that pertained to parent- and teacher-reported behavioral inhibition in social situations were significantly linked to the observational composite.

Table 2 also displays correlations between the questionnaire data and specific aspects of observed behavioral inhibition. All significant relations were low to moderate, but in the expected direction. That is, parent-reported behavioral inhibition correlated positively with number of encouragements and latency (rs being .30 and .23), but negatively with spontaneous talk ( $r=-.31)$, number of smiles ( $r=$ -.27), and number of proposals for play $(r=-.22)$. Teacher-reported behavioral inhibition showed the same pattern, except for spontaneous talk and number of proposals for play, for which relations were non-significant.

\section{Relations between behavioral inhibition and anxiety/behavioral symptoms}

Parent-reported anxiety symptoms as indexed by the SCARED were significantly connected to parent-reported behavioral inhibition (rs between .42 and .62), teacher-reported behavioral inhibition ( $r$ s between .22 and .27), and the observational index $(r=.24)$. To control for shared variance due to item overlap and measurement confounding (Lemery, Essex, \& Smider, 2002), correlations were also computed between behavioral inhibition indices and corrected SCARED scores, from which social items were removed ${ }^{2}$. As can be seen in Table 3, this procedure resulted in an attenuation of the correlations between the BIS social items scores and parent-reported anxiety symptoms. However, the correlation between BIS parent social items and corrected SCARED remained significant $(r=.23)$. The correlation between BIS parent-non-social items and corrected SCARED was not affected by the procedure $(r=.56)$. 
Table 2 Correlations between Parent- and Teacher-reported Behavioral Inhibition, the Index derived from the Observations and the six Observational Tasks

\begin{tabular}{|c|c|c|c|c|c|c|c|}
\hline & $\begin{array}{c}\text { Bl Observational } \\
\text { index }\end{array}$ & $\begin{array}{l}\text { Spontaneous } \\
\text { talk }\end{array}$ & $\begin{array}{c}\text { Number of } \\
\text { encouragements }\end{array}$ & $\begin{array}{l}\text { Looking at } \\
\text { experimenter }\end{array}$ & Latency & $\begin{array}{c}\text { Number of } \\
\text { smiles }\end{array}$ & $\begin{array}{c}\text { Number of } \\
\text { proposals for } \\
\text { play }\end{array}$ \\
\hline \multicolumn{8}{|l|}{ BIS parent } \\
\hline Total score & $.47^{* *}$ & $-.37^{*}$ & $.30 *$ & .00 & $.23^{*}$ & $-.27^{*}$ & $-.22 *$ \\
\hline Social items & $.44^{* *}$ & $-.32^{* *}$ & $.33^{* *}$ & -.04 & $.27^{*}$ & $-.27^{*}$ & -.17 \\
\hline Non-social items & .73 & -.74 & .09 & -.02 & .02 & -.17 & $-.23^{*}$ \\
\hline \multicolumn{8}{|l|}{ BIS teacher } \\
\hline Total score & $.22^{*}$ & .07 & $.38^{* *}$ & -.10 & $.23^{*}$ & $-.27^{*}$ & -.19 \\
\hline Social items & $.24^{*}$ & .03 & $.37^{* *}$ & -.09 & .20 & $-.30^{*}$ & -.19 \\
\hline Non-social items & .02 & .20 & .17 & -.07 & .15 & .02 & -.04 \\
\hline
\end{tabular}

Note. BIS = Behavioral Inhibition Scale, BI Observational index $=$ Behavioral Inhibition composite index. $N=59 .{ }^{*} p<.05{ }^{* *} p<.01$ 
For teacher-reported behavioral inhibition, the association between nonsocial items and SCARED became stronger when deleting the social items in the SCARED $(r=36)$, whereas the previously significant correlation between BIS teacher-social items and SCARED disappeared. The significant correlation between the anxiety symptoms and the observational index of behavioral inhibition also disappeared after removing the social items from the SCARED. For the KFQ, only one significant correlation was observed with teacher-reported behavioral inhibition in non-social situations ( $r=.26)$; all other correlations were non-significant. Correlations between BIS scores and parent-reported behavioral symptoms of the child as indexed by the SCBE-30 yielded the expected results. As can be seen in Table 3, correlations between behavioral inhibition and anxiety/withdrawal were positive and significant (rs between .28 and .42), correlations with social competence were negative and significant ( $r$ s between -.21 and -.26), whereas correlations with anger/aggression were non-significant. A similar pattern of results was found for teacher-reported behavioral inhibition, although correlations were generally smaller.

Table 3: Correlations between Parent- and Teacher-reported Behavioral Inhibition and Parentreported Anxiety (SCARED) and Behavioral Symptoms (SCBE-30) and Child-reported Fear (KFQ)

\begin{tabular}{cccccc}
\hline SCARED & $\begin{array}{c}\text { SCARED } \\
\text { corrected for } \\
\text { social items }\end{array}$ & KFQ & AA & SC & AW \\
\hline
\end{tabular}

\begin{tabular}{|c|c|c|c|c|c|c|}
\hline \multicolumn{7}{|l|}{ BIS parent } \\
\hline $\begin{array}{l}\text { Total score } \\
\text { Social items } \\
\text { Non-social items }\end{array}$ & $\begin{array}{l}.57^{* *} \\
.42^{* *} \\
.62^{* *}\end{array}$ & $\begin{array}{l}.38^{* *} \\
.23^{*} \\
.56^{* *}\end{array}$ & $\begin{array}{l}.05 \\
.07 \\
-.02\end{array}$ & $\begin{array}{l}.06 \\
.02 \\
.17\end{array}$ & $\begin{array}{l}-.26^{*} \\
-.21^{*} \\
-.24^{*}\end{array}$ & $\begin{array}{l}.42^{* *} \\
.36^{* *} \\
.28^{*}\end{array}$ \\
\hline \multicolumn{7}{|l|}{ BIS teacher } \\
\hline $\begin{array}{l}\text { Total score } \\
\text { Social items } \\
\text { Non-social items }\end{array}$ & $\begin{array}{l}.26^{*} \\
.22^{*} \\
.27^{*}\end{array}$ & $\begin{array}{l}.17 \\
.10 \\
.36^{*}\end{array}$ & $\begin{array}{l}.18 \\
.74 \\
.26^{*}\end{array}$ & $\begin{array}{l}-.13 \\
-.15 \\
.06\end{array}$ & $\begin{array}{l}-.07 \\
-.06 \\
-.11\end{array}$ & $\begin{array}{l}.28^{*} \\
.23^{*} \\
.28^{*}\end{array}$ \\
\hline $\begin{array}{l}\text { BI Observational } \\
\text { index }\end{array}$ & $.24^{*}$ & .74 & .07 & .15 & -.05 & $.23^{*}$ \\
\hline \multicolumn{7}{|c|}{$\begin{array}{l}\text { Note. } N=59 . \text { SCARED }=\text { Screen for Child Anxiety Related Emotional Disorders (parent report) } \\
K F Q=\text { Koala Fear Questionnaire (child report), SCBE-30 = Social Competence and Behaviorc } \\
\text { Evaluation (parent report), } A A=\text { Anger/Aggression subscale, } S C=\text { Social Competenc } \\
\text { subscale, } A W=\text { Anxiety Withdrawal subscale, Bl Observational index = Behavioral Inhibitio } \\
\text { composite index. }{ }^{*} p<.05,{ }^{* *} p<.01 \text {. }\end{array}$} \\
\hline
\end{tabular}


The results described above indicate that parent- and teacher-reported BIS scores are correlated in a theoretically meaningful way to symptoms of anxiety (SCARED), withdrawal, social competence, and anger/aggression (SCBE-30). In order to further examine the convergent and divergent validity of the BIS, tests for comparing correlated correlations coefficients (Meng, Rosenthal, \& Rubin, 1992) were carried out. This analysis yielded mixed results (see Table 4). For example, results showed that the correlation between the BIS parent-total score and the observational index was not stronger than the correlation between the BIS parenttotal score and the SCARED-corrected for social items $(Z=0.19, p=.42)$. However, the correlation between the observational index and the BIS parent-total score was significantly stronger than the association between the observational index and the corrected SCARED $(Z=1.92, p<.05)$. Furthermore, in contrast to what was expected, BIS parent non-social scores correlated more substantially with the corrected SCARED than with the behavioral inhibition observational index. Finally, the correlation between the BIS total score and the observational index remained significant when controlling for corrected SCARED scores, $r=.39, p<.01$, whereas the correlation between the observational index and corrected SCARED disappeared when holding BIS scores constant $(r=.00)$.

Table 4: Correlation Coefficients examining the Convergent and Divergent Validity of the BIS

\begin{tabular}{lll}
\hline & Bl observational index & $\begin{array}{c}\text { SCARED corrected for social } \\
\text { items }\end{array}$ \\
\hline $\begin{array}{l}\text { SCARED corrected for } \\
\text { social items }\end{array}$ & $.14_{a}$ & \\
BIS Parent & & \\
Total score & $.47_{b}$ & $.38_{b}$ \\
Social score & $.44_{b}$ & $.23_{b}$ \\
Non-social score & $.13_{a}$ & $.56_{b}$ \\
\hline
\end{tabular}

Note. $N=59 . B I S=$ Behavioral Inhibition Scale, Bl observational index = Behavioral Inhibition composite index, SCARED = Screen for Child Anxiety Related Emotional Disorders. Correlations within the left column and correlations between the left and right column (within the same row) which do not share similar subscripts, differ at $p<.05$.

\section{DISCUSSION}

Previous research indicates that observational indices of behavioral inhibition are moderately, but significantly, associated with parental reports of this construct or 
related phenomena (e.g., Andersson, 1999; Garcia Coll et al., 1984; Reznick et al., 1989). The main purpose of the present study was to investigate the relationship between the BIS, a measure that was specifically developed for assessing behavioral inhibition, and behavioral observations of this temperamental trait. Moderate but significant relations were found between parent- and teacher-reported behavioral inhibition of the child as measured by the BIS and the observational index of this specific behavioral pattern, although parental ratings were more convincingly related to children's actual behavior in unfamiliar situations than teacher ratings. Furthermore, only social items of the BIS (i.e., items referring to encounters with unfamiliar people) were associated with the observational indices of behavioral inhibition.

Although significant, the relations between parent-reported behavioral inhibition and the observational indices were moderate. Epstein $(1979,1980)$ noted that in the case of observational assessment of temperamental traits, sampling error is large when behavior is measured only once. Epstein clearly demonstrated that correlations between traits and actual behavior become stronger when the pertinent behavior is measured in multiple situations for which scores are combined. Although the present study included various behavioral tasks, it is possible that the number of different situations was still too small to reliably assess inhibited temperament. Also, social items of the BIS were more strongly associated with the observational index of behavioral inhibition than non-social items. It may well be the case that the observational tasks that were employed in this study were more suitable for assessing social aspects than non-social aspects of behavioral inhibition. For example, while the black boxes were intended as a non-social task, the child was still confronted with an unfamiliar adult (i.e., the experimenter) and this may have given the task a more social character. Thus, the child's behavior during this task may have been more determined by the presence of the unfamiliar adult than by the exploration of the unfamiliar objects in the black boxes. Finally, correlations between teacher-reported behavioral inhibition and the observational index were rather low. These results are in line with previous research, indicating that children's internalizing problems remain largely hidden at school (Muris \& Meesters, 2002).

Parent-reported anxiety symptoms as indexed by the SCARED were moderately associated to behavioral inhibition as measured by the BIS, a result that is in line with a previous study by Muris et al. (2003). Because item overlap might account for this relationship (Lemery et al., 2002), correlations between the BIS and the SCARED were also computed after removing social items from the latter instrument. The results showed that the associations between parent-reported behavioral inhibition 
and the SCARED attenuated as a result of this procedure. Nevertheless, most correlations between BIS and SCARED remained statistically significant. The association between the parent version of the BIS and child report of fear (KFQ) was not significant. While this result is not in keeping with our expectations, this link involved a cross-informant correlation between two related but different constructs (i.e., behavioral inhibition and fear; e.g., Murphy \& Davidshofer, 1994).

The convergent and divergent validity for the BIS was further examined in various ways. First, its relations with parent-reported behavioral symptoms of the child as indexed by the SCBE-30 were explored. As predicted, results showed that parent-reported behavioral inhibition was positively associated with anxiety/withdrawal and, at least to some extent, negatively linked to social competence. No association was found between the BIS and the SCBE-30 subscale of anger/aggression. Second, tests for correlated correlations coefficients were used to compare the correlations among the BIS, the observational index, and the SCARED (corrected for social items). To some extent, results showed the predicted pattern. That is, the observation index of behavioral inhibition was more strongly associated with the parent version of the BIS than with anxiety symptoms. Third, the correlation between the BIS and the observational index did not disappear when controlling for anxiety, thereby indicating that the correlation between the two behavioral inhibition indices was not mainly carried by their shared variance with anxiety symptoms. Altogether, these data provide support for the validity of the BIS.

Results showed that correlations among observational measures were moderate. Moreover, an additional analysis revealed that only number of proposals for the play and latency were substantially related to the composite behavioral inhibition index. Consequently, one could argue that the other measures were less sensitive indicators of inhibited behavior. However, the present study relied on a relatively small sample of non-clinical children. Because only 10 to $15 \%$ of children clearly shows the signs of behavioral inhibition (Kagan et al., 1988), it is plausible to assume that only few children in the present sample were extremely inhibited and this may have hindered the finding of more substantial associations among various observational indexes.

Besides the fact that our study only included a small number of participants, several other limitations should be acknowledged. First, as mentioned earlier, the present study was cross-sectional in nature. Longitudinal studies would provide important additional information on the value of questionnaire-rated behavioral inhibition in predicting inhibited behaviors, and in its wake, anxiety symptoms (cf. Biederman et al., 1993). Second, a methodological drawback of the present study 
was that six teachers completed the BIS for the 59 participating children. Although it was not possible to correct for this non-independence of assessment due to the small sample size, a preliminary analysis did not reveal significant differences among the ratings of the teachers, suggesting that there was no systematic bias. Third, for some of the behavioral inhibition variables, it would have been preferable if the durations of various tasks for individual children would have been taken into account. For instance, in the case of spontaneous talk, it is possible that differences in time to finish the task affected the number of utterances, thereby influencing the observed level of behavioral inhibition with this variable.

Taken together, the present data showed that there were moderate but significant relations between parent- and teacher-reported behavioral inhibition as indexed by the BIS and behavioral observations of this temperamental trait. Further, the BIS correlated in a theoretically meaningful way with indices of psychopathology (i.e., fear, anxiety, anger/aggression). Thus, the BIS is a brief and easy-to-administer instrument, which seems to provide a meaningful first impression of children's level of behavioral inhibition and this is particularly true when using the parent version of the BIS. Future studies should examine whether the BIS has any incremental value as an index of behavioral inhibition above previously employed subscales of temperamental questionnaires. Moreover, the items of the non-social subscale require improvement so that they become more sensitive for measuring behavioral inhibition, and hence show better convergent validity with observations of this temperament trait. A careful study of the non-social elements of behavioral inhibition in children of varying ages seems necessary to achieve this goal, as it seems to be the case that nonsocial behavioral inhibition might be even more prone to developmental changes than social behavioral inhibition (see for a discussion: Van Brakel, Muris \& Bögels, 2001). 




\section{CHAPTER 3}

A brief scale for measuring "behavioral inhibition to the unfamiliar" in children 


\section{ABSTRACT}

The Behavioral Inhibition Scale (BIS) is a brief rating scale for measuring behavioral inhibition in children. The present study examined the test-retest stability of the BIS in a sample of 7 to 12 year-olds consisting of inhibited and non-inhibited children ( $N=$ 83). Results demonstrated that the BIS scores were fairly stable over a 2-year period, with a test-retest correlation of .77. Further, BIS scores of inhibited children significantly increased over the 2-year period, whereas those of the non-inhibited comparison group significantly decreased. Finally, the BIS was in a theoretically meaningful way associated with temperamental traits as measured by the Early Adolescent Temperament Questionnaire-Revised (EATQ-R; Ellis \& Rothbart, 2001). Altogether, these results provide support for the reliability and validity of the BIS. 


\section{A BRIEF SCALE FOR MEASURING "BEHAVIORAL INHIBITION TO THE UNFAMILIAR" IN CHILDREN.} "Behavioral inhibition to the unfamiliar" refers to a temperament-based individual
difference variable, which can be defined as "the tendency to exhibit fearfulness,
restraint, and withdrawal in the face of novel events or situations, including
unfamiliar rooms, toys, peers, an adults" (Hirshfeld-Becker, Biederman, \&
Rosenbaum, 2004, p.27; see also Kagan, 1994). Research has indicated that
children who are behaviorally inhibited seem to be at increased risk for developing
anxiety disorders. Noteworthy in this regard is a longitudinal study by Biederman and
colleagues (1990, 1993). In that study, pre-school children were followed for a 3-
year period. Results showed that children initially identified as behaviorally inhibited
were subsequently more likely to develop anxiety disorders compared to control
children (i.e., children who at study onset were not classified as behaviorally
inhibited). Not only social phobia, but also separation anxiety disorder, and multiple
anxiety disorders were significantly more prevalent in the subsample of children with
behavioral inhibition (for a review of the research on the link between behavioral
inhibition and anxiety symptoms in youths, see Hirshfeld-Becker et al., 2004).

Behavioral inhibition is typically assessed by means of extensive laboratory procedures during which children are exposed to various types of novel social and non-social stimuli in order to assess behavioral (e.g., proximity to the parent, latency to approach novel stimuli and unfamiliar persons) and physiological (e.g., heart rate variability, cortisol levels) features of this temperamental trait. While these methods provide important and valuable information on children's level of behavioral inhibition, they also represent a rather time-consuming way for researchers and clinicians who want to identify youth at risk for developing anxiety disorders. Behavior rating scales might prove to be a good alternative method for measuring behavioral inhibition in children. Until recently, psychologists used existing temperamental rating scales (e.g., the Toddler Temperament Scale; Fullard, McDevitt, \& Carey, 1978) for this purpose, as these scales typically include dimensions such as approachwithdrawal that bear strong similarity with behavioral inhibition. In the past years, however, several attempts have been made to develop rating scales that specifically tap the characteristic features of behavioral inhibition. One example of such a scale is the Behavioral Inhibition Questionnaire (BIQ; Bishop, Spence, \& McDonald, 2003), which is a 30-item parent and teacher report scale, measuring children's inhibited behaviors in various domains: relationships with peers and adults, exposure to physically challenging activities and unfamiliar situations, and separation and 
performance situations. The $B I Q$ proved to be reliable in terms of internal consistency and test-retest stability, and there was also support for its validity as evidenced by positive correlations with observational indexes of behavioral inhibition.

Another instrument that has been specifically construed for assessing behavioral inhibition in young people is the Behavioral Inhibition Scale (BIS; Muris, Meesters, \& Spinder, 2003). The latest version of the BIS consists of 8 items covering children's shyness, fearfulness, and lack of communication and smiling when confronted with unfamiliar peers and adults. Thus, different from the BIQ (see supra), all BIS items are concerned with behavioral inhibition in the social domain. Initially, the BIS also included non-social items (e.g., "My child plays easily with the displayed toys when we are in the waiting room of an unfamiliar doctor"), but these items were removed as research indicated that they possess little validity when using the scale with older children (i.e., children aged 6-10 years; Van Brakel, Muris, \& Bögels, 2004). In other words, whereas non-social and social features both seem to be indicative for behavioral inhibition in younger children, only social signs remain predictive of this temperament-based trait when children become older (HirshfeldBecker et al., 2004; Van Brakel, Muris, \& Bögels, 2001). As such, the BIS seems appropriate to be used for measuring behavioral inhibition in children from various ages and even in adolescents.

A number of studies have examined the psychometric properties of the BIS. To begin with, the reliability (internal consistency) of the BIS appears good, with Cronbach's alphas well above 80 (e.g., Van Brakel et al., 2004; Muris et al., 2003). In a recent study by Van Brakel et al. (2004), the relation between the BIS and observable manifestations of behavioural inhibition was examined. Moderate but significant relations were found between parent- and teacher-reported behavioural inhibition of the child as measured by the BIS and an observational index of this temperamental trait, thereby providing evidence for the validity of the scale. Further, an investigation by Muris et al. (2003) showed that parent report BIS scores had acceptable correlations with children's self-report ratings of behavioral inhibition. Finally, a series of studies has demonstrated that high levels of behavioral inhibition as indexed by parent, teacher, and self-report versions of the BIS are associated with higher levels of anxiety symptoms (Muris, Merckelbach, Wessel, \& Van de Ven, 1999; Muris, Merckelbach, Schmidt, Gadet, \& Bogie, 2001; Muris et al., 2003; Van Brakel et al., 2004), a finding that also supports the validity of the scale.

The present study further examined the reliability and validity of the BIS in a sample of 7 to 12 year-old children $(N=83)$ that participated in an ongoing, longitudinal project on the role of behavioral inhibition in the development of anxiety 
disorders. More precisely, two psychometric aspects of the BIS were addressed. The first aspect pertains to the test-retest reliability of this behavior rating scale. As behavioral inhibition is considered as temperamental trait with a clear genetic basis (DiLalla, Kagan, \& Reznick, 1994; Robinson, Kagan, Reznick, \& Corley, 1992), it is not surprising that prospective research has demonstrated that children's inhibited characteristics are fairly stable over time (e.g., Asendorpf, 1994; Kagan, Reznick, \& Snidman, 1988). In this light, it seems particularly important that an instrument like the BIS, which attempts to index behavioral inhibition, should prove to possess adequate temporal stability over longer time periods. The second issue is concerned with the validity of the scale. Several authors have argued that behavioral inhibition is the perceptible manifestation of one or more underlying temperament or personality dimensions (e.g., Craske, 1997; Lonigan \& Phillips, 2001; Turner, Beidel, \& Wolff, 1996). Indeed, it can be argued that behavioral inhibition is the observable derivate of children who display high levels of negative affectivity (or neuroticism) and low levels of extraversion and regulative traits (Muris \& Dietvorst, 2006). To test this idea, the BIS was related to the Early Adolescent Temperament Questionnaire-Revised (EATQ-R; Ellis \& Rothbart, 2001), which is a scale measuring a broad range of temperamental traits, including various aspects of negative affectivity, extraversion, and regulation. It was predicted that the BIS would be positively associated with negative affectivity but negatively with extraversion and regulative traits.

\section{METHOD}

\section{Participants}

Participants were the mothers of 83 non-referred children ( 40 boys and 43 girls) who had a mean age of 8.77 years ( $S D=0.79$, range 7 to 12 years). These children participated in an ongoing longitudinal study on the role of behavioral inhibition in the pathogenesis of childhood anxiety, and had been classified as either inhibited (n $=38 ; 18$ boys and 20 girls) or non-inhibited ( $n=45 ; 22$ boys and 23 girls) at the onset of the study, some two years ago. Inhibited children had been defined by their parents as extremely shy and nervous when meeting unfamiliar persons. Noninhibited children visited the same schools as the inhibited children and were matched for gender and age. The parents of these children had indicated that their offspring did not display significant signs of behavioral inhibition. All parents gave additional informed consent for participating in the current study. More than $90 \%$ of the parents that were approached agreed to participate. 


\section{Questionnaires}

The parent version of the BIS consists of 8 items for assessing inhibited behaviours such as shyness, fearfulness, and lack of communication and smiling during encounters with an unfamiliar child (e.g., "My child is shy when he/she has to talk to an unfamiliar child") and an unfamiliar adult ("My child is shy when he/she has to talk to an unfamiliar adult"). Items are rated on 4-point Likert scales with $1=$ never, 2 = sometimes, 3 = often, and $4=$ always. After recoding positive items, scores are summed to yield a BIS child (range: 4-16), a BIS adult (range: 4-16), and a BIS total score (range: 8-32), with higher scores reflecting higher levels of behavioral inhibition.

The parent version of the EATQ-R (Ellis \& Rothbart, 2001) contains various scales that represent three main factors of children's temperament. The first factor is negative affectivity and contains the temperament traits of 'fear' 16 items; i.e., the tendency to experience unpleasant affect related to anticipation of distress; e.g., "My child worries about getting into trouble") and 'anger/frustration' ( 9 items; i.e., the tendency to experience unpleasant affect related to the interruption of ongoing tasks or goal blocking; e.g., "My child gets upset if he/she is not able to do a task really well"). The second factor is extraversion, which incorporates 'intensity pleasure' (12 items; i.e., the pleasure derived from activities involving high intensity or novelty; e.g., "My child enjoys going to places where there are big crowds and lots of excitement"), 'activity level' (6 items; i.e., participation in activities requiring high levels of physical activity; e.g., "My child would rather play a sport than watch TV"), and the reversed score of 'shyness' (7 items; i.e., behavioral inhibition to novelty and challenge, especially social; e.g., "It is a lot easier for my child to talk to familiar people than to strangers"). The third and final factor is effortful control, which includes 'activation control' ( 8 items; i.e., the ability to perform an action when there is a strong tendency to avoid it; e.g., "If my child has to do a hard task, he/she gets started right away"), 'attention control' (7 items; i.e., the ability to focus and shift attention when desired; e.g., "It is easy for my child to really concentrate on homework problems"), and 'inhibitory control' (1 1 items; i.e., the ability to plan, and to suppress inappropriate responses; e.g., "When someone tells my child to stop doing something, it is easy for him/her to stop"). The EATQ-R also contains temperament scales for measuring 'affiliation' (i.e., the desire for warmth and closeness with others; e.g., "My child enjoys exchanging hugs with people he/she likes"), 'perceptual sensitivity' (i.e., the detection and awareness of slight, lowintensity stimulation in the environment; e.g., "My child tends to notice little changes that other people do not notice"), and 'pleasure sensitivity' (i.e., the amount of 
pleasure related to activities and stimuli involving low intensity, complexity, and novelty; e.g., "My child likes to look at trees and walk amongst them"). Each item has to be answered on a 5-point Likert scale ranging from $1=$ almost never true to 5 = almost always true. Total trait and factor scores can be computed by summing ratings across relevant items (after recoding inversely formulated items).

\section{RESULTS}

\section{General findings}

Before addressing the main research issues of the present study, a number of general findings should be discussed. First of all, all questionnaires were found to be reliable in terms of internal consistency. That is, Cronbach's alphas for the BIS were all between .88 and .95, whereas these values varied between .61 and .89 for EATQ-R scales (see Table 1). Second, a second-order factor analysis performed on the EATQ-R temperament scales clearly indicated a four-factor solution that was largely in keeping with that obtained in previous research (Ellis \& Rothbart, 2001; Muris, Meesters, \& Blijlevens, submitted). The first factor consisted of activity level, intensity pleasure, affiliation, and shyness (negative loading), and hence can best be labeled as 'extraversion'. The second factor contained activation control, attention control, and inhibitory control, which all reflect the temperament factor of 'effortful control'. The third factor consisted of fear and anger/frustration and thus seemed to represent 'negative affectivity'. The fourth and final factor contained the perceptual sensitivity and pleasure sensitivity scales, and can be defined as 'low intensity sensitivity'. Together, these four factors accounted for $72.13 \%$ of the variance. Third and finally, no significant age or gender effects were found for any of the variables. 
Table 1: Mean scores (standard deviations) and internal consistency coefficients for parent versions of the BIS and the EATQ-R

\begin{tabular}{lll}
\hline & $M(S D)$ & $\alpha$ \\
\hline BIS & & \\
Total Occasion 1 & $18.61(6.28)$ & .95 \\
Child Occasion 1 & $8.45(3.27)$ & .93 \\
Adult Occasion 1 & $10.17(3.32)$ & .91 \\
Total Occasion 2 & $18.19(5.15)$ & .93 \\
Child Occasion 2 & $8.46(2.78)$ & .88 \\
Adult Occasion 2 & $9.73(2.67)$ & .89 \\
EATQ-R & & \\
Fear & & \\
Anger/frustration & $17.28(4.18)$ & .67 \\
Intensity pleasure & $27.50(5.22)$ & .71 \\
Activity level & $34.86(7.96)$ & .81 \\
Shyness & $21.05(3.87)$ & .73 \\
Activation control & $18.90(6.26)$ & .89 \\
Attention control & $26.18(5.31)$ & .80 \\
Inhibitory control & $24.54(4.63)$ & .78 \\
Affiliation & $35.13(6.23)$ & .75 \\
Perceptual sensitivity & $32.90(3.87)$ & .69 \\
Pleasure sensitivity & $19.95(3.66)$ & .61 \\
\hline Note. N & $24.67(5.06)$ & .81 \\
\hline
\end{tabular}

Note. $N=83$. BIS = Behavioral Inhibition Scale, EATQ-R = Early Adolescent Temperament Questionnaire-Revised.

\section{Stability of the BIS}

The 2-years stability of the BIS was examined in two ways. First, intra-class correlation coefficients were computed. This analysis yielded stability coefficients of .77 for the BIS Total score, .72 for BIS Child, and .71 for BIS Adult, which indicates that behavioral inhibition as measured by the BIS was fairly stable over a 2-year period. Second, a 2 (behavioral inhibition status at study onset: inhibited vs. non-inhibited) $x$ 2 (occasions) analysis of variance (ANOVA), with the last factor being a repeated measure, was performed on the BIS Total scores. This analysis yielded a main effect of behavioral inhibition status, $F(1,81)=391.92, p<.001$, Eta $^{2}=.83$, and a significant interaction effect of behavioral inhibition status and occasions, $F(1,81)=$ 28.15, $\mathrm{p}<.001$, $\mathrm{Eta}^{2}=.26$. As can be seen in Figure 1, inhibited children displayed higher BIS scores than non-inhibited children, and this appeared true on both occasions. Further, inhibited children displayed a significant increase in BIS 
scores over the two-year period [t(37) $=4.21, p<.001]$, whereas non-inhibited children showed a significant decrease $[t(44)=3.07, p<.01]$.

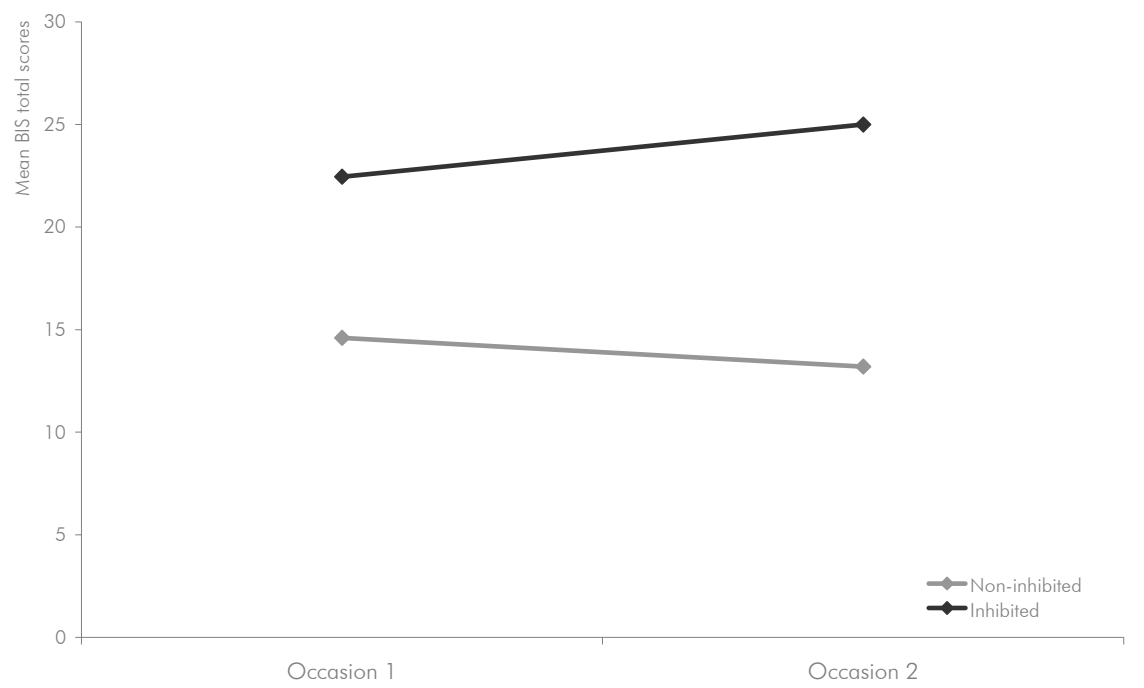

Figure 1. Mean BIS Total scores of inhibited and non-inhibited children on occasion 1 and occasion 2 (some 2 years later)

\section{Correlations between BIS and temperamental traits}

Correlations between BIS scores (on occasion 2) and EATQ-R temperament scales are displayed in Table 2. As can be seen, there were strong positive correlations between the BIS and shyness (rs between 84 and .89), which is a finding that can be taken as support for the convergent validity of the scale. Most other results were also as expected and indicated that behavioral inhibition was positively linked to fear (rs between .28 and .44) and negatively to intensity pleasure, activity level, activation control, and attention control ( $r$ between -.22 and -.46). This indicates that behavioral inhibition was positively linked to features of negative affectivity but negatively to temperamental traits related to extraversion and regulation. As a final note, small but positive correlations were found between BIS and inhibitory control (rs 
between .23 and .26), which indicates that behavioral inhibition is accompanied by a stronger tendency to plan and suppress inappropriate responses.

Table 2: Correlations between BIS scores and EATQ-R scales

BIS

\begin{tabular}{llll} 
& Total & Child & Adult \\
\hline EATQ-R & & & \\
Fear & $.39^{* *}$ & $.44^{* *}$ & $.28^{*}$ \\
Anger/frustration & -.01 & .05 & -.07 \\
Intensity pleasure & $-.45^{* * *}$ & $-.46^{* * *}$ & $-.39^{* * *}$ \\
Activity level & $-.41^{* * *}$ & $-.38^{* * *}$ & $-.40^{* * *}$ \\
Shyness & $.89^{* * *}$ & $.84^{* * *}$ & $.85^{* * *}$ \\
Activation control & $-.29^{* *}$ & $-.24^{*}$ & $-.37^{* *}$ \\
Attention control & $-.22^{*}$ & -.18 & $-.24^{*}$ \\
Inhibitory control & $.26^{*}$ & $.23^{*}$ & $.25^{*}$ \\
Affiliation & $-.28^{*}$ & -.21 & $-.33^{* *}$ \\
Perceptual sensitivity & -.02 & .02 & -.07 \\
Pleasure sensitivity & -.12 & -.12 & -.10 \\
\hline
\end{tabular}

Note. $N=83$. BIS = Behavioral Inhibition Scale, EATQ- $R=$ Early Adolescent Temperament

Questionnaire-Revised. ${ }^{*} p<.05,{ }^{* *} p<.01,{ }^{* * *} p<.001$.

\section{DISCUSSION}

The BIS is a brief rating scale for measuring behavioral inhibition in children. The current study further examined the reliability and validity of the BIS in a sample consisting of inhibited children and non-inhibited children. Results indicated that BIS scores were fairly stable over a 2-year period, with a test-retest correlation of .77. Further, BIS scores of inhibited children significantly increased over the 2-year period, whereas those of non-inhibited children significantly decreased. Finally, BIS scores were in a theoretically meaningful way associated with Rothbart's temperament scales, which provides support for the validity of the scale.

In their review article on behavioral inhibition, Hirshfeld-Becker et al. (2004) list a series of studies that examined the stability of behavioral inhibition (Asendorpf, 1990, 1994; Broberg, Lamb, \& Hwang, 1990; Fordham \& StevensonHinde, 1999; Kagan et al., 1988; Kerr, Lambert, Stattin, \& Klackenberg-Larsson, 1994; Scarpa, Raine, Venables, \& Mednick, 1995; Stevenson-Hinde \& Shouldice, 1995) and come to the conclusion that test-retest correlations for this construct range 
between .11 and .73 , indicating low to fair stability over substantial periods of time. The test-retest correlations as found in the present study compare rather favorable with these figures. However, it should be kept in mind that the test-retest period was relatively short (i.e., 2 years). Further, the current study relied on selected samples of inhibited and non-inhibited children, for which typically somewhat higher stabilities of behavioral inhibition are found (Hirshfeld-Becker et al., 2004).

The finding that the groups diverged over time (that is, BIS scores of inhibited children increased whereas those of non-inhibited children decreased over the 2-year period) strengthens the notion that non-inhibited children learn how to cope with difficult (social) situations, whereas inhibited children tend to avoid these situations and as a result do not acquire the skills to deal with such events and become even more inhibited over time (Hirshfeld-Becker et al., 2004).

Several authors have argued that behavioral inhibition is the perceptible manifestation of one or more underlying temperamental traits (Craske, 1997; Lonigan \& Phillips, 2001; Turner et al., 1996). A previous study by Muris and Dietvorst (2006) found some evidence for the idea that behavioral inhibition is based on high levels of negative affectivity (i.e., fear) and low levels of regulation (i.e., activation control, attention control). The present data are in keeping with this notion, but also indicate that low levels of extraversion (i.e., activity level, high intensity pleasure) are also involved. Altogether, these results provide further insight in the temperamental structure behind the observable individual difference factor of behavioral inhibition.

On first sight, it might be surprising that inhibitory control was positively linked to behavioral inhibition. As this temperamental characteristic belongs to the regulative traits, a negative correlation could have been anticipated. Note, however, that inhibitory control, which is defined as the capacity to plan, and to suppress inappropriate responses, fits rather well with the phenomenon of behavioral inhibition. Inhibited children typically display excessive self-control, which means that they cautiously plan and try to avoid improper behavior (Wenar \& Kerig, 2000).

In conclusion, then, the BIS seems to be a brief, reliable, and valid scale for assessing behavioral inhibition in children. As such, the scale provides an alternative for the time-consuming behavioral observation procedures that are generally employed to assess this temperament-based construct. Researchers and clinicians who want to measure children's level by means of a rating scale have the choice between the BIS and Bishop et al.'s (2003) BIQ. The main difference between these questionnaires is that the $B I Q$ not only includes items referring to social stimuli, but also contains items that pertain to behavioral inhibition to non-social stimuli. This 
makes the BIQ more appropriate to be used with younger (i.e., preschool) children. For older children, the BIS seems an attractive alternative: the scale is briefer and there is also a self-report version available (see Muris et al., 1999, 2001, 2003) on which children themselves can rate their own level of behavioral inhibition. 




\section{CHAPTER 4}

\section{Eye blink startle responses in}

behaviorally inhibited and uninhibited children

This chapter appeared as: Van Brakel, A. M. L., Muris, P. \& Derks, W. (2006). Eye blink startle responses in behaviorally inhibited and uninhibited children. International Journal of Behavioral Development, 30, 460-465. 


\section{ABSTRACT}

The present study examined the startle reflex as a physiological marker of behavioral inhibition. Participants were 7- to 12-year-old children who had been previously identified as inhibited or uninhibited as part of an ongoing longitudinal study on the role of behavioral inhibition in the development of anxiety disorders. Analysis of their scores on the Behavioral Inhibition Scale revealed that the children were stable in their behavioral inhibition categorization as compared to the beginning of the longitudinal study. An experiment was carried out to study startle modulation effects in response to novel and familiar pictures of threatening and non-threatening facial expressions in inhibited and uninhibited children. The main results can be summarized as follows. To begin with, no modulation effect was found. That is, children did not show the expected (adult-like) startle facilitation while viewing unpleasant pictures. Second, a habituation effect was found: that is, during the testing phase children responded more intensely to the first block of slides than to the second block of slides (irrespective of slide content). Third, unexpectedly behaviorally inhibited children displayed smaller eye blink magnitudes in response to novel slides than uninhibited children. Fourth and finally, no meaningful differences were found in the patterns of startle responses of both genders. 


\section{EYE BLINK STARTLE RESPONSES IN BEHAVIORALLY INHIBITED AND UNINHIBITED CHILDREN.}

Epidemiological studies have reported estimates for the presence of any anxiety disorder ranging between 5 and 20\% (for a review see Costello \& Angold, 1995). As such, anxiety disorders can be considered as one of the most common psychiatric diagnoses among children and adolescents (Craske, 1997). In view of these relatively high prevalence rates, it seems important to study the factors that contribute to the development of anxiety disorders in youths (March, 1995). One dispositional variable that is thought to be involved in pathogenesis of childhood anxiety disorders is the temperamental construct of behavioral inhibition. Children who are characterized by a behaviorally inhibited temperament tend to display fear and withdrawal in situations that are novel or unfamiliar (Kagan, Reznick, \& Snidman, 1988). Research has indeed provided evidence showing that these children are at increased risk for developing anxiety disorders. For example, Biederman and colleagues (1990) found that inhibited children, compared to uninhibited children, more frequently displayed the clinical symptoms of multiple anxiety disorders. Furthermore, in a longitudinal follow-up study (Biederman, Rosenbaum, BolducMurphy et al., 1993), this group of researchers noted that pre-school children initially identified as behaviorally inhibited were more likely to have developed anxiety disorders at 3-years follow-up compared to control children (i.e., children who at study onset were not classified as behaviorally inhibited). Additional support for a link between behavioral inhibition and childhood anxiety comes from a series of studies conducted by Muris and colleagues $(1999,2001,2003)$. In these studies, youths and their parents completed a questionnaire measuring children's behaviorally inhibited temperament. Results showed that children who were identified as high on behavioral inhibition displayed higher levels of anxiety as compared to children who were classified as low on behavioral inhibition. All the abovementioned studies suggest that behavioral inhibition is associated with the development of high levels of anxiety symptoms and anxiety disorders.

In the literature, it has been suggested that behavioral inhibition has a clear-cut biological basis (Kagan et al., 1988). More precisely, a behavioral profile like behavioral inhibition may be defined by inherited physiological processes, which predispose children to display these particular emotions and behaviors (Kagan, Snidman, \& Arcus, 1993). A number of studies support the hypothesis that behaviorally inhibited children are born with a lower threshold for subcortical arousal to unexpected changes in the environment or novel events that cannot be easily 
assimilated (Garcia Coll, Kagan, \& Reznick, 1984; Kagan et al., 1988; Kagan \& Snidman, 1991; Schmidt \& Fox, 1998; Schmidt, Fox, Rubin et al., 1997; Snidman, 1989; for similar results in animal research see Adamec \& Stark-Adamec, 1989).

The brain areas that are particularly relevant in this context are the limbic system, the hypothalamus, and the amygdala. The limbic system is associated with a broad range of emotions and processes information from cortical, subcortical, endocrine, and autonomic systems (Partridge, 2000). Among other things, the hypothalamus comes into play in the case of stress. The amygdala is involved in the processing of fear-related stimuli and as such may be especially relevant for understanding the brain mechanisms underlying behavioral inhibition. One way to demonstrate that behavioral inhibition is linked to heightened sensitivity of the amygdala is to use the eye blink startle reflex. This notion is based on a number of empirical findings. To begin with, it has been demonstrated that lesions in the central nucleus of the amygdala block the expression of fear-potentiated startle reflexes (Hitchcock \& Davis, 1986, 1987). In addition, various studies have shown that children who are at risk for anxiety disorders (and hence frequently show the signs of behavioral inhibition) have elevated startle reflexes (Grillon, Dierker \& Merikangas, 1997; Merikangas, Avenevoli, Dierker \& Grillon, 1999), which suggests that the startle probe paradigm may be a useful method for studying the biological origins of behavioral inhibition (Schmidt \& Fox, 1998). This paradigm seems particularly relevant for two reasons. First, the amplitude of the startle response is thought to reflect the underlying emotional state of an organism (Lang, 1995). Second, the startle response may tap individual differences in the excitability of the amygdala (Schmidt \& Fox, 1998). As such the startle response might be linked to individual differences in human temperament and personality traits. There is indeed some support for these ideas. Snidman en Kagan (1994) reported that inhibited children, as compared with uninhibited children, generally displayed augmented startle responses in response to auditory probes, regardless of the affective content of the slides they were presented with. Thus, if the origins of behavioral inhibition are in part due to a relatively low threshold for arousal in the amygdala, then children who display the characteristics of this temperamental disposition should display an increase in startle amplitude in response to fear-eliciting situations (Schmidt \& Fox, 1998).

The primary feature of behavioral inhibition is concerned with the response to novelty, which hence is independent of threat. This is quite different from social anxiety, which predominantly has to do with the threat of embarrassment in social situations and not necessarily with the avoidance of novel social cues. Schwartz, 
Wright, Shin et al. (2003) carried out an experiment during which they assessed fMRI responses to pictures of novel and familiar faces with neutral expressions. Results showed that the amygdala responded more intensely to novel, neutral faces as compared to familiar, neutral faces. Moreover, they found that adults who had been categorized in the second year of their life as behaviorally inhibited showed greater FMRI responses in the amygdala when confronted with novel faces than adults who had been previously classified as uninhibited (Schwartz, Wright, Shin, Kagan, \& Rauch, 2003).

Sensitive physiological markers such as the startle reflex might distinguish children with similar phenotypes into distinct groups of children who are shy or fearful as a result of a temperamental predisposition (i.e., behavioral inhibition) or as a result of environmental circumstances (Kagan, Snidman, McManis, Woodward, \& Hardway, 2002), and thus may prove useful in the identification of children who are actually at risk for developing anxious psychopathology (Woodward, McManis, Kagan, Deldin, Snidman, Lewis, \& Kahn, 2001). With this in mind, the present study further examined the startle reflex as a physiological marker of behavioral inhibition. For this purpose a number of the aforementioned paradigms were combined. That is, we not only examined startle modulation effects to threatening facial expressions in inhibited and uninhibited children, but we also added a condition in which the children were confronted with pictures of familiar and novel faces. It was hypothesized that inhibited children compared with uninhibited children would show a greater modulation effect in response to threatening faces (i.e., a facilitation of startle responses). Moreover, it was expected that the inhibited children would show augmented startle blink reflexes while viewing pictures of novel faces rather than when looking at pictures of familiar faces. Participants were 7- to 12-year-old children who had been previously identified as inhibited or uninhibited as part of an ongoing longitudinal study on the role of behavioral inhibition in the development of anxiety disorders.

\section{METHOD}

\section{Participants}

Participants were 83 children that were categorized as inhibited ( $n=38 ; 18$ boys and 20 girls) or non-inhibited ( $n=45 ; 22$ boys and 23 girls) some two years ago. Children participated in an ongoing longitudinal study on the role of behavioral inhibition in the development of anxiety disorders (Van Brakel, Muris \& Bögels, 2001). Inhibited children had been defined by their parents as extremely shy and 
nervous when meeting unfamiliar persons. Non-inhibited control children visited the same schools as the inhibited children and were matched for gender and age. The parents of these children had indicated that their offspring did not display significant signs of behavioral inhibition. Parents of all children gave additional informed consent for participating in the current study. The mean age of the children was 8.8 years $(S D=.79): 1.2 \%$ was 7 years old, $37.3 \%$ was 8 years old, $47.0 \%$ was 9 years old, $13.3 \%$ was 10 years old, and the remaining $1.2 \%$ was 12 years old.

\section{Apparatus and Procedure}

The startle reflex was recorded with a Picker Schwarzer ED14/12. The acoustic stimulus was a 50 -ms duration $95 \mathrm{~dB}$ burst of white noise presented binaurally through headphones. The sound level was calibrated using a GenRad GR1567 Sound Level Calibrator. The orbicularis oculi electromyographic (EMG) activity was recorded with two disk electrodes $(\mathrm{Ag} / \mathrm{AgCl})$ placed underneath the left eye. The reference electrode was placed on the forehead. Figure 1 shows an example of a startle response as recorded electro-physiologically.

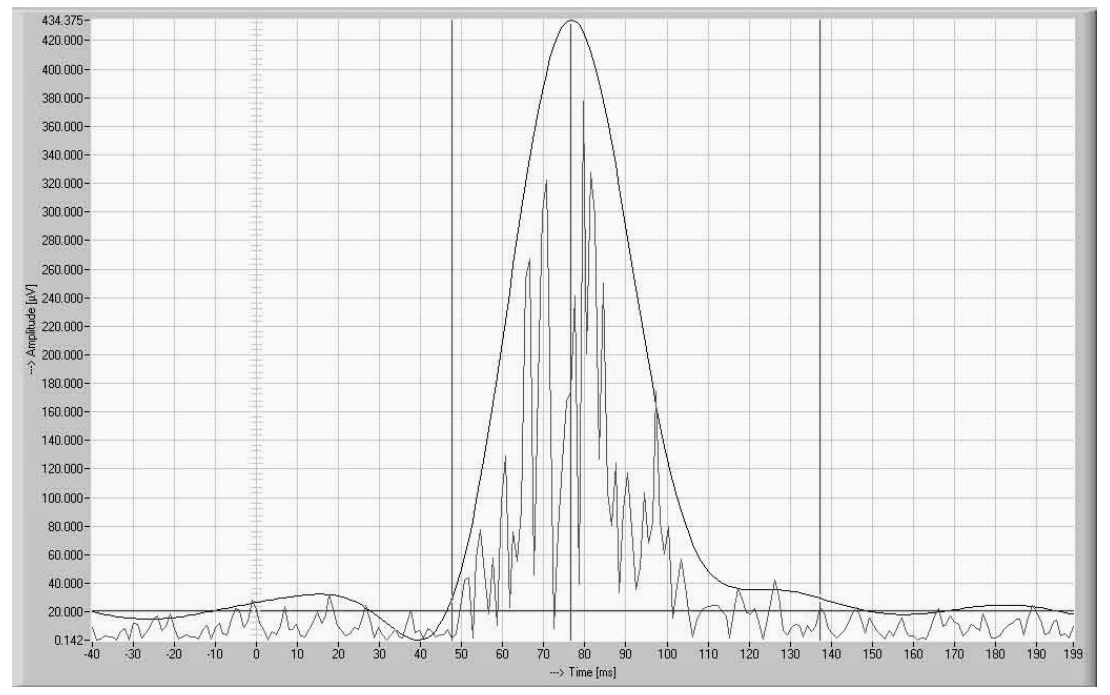

Figure 1. An example of a startle response taken from this study, as recorded electrophysiologically 
Children who participated in a longitudinal project examining behavioral inhibition as a risk factor for the development of anxiety disorders in children were sent a letter providing them with information about this startle reflex study. Next, parents of these children were contacted by phone in order to give them additional information about the study. Eighty-three children and their parents decided to participate, yielding a response rate of more than $90 \%$.

Children visited the lab one by one together with one of their parents (usually the mother). During testing, parents stayed outside the experimental room and in the meantime filled out parent versions of the Behavioral Inhibition Scale (BIS; Muris et al. 1999). In the experimental room, the child was seated in a chair behind a table on which a computer screen was placed. Next, the child received experimental instructions. He/she was told that he/she was going to watch a series of slides depicting human faces with different expressions on the computer screen and that he/she had to look very carefully. Instructions concerning the white noise ran as follows: "Now and then you will hear a loud noise through the headphones, but you don't have to pay any attention to this".

The experiment consisted of three phases: (1) a baseline startle phase, (2) a familiarization phase, and (3) a test phase with 2 blocks of either novel or familiar faces. During the first phase, children were asked to watch the computer screen. There was nothing to be seen on the screen, but during 24 seconds the startle response was elicited four times by a $50 \mathrm{~ms}$ burst of white noise of $95 \mathrm{~dB}$. This baseline startle procedure was performed to reduce initial startle reactivity. During the 180-seconds familiarization phase, children were presented with 72 slides. The series of slides consisted of 4 presentations of 6 different faces in 3 different expressions that were shown in random order. The series was preceded by a brief 6 seconds waiting period during which children saw a small fixation cross on the spot where the slides were going to appear. Each picture was shown for 2 seconds and the interstimulus interval was $500 \mathrm{~ms}$ (see also Schwartz et al., 2003). After a second, brief 6-seconds waiting period, the test phase took place. There were two 216seconds blocks of slides, one showing 18 different novel faces, 6 with a neutral expression, 6 with an unpleasant (angry) expression, and 6 with a pleasant (happy) expression. The novel pictures were only shown once and were never repeated. The other block showed 18 familiar faces (i.e., faces that had been previously presented during the familiarization phase), again 6 for each emotional expression. During this phase, pictures were shown for 6 seconds and the interstimulus interval was 6 seconds (see also Balaban \& Taussig, 1995). For one half of the children (balanced for gender and inhibition category), the test phase started with the novel faces block, 
whereas for the other half of the children the test phase started with the familiar faces block. During the test phase, the startle blink response was elicited 18 times, 9 times during each block. Presentations were random but balanced for the content of the pictures (i.e. male/female face and emotional expression). The acoustic startle stimuli were presented randomly between 3 and 5 seconds after slide onset. The slides used in this study were taken from 3 sources: (1) pictures available from Ekman and Friesen (1975), (2) pictures taken from the International Affective Picture System (IAPS; Lang, Öhman, \& Vaitl, 1988) and, (3) 6 pictures were made by ourselves and validated by means of IAPS ratings. All pictures were visually comparable. That is, pictures were digitally adjusted in such a way that they all had the same size on the screen. Further, all faces were of comparable size and had a similar contrast level relative to the background.

\section{TEMPERAMENT MEASUREMENT}

Behavioral Inhibiton Scale (BIS). Inspired by the work of Reznick, Hegeman, Kaufman, and Woods (1992) and Gest (1997), Muris et al. (1999) developed the BIS, a brief self-report questionnaire for assessing behavioral inhibition in children. The BIS consists of 4 items: shyness ("I am shy when I have to talk to an unfamiliar person"), communication ("I talk easily to an unfamiliar person"), fearfulness ("I feel nervous when I have to talk to an unfamiliar person"), and smiling ("I feel good and I am able to laugh, when I talk to an unfamiliar person"). The parent version of this questionnaire contains items referring to "an unfamiliar child" and "an unfamiliar adult" (e.g., "My child talks easily to an unfamiliar child", "My child talks easily to an unfamiliar adult") (see also Van Brakel, Muris \& Bögels, 2004), and thus contains 8 items. Each item is scored on a 4-point Likert scale: 1 means never, 2 sometimes, 3 often, and 4 always. After recoding the positive items, scores are summed to yield a total BIS score, ranging from 8 (not apprehensive, not shy and very sociable when meeting unfamiliar persons) to 32 (very apprehensive and shy and not capable of initiating social interaction with unfamiliar persons). Previous research has yielded support for the reliability and validity of the BIS. To begin with, in a recent study by Van Brakel et al. (2004), the relation between the BIS and observable manifestations of behavioral inhibition was examined. Moderate but significant relations were found between parent- and teacher-reported behavioral inhibition of the child as measured by the BIS and an observational index of this temperamental trait, thus providing evidence for the validity of the scale. Further, an investigation by Muris et al. (2003) showed that self-report BIS scores had acceptable correlations with parent ratings on 
the BIS. Finally, the reliability of the BIS (internal consistency) appears good, with Cronbach's alphas well above .80 (e.g., Muris et al., 2003; Van Brakel et al., 2004). In the current study, the BIS also proved to be reliable, with a Cronbach's alpha of .93.

\section{DATA REDUCTION AND ANALYSIS}

A number of standard procedures were followed to determine the magnitude of the startle response. The baseline level was defined as the overall mean of the data occurring between $-40 \mathrm{~ms}$ and $+10 \mathrm{~ms}$ around $t=0(t=0$ is the moment the stimulus was presented). The top amplitude was defined by the maximum of the data within a framework of 15 to $150 \mathrm{~ms}$ from $t=0$. The startle response had to occur between 15 to $75 \mathrm{~ms}$ from $t=0$. The startle magnitude was computed by subtracting the baseline level from the top amplitude.

All startle responses were carefully inspected to control whether they met the above described criteria. In other words, responses with an onset outside the boundaries of 15 to $75 \mathrm{~ms}$ from $t=0$ were discarded. Further, the top of the response had to occur within $100 \mathrm{~ms}$ from onset, and the onset amplitude had to be at most $4 \%$ above the baseline level.

To examine the pattern of startle changes among conditions, Z-scores were used to adjust for within-subject variations in startle magnitude. Following Grillon and Ameli (1998), the raw scores for each subject were deviated from the individual's mean score and divided by his or her standard deviation using the 18 average startle scores. The Z-scores were then transformed into T-scores by multiplying each score by 10 and then adding 50. These data were entered into a repeated-measures analysis of variance (ANOVA) with Group (inhibited vs. not inhibited) and Condition (novel-familiar vs. familiar-novel) as between-subject factors and Valence (neutral vs. happy vs. angry) and Slide (novel vs. familiar) as withinsubject factors. Data of 56 children were used in the analysis. That is, for 25 children ( 12 from the uninhibited group and 13 from the inhibited group) data were not used because they had excessive startle responses off scale (that is, these children displayed physiological responses that did not meet all the criteria of a startle) and 2 of the children were too anxious and refused to complete the procedure. 


\section{RESULTS}

Intra-class correlation coefficients were computed in order to examine the 2-years stability of the BIS. This analysis yielded a stability coefficient of .77 for the BIS Total score, which indicates that behavioral inhibition as measured by the BIS was fairly stable over a 2-year period. Furthermore, it was found that inhibited children displayed a significant increase in BIS scores over the 2-year period, whereas noninhibited children showed a significant decrease (Van Brakel \& Muris, 2006). Moreover, $97.6 \%$ of the children remained in the same category (i.e., inhibited or uninhibited) from time point 1 to time point 2 (kappa $=$. 95).

First of all, it was expected that the results would show a main effect of Valence, indicating a modulation effect. Secondly, it was hypothesized that inhibited children compared with uninhibited children would show a greater modulation effect, that is, an interaction effect of Group x Valence. Moreover, it was expected that the inhibited children would show an augmented startle blink reflex while watching pictures of novel faces rather than when looking at pictures of familiar faces, that is, an interaction effect of Group x Valence $\times$ Slide.

A repeated-measures analysis of variance (ANOVA) was performed and yielded none of the expected main and interaction effects. That is, the results did not show a main effect of Valence, indicating that there was no modulation effect $[F(1,52)=1.74, p=.20]$. Further, neither the Group $\times$ Valence interaction $[F(1,52)$ $=3.36, p=0.07]$ nor the Group $\times$ Valence $\times$ Slide interaction $[F(1,52)=1.12, p=$ .30] was found.

Furthermore, the results indicated three significant interaction effects. First, a Slide $\times$ Condition interaction was found $[F(1,52)=75.11, p<.001]$. Regardless of the content of the slides children reacted with larger eye blink reflexes to the first series of slides during the test phase. That is, children who were assigned to the condition familiar-novel reacted with a greater amplitude to the familiar slides $(t=$ $7.44 ; p<.001)$, whereas children who were assigned to the condition novel-familiar reacted with a greater amplitude to the novel slides $(t=4.68 ; p<.001)$. This seems to reflect a clear-cut habituation effect. Second, a significant Slide $x$ Group interaction was found $[F(1,52)=4.02, p=.05]$. Surprisingly, behaviorally inhibited children generally showed smaller startle reflex amplitudes in response to novel slides, particularly happy faces, than behaviorally uninhibited children $(t=2.05 ; p<$ .05). Third, a significant Slide $\times$ Valence interaction emerged $[F(1,52)=4.12, p<$ .05], which indicates that the eye blink magnitudes for the three valences were different for the novel slides as compared to the familiar slides. More specifically, in 
response to the slides with the happy faces, children showed larger eye blink magnitudes in the novel condition, whereas when exposed to angry and neutral faces eye blinks were larger in the familiar condition $[F(1,52)=4.90, p<.05$ and $F(1,52)$ $=4.12, p<.05$, respectively]. No difference in eye blink magnitudes was observed when comparing children's eye blink magnitudes for the neutral and angry faces in both conditions $[F(1,52)=.01, p=.91]$.

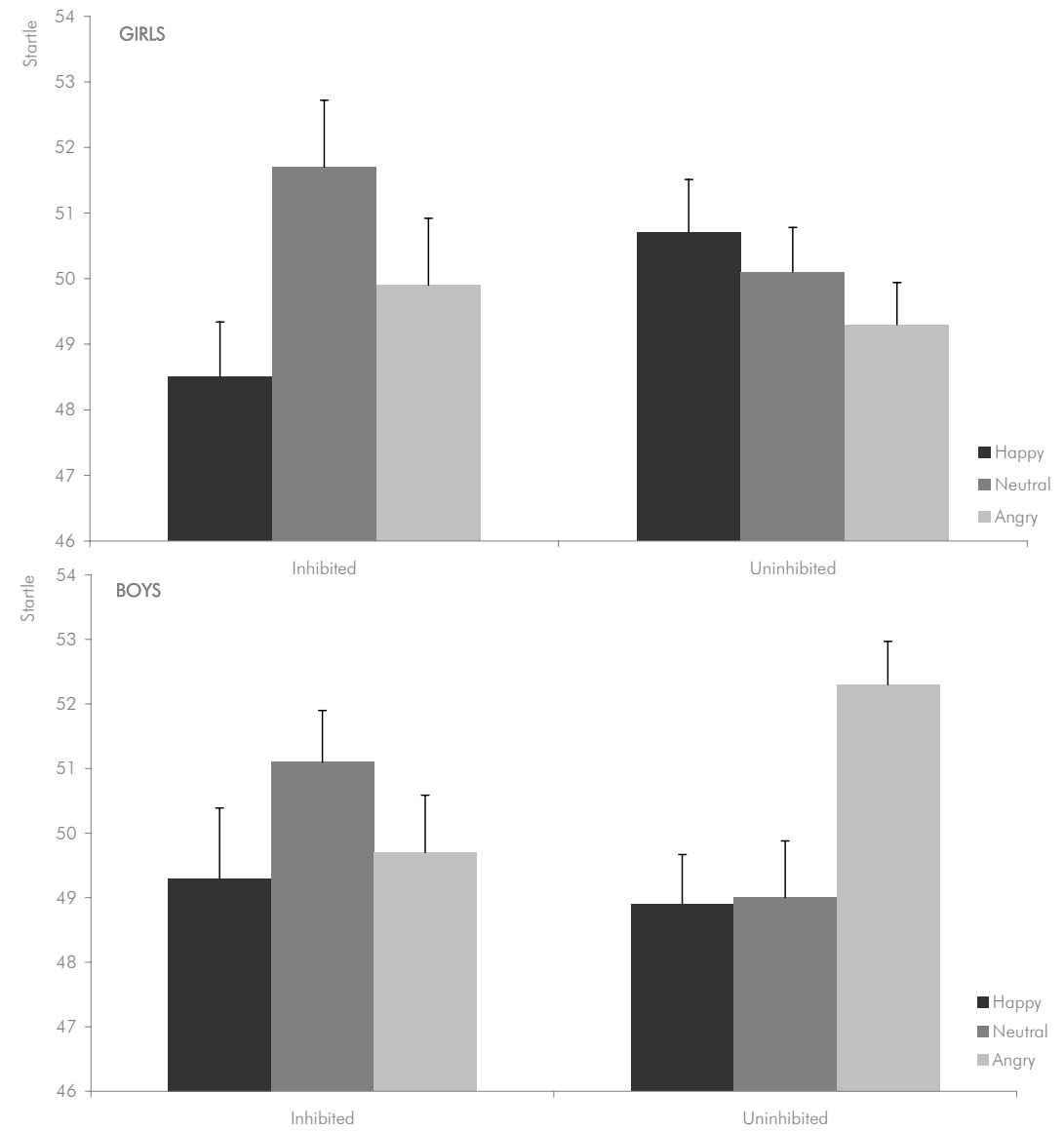

Figure 2: Mean eye blink startle magnitudes (and standard errors) to slides with happy, neutral, and angry facial expressions in inhibited $(N=25)$ and uninhibited $(N=37)$ children, computed for boys and girls separately 
Finally, separate analyses were carried out for both genders, following McManis, Bradley, Cuthbert, and Lang (1995) who found the expected (adult-like) startle modulation effect in girls, whereas boys actually showed an inhibition of the startle reflex. In girls, the analyses yielded the predicted Valence and Valence $x$ Group effects, respectively $F(1,27)=4.32, p<.05$ and $F(1,27)=5.10, p<.05$. However, as can be seen in Figure 2, these effects were not in keeping with the hypothesized pattern.

\section{DISCUSSION}

The current study investigated the startle reflex as a physiological marker of behavioral inhibition in children. The main results can be catalogued as follows. To begin with, no modulation effect was found. That is, children did not show the expected (adult-like) startle facilitation while viewing unpleasant pictures. Second, a habituation effect was found, which means that during the testing phase children responded more intensely to the first block of slides than to the second block of slides (irrespective of slide content). Third, unexpectedly, behaviorally inhibited children displayed smaller eye blink magnitudes in response to novel slides than uninhibited children. Fourth and finally, when studying the startle responses of both genders separately, the data did not reveal any of the anticipated effects in girls or boys.

In part, the present findings are in line with those of previous studies. In a study examining the psychophysiological basis of self-presentation anxiety, Schmidt, Fox, Schulkin, and Gold (1999) failed to document larger eye blink magnitudes in temperamentally shy children who were confronted with a socially evaluative situation. McManis et al. (1995) were also unable to demonstrate startle facilitation to pictures with negative affective valence in 7- to 10-year-old children. However, when responses were segregated by gender, girls did show the expected facilitation of startle reflexes while viewing unpleasant pictures, whereas boys actually showed an inhibition of startle responses. In a similar vein, Cook, Hawk, Hawk, and Hummer (1995) found a significant affective valence by fear interaction, but this interaction was precisely opposite to that found in adults. That is, the startle responses of children who scored higher on a fear survey schedule were smaller during unpleasant imagery than during pleasant imagery, and were generally smaller in the "high fear" than in the "low fear" children. In an attempt to explain these unexpected results, Ornitz (1999) states that it may well be that fear-prone children devote greater attention to unpleasant pictures and engage more in unpleasant imagery, which requires so much attentional resources that it interferes with the processing of 
the startling stimuli, resulting in smaller startle responses. In addition, it is possible that our instructions about the startle probes ("Now and then you will hear a loud noise through the headphones, but you don't have to pay any attention to this") might have strengthened this effect.

Some alternative explanations can be put forward for not finding the hypothesized startle effects. First, the affective modification of the startle responses might only occur when the arousal component of emotion is sufficiently high (Lang, 1995). It may be the case that in our study, the emotional content of the slides was not impressive enough in order to elicit high (positive or negative) arousal and that as a result no differential startle responses occurred. Second, it is possible that inhibited children displayed a greater tendency to avoid the slides by just not looking carefully at them. As we did not record the sessions in the lab on video, it is not possible to check this possibility.

A drawback of this study pertains to the fact that data of 27 children $(32.5 \%)$ could not be included in the analysis. A comparison of this percentage with those reported in previous studies using the startle paradigm, shows that it seems quite normal to have this amount of drop-outs due to technical issues (i.e., Bradley, Cuthbert, \& Lang (1990), 14.3\%; Schmidt \& Fox (1998), 68.5\%; Schmidt et al. (1997), 50.8\%).

As Cook and colleagues (1991) already agreed upon: it appears that multiple processes (including selective attention and a process related to affective valence) modulate the magnitude of startle reflexes. Moreover, in children we have to take their developmental phase (cognitive as well as neurophysiological processes) into account when studying such complicated psycho-physiological phenomena. Thus, although the age range of the children in the present study was small, there may have been important between-subject differences because of variation in their brain maturation and neurophysiological development.

The observation that till now mixed results have dominated the studies of behaviorally inhibited children's startle responses (i.e., compare our results to Snidman \& Kagan, 1994) let us to conclude that the startle reflex paradigm might not be the right procedure to measure the excitability of the amygdala in (inhibited) children. fMRI may be a more useful and direct tool for identifying the biological underpinnings of children at risk for anxiety disorders (Vasa \& Pine, 2004). 



\section{CHAPTER 5}

A multifactorial model for the etiology of anxiety in non-clinical adolescents: Main and interactive effects of behavioral inhibition, attachment, and parental rearing

This chapter appeared as: Van Brakel, A. M. L., Muris, P., Bögels, S. M. \& Thomassen, C. (2006). A multifactorial model for the etiology of anxiety in non-clinical adolescents: Main and interactive effects of behavioural inhibition, attachment, and parental rearing. Journal of Child and Family Studies, 15, 568-578. 


\begin{abstract}
There has been limited research examining the additive and interactive effects of multiple factors on the development of anxiety symptoms and anxiety disorders in youths. The present study was an attempt to examine the reciprocal connections among temperament, attachment, and rearing style, and their unique and interactive relations to anxiety symptoms. Six hundred forty-four non-clinical children aged 11 15 years (mean age $=12.7$ years) completed questionnaires measuring behavioral inhibition, attachment, parental rearing behavior, and anxiety symptoms. Results indicated that there were small to moderate positive correlations among various risk factors. Furthermore, modest but significant positive correlations were found between behavioral inhibition, attachment quality, and anxious and controlling rearing behaviors, on the one hand, and anxiety scores, on the other hand. That is, higher levels of behavioral inhibition, insecure attachment, and parental control and anxious rearing were associated with higher levels of anxiety symptoms. Finally, behavioral inhibition, attachment quality, parental control and anxious rearing each accounted for a small but unique proportion of the variance of anxiety disorders symptomatology. Little support was found for interactive effects of these vulnerability factors on childhood anxiety.
\end{abstract}



REARING

Anxiety disorders are among the most prevalent psychiatric problems in children and adolescents (Bernstein, Borchardt, \& Perwien, 1996; Craske, 1997). A review of 16 epidemiological studies (1988-1995) shows estimates for the presence of any anxiety disorder ranging between 5.7 and $17.7 \%$, with half of them exceeding the $10 \%$ rate (Costello \& Angold, 1995). The interest for research on anxiety disorders in children has grown enormously during the past two decades (Muris, 2006). A number of these studies have been devoted to the etiology and are thus concerned with the question of which factors contribute to the development and maintenance of anxiety disorders in children and adolescents.

One dispositional variable that is thought to be involved in the pathogenesis of anxiety disorders is behavioral inhibition. Children who are characterized by a behaviorally inhibited temperament tend to display fear and withdrawal in situations that are novel or unfamiliar (Kagan, Reznick, \& Snidman, 1988). Research has shown that these children seem to be at risk for developing anxiety disorders. Findings of Biederman et al. (1990) indicate that inhibited children, compared to uninhibited children, more frequently displayed the clinical symptoms of multiple anxiety disorders. Moreover, in a longitudinal study, Biederman, Rosenbaum, Bolduc-Murphy, and Faraone (1993) found that pre-school children initially identified as behaviorally inhibited were more likely to have developed anxiety disorders at 3-years follow-up compared to control children (i.e., children who at study onset were not classified as behaviorally inhibited). Further support for a link between behavioral inhibition and anxiety comes from a series of studies conducted by Muris and colleagues (1999, 2001b, 2003a). In these studies, adolescents and their parents completed a questionnaire measuring children's behaviorally inhibited temperament. Results showed that children who were identified as high on behavioral inhibition displayed higher levels of anxiety compared to children who were classified as low on behavioral inhibition. All the abovementioned studies suggest that behavioral inhibition is associated with the development of a broad range of anxiety symptoms and anxiety disorders.

Besides temperament, attachment theory provides an explanation for the development of anxiety disorders in children. For example, in their longitudinal study, 
Warren and colleagues (1997) found a specific link between insecure (in particular, ambivalent) attachment assessed in infancy and anxiety disorders assessed some 16 years later. A study by Manassis, Bradley, Goldberg, Hood, and Swinson (1994) examining attachment in mothers with anxiety disorders and their children, showed that all children with diagnosed anxiety disorders were insecurely attached. Results of another study by this research group demonstrated that insecurely attached children had higher levels of internalizing problems than their securely attached counterparts (Mannasis, Bradley, Goldberg, Hood, \& Swinson, 1995). In three subsequent studies, Muris and colleagues (Muris, Mayer, \& Meesters, 2000a; Muris, Meesters, Merckelbach, \& Hülsenbeck, 2000b; Muris, Meesters, Van Melick, \& Zwambag, 2001a) found that adolescents who defined themselves as insecurely attached displayed higher levels of anxiety and worry than youths who defined themselves as securely attached.

Another factor that seems to play a role in the origins of anxiety is parental rearing behavior. Two parenting dimensions that have been repeatedly associated with the development of anxiety in children and adolescents are control and anxious rearing (e.g., Wood, McLeod, Sigman, Hwang, \& Chu, 2003). Control can be best described as parental behaviors aimed at guiding the child during daily activities. These parental behaviors often have the effect of directing the child and reducing the development of autonomy (Rapee, 1997). Anxious rearing pertains to the explicit encouragement of anxious cognitions and avoidance behaviors in children (e.g., Barret, Rapee, Dadds, \& Ryan, 1996; Grüner, Muris, \& Merckelbach, 1999). Several studies have found confirming evidence for the proposed relationship between controlling rearing behaviors and anxiety disorder symptoms, some of them relying on direct observation of parent-child interactions (Hudson \& Rapee, 2001; Whaley, Pinto, \& Sigman, 1999) and others making use of questionnaires that intend to measure children's perceptions of parental rearing behaviours (Grüner et al., 1999; Muris et al., 2000b; Muris, Meesters, \& Van Brakel, 2003b).

Vasey and Dadds (2001) describe an integrative framework for conceptualizing the various pathways associated with the development of childhood anxiety disorders. The core issue in this model is the dynamic interplay of various potential predisposing factors in the pathogenesis of anxiety. Studies examining additive and interactive effects of these factors on childhood anxiety are just beginning to emerge. For example, Calkins and Fox (1992) obtained evidence for a reciprocal relation between attachment style and behavioral inhibition. That is, in their sample of young infants, insecure attachment appeared to promote behavioral inhibition, whereas behavioral inhibition seemed to hinder the formation of a secure 
attachment relationship, thereby further enhancing children's vulnerability to anxiety. Mannasis et al. (1995) found that within a group of inhibited children, the children with an insecure attachment showed higher levels of anxiety symptoms in comparison to the children with a secure attachment. Muris et al. (2000b) found that adverse parental rearing and insecure attachment in primary school children were both positively linked to symptoms of worry. Moreover, the results of this study showed that there were significant associations between parental rearing and attachment style and that both factors accounted for independent variance in worry scores. The results of a similar study in young, non-clinical adolescents (Muris \& Meesters, 2002) indicate that attachment and behavioral inhibition both play a unique role in the manifestation of childhood anxiety.

Altogether, so far, there has been limited research examining the additive and interactive effects of multiple risk factors on the development of anxiety symptoms and anxiety disorders in youths. The present study was an attempt to examine the reciprocal relations among inhibited temperament, insecure attachment, and anxious and controlling rearing behaviors, and their contribution to anxiety. Six hundred forty-four non-clinical children completed questionnaires measuring behavioral inhibition, insecure attachment, parental rearing behavior, and DSMdefined anxiety symptoms. It was hypothesized that behavioral inhibition, insecure attachment, and a controlling or anxious rearing style independently predict adolescents' anxiety symptoms. Moreover, we examined the interactive effects of these risk factors on children's anxiety symptoms.

\section{METHOD}

\section{Participants and procedure}

Participants were 644 children (337 boys and 307 girls) recruited from regular secondary schools in The Netherlands. The mean age of the sample was 12.73 years (SD $=.54$, range 11-15 years). Children were primarily Caucasian (>90\%). Due to school constraints, information about the socioeconomic status and family structure of the children was not available. All children completed the questionnaires in their classrooms with the teacher and a research assistant always present to ensure independent and confidential responding and to provide assistance if necessary. Children and parents were informed about the aim and content of the study. None of the participants refused to participate, yielding a response rate of $100 \%$. 


\section{Questionnaires}

Inspired by the work of Gest (1997), Muris et al. (1999) developed the Behavioral Inhibition Scale (BIS), a brief self-report questionnaire for assessing behavioral inhibition. The BIS consists of 4 items referring to shyness, communication, fearfulness, and smiling (e.g., "I feel nervous when I have to talk to an unfamiliar person"). Each item is scored on a 4-point Likert scale: 1 means never, 2 sometimes, 3 often, and 4 always. After recoding the positive items, scores are summed to yield a total BIS score, ranging from 4 (not apprehensive, not shy and very sociable when meeting an unfamiliar person) to 16 (very apprehensive and shy and not capable of initiating social interaction with an unfamiliar person). Previous research has yielded support for the reliability and validity of the BIS. To begin with, in a recent study by Van Brakel, Muris, and Bögels (2004), the relation between the BIS and observable manifestations of behavioral inhibition was examined. Moderate but significant relations were found between parent- and teacher-reported behavioral inhibition of the child as measured by the BIS and the observational index of this temperamental trait, thus providing evidence for the validity of the scale. Further, an investigation by Muris et al. (2003a) showed that self-report BIS scores had acceptable correlations with parent ratings on the BIS. Finally, the reliability of the BIS appears good, with Cronbach's alphas well above .80 and a test-retest correlation of .77 over a 2-year period (see Van Brakel \& Muris, in press).

The Attachment Questionnaire for Children (AQ-C) consists of three descriptions concerning children's feelings about and perceptions of their relationships with other children: (1) "I find it easy to become close friends with other children. I trust them and I am comfortable depending on them. I do not worry about being abandoned or about another child getting too close friends with me." (secure attachment); (2) "I am uncomfortable to be close friends with other children. I find it difficult to trust them completely, difficult to depend on them. I get nervous when another child wants to become close friends with me. Friends often come more close to me than I want them to." (avoidant attachment); and (3) "I often find that other children do not want to get as close as I would like them to be. I am often worried that my best friend doesn't really like me and wants to end our friendship. I prefer to do everything together with my best friend. However, this desire sometimes scares other children away." (ambivalent attachment). Children are provided with these descriptions and instructed to choose the description that applies best to them. In this way, they classify themselves as either securely, avoidantly, or ambivalently attached. In the present study, the AQ-C was dichotomized after the children had chosen one of the three descriptions. That is, secure attachment was recoded as 0 , whereas 
avoidant and ambivalent attachment were both recoded as 1 . In a study by Muris et al. (2001a), the connection between the AQ-C and a concurrent measure of attachment, the Inventory of Parent and Peer Attachment (IPPA; Armsden \& Greenberg, 1987), was examined. Results showed that adolescents who classified themselves as securely attached on the AQ-C displayed a higher quality of attachment to both parents and peers than adolescents who classified themselves as insecurely (i.e., avoidantly or ambivalently) attached on the AQ-C. Clearly, this finding supports the validity of the AQ-C.

Modified EMBU-C (Child version of the Egna Minnen Beträffende Uppfostran, which is Swedish for My memories of upbringing; Castro, Toro, Van der Ende, \& Arrindell, 1993; Muris, Bosma, Meesters, \& Schouten, 1998). The EMBU-C originally is a 41 -item questionnaire measuring four types of parental rearing: emotional warmth, rejection, control, and favoring subject. Grüner et al. (1999) modified the EMBU-C in three ways. First of all, new items were added in an attempt to measure children's perceptions of their parents' anxious rearing behaviors. Second, all items referring to children's brothers and sisters (i.e., the favoring subject subscale and two additional items) were removed because not all children have brothers and sisters. Third, for each type of parental rearing, the number of items was reduced to 10. As a result, the modified EMBU-C consists of 40 items that can be allocated to four types of parental rearing: emotional warmth, rejection, control, and anxious rearing. For each EMBU-C item, children first assess father's rearing behavior and then mother's rearing behavior, using 4-point Likert-scales ( $1=$ No, never, 2 = Yes, but seldom, $3=$ Yes, often, $4=$ Yes, most of the time). The psychometrics of the modified EMBU-C were tested in a large sample of children and adolescents $(N=1702)$. Results showed that the scale has a clear-cut 4 -factor structure, which is in correspondence with the hypothesized subscales. Furthermore EMBU-C scales were reliable in terms of internal consistency and test-retest stability (Muris, Meesters, \& Van Brakel, 2003b). In the current study only two EMBU-C subscales were used, control (e.g., "When you come home, you have to tell your parents what you have been doing") and anxious rearing (e.g., "Your parents are scared when you do something on your own"), as these seem most relevant in relation to anxiety symptoms (Muris et al., 2003b).

The Screen for Child Anxiety Related Emotional Disorders (SCARED) is a 41 item self-report questionnaire for measuring symptoms of panic, social phobia, separation anxiety, generalized anxiety, and school phobia (Birmaher, Brent, Chiapetta et al., 1999). Children have to indicate how frequently they experience each symptom (e.g., "I worry about things working out for me", "When I am 
frightened, my heart beats fast") on a 3-point scale: $0=$ almost never, $1=$ sometimes, and 2 = often. In the present study, the SCARED total anxiety score was used, which can be obtained by summing across relevant items.' Previous research has demonstrated that the SCARED has good internal consistency (e.g., Muris, Schmidt, \& Merckelbach, 2000c; Birmaher, Khetarpal, Brent et al., 1997), test-retest reliability, and discriminant validity (e.g., Birmaher et al., 1997, 1999).

\section{RESULTS}

\section{General findings}

Before addressing the main research questions of this current study, some general remarks should be made. First, all questionnaires were reliable in terms of internal consistency, with Cronbach's alpha being .77 for the BIS, .65 for EMBU-C control, .77 for EMBU-C anxious rearing, and .91 for the SCARED. Second, $t$-tests revealed that girls, in comparison to boys, scored significantly higher on anxiety symptoms (SCARED) $[t(641)=5.34, p<.001]$ and behavioral inhibition (BIS) $[t(642)=3.23$, $p<.011$. Moreover, girls rated themselves more often as being insecure attached than boys $\left[X^{2}(1)=5.07, p<.05\right]$. Thus, girls were found to be generally more distressed than boys, which is in keeping with what has been reported in the literature (e.g., Craske, 1997). Third, no significant associations between age and any of the measures emerged. Fourth, as rearing behaviors of mothers and fathers were significantly related to each other [rs were .72, $p<.001$ for control and $.76, p<$ .001 for anxious rearing], scores of mother and father were summed, and these total control and anxious rearing scores were used in further analyses.

\section{Correlations among risk factors}

There were small to modest but significant intercorrelations among various risk factors (Table 1). More specifically, behavioral inhibition correlated 32 with insecure attachment, .11 with control, and .14 with anxious rearing. Insecure attachment

\footnotetext{
' Analyses examining main and interactive effects of various vulnerability factors on SCARED anxiety subscales generally yielded comparable results. That is, in most equations, significant main effects of behavioral inhibition, attachment, and/or parental rearing were found, whereas interactive effects were generally weak and non-significant. Total percentages of explained variance varied between $10.0 \%$ (school phobia) and $57.3 \%$ (social phobia).
} 
correlated .14 and .10 with respectively control and anxious rearing, whereas a correlation of .52 was found between both types of rearing behaviors. Altogether, these findings indicate that although these risk factors share some common variance, they seem to represent a set of relatively independent constructs.

Table 1: The number (percentage) of children who classified themselves as insecurely attached on the AQ-C, mean scores (standard deviations) for BIS, EMBU-C scales, and SCARED, and Pearson correlations among all variables

\begin{tabular}{|c|c|c|c|c|c|c|c|}
\hline & $\begin{array}{l}\text { Total } \\
\text { group }\end{array}$ & Boys & Girls & (7) & (2) & (3) & (4) \\
\hline $\begin{array}{l}\text { (1) AQ-C } \\
\text { Insecurely } \\
\text { attached }\end{array}$ & $\begin{array}{c}100 \\
(15.5)\end{array}$ & $\begin{array}{c}42 \\
(12.5)_{a}\end{array}$ & $\begin{array}{c}58.0 \\
(19.0)_{b}\end{array}$ & & & & \\
\hline (2) BIS & $\begin{array}{l}9.4 \\
(2.2)\end{array}$ & $\begin{array}{l}9.1 \\
(2.1)_{a}\end{array}$ & $\begin{array}{l}9.6 \\
(2.2)_{b}\end{array}$ & $.33^{* *}$ & & & \\
\hline $\begin{array}{l}\text { (3) EMBU-C } \\
\text { Control }\end{array}$ & $\begin{array}{l}20.0 \\
(3.4)\end{array}$ & $\begin{array}{l}20.2 \\
(3.4)_{a}\end{array}$ & $\begin{array}{l}19.8 \\
(3.4)_{a}\end{array}$ & $.13^{* *}$ & $.10^{* *}$ & & \\
\hline $\begin{array}{l}\text { (4) EMBU-C } \\
\text { Anxious rearing }\end{array}$ & $\begin{array}{l}20.5 \\
(4.2)\end{array}$ & $\begin{array}{l}20.5 \\
(4.2)_{a}\end{array}$ & $\begin{array}{l}20.6 \\
(4.2)_{a}\end{array}$ & $.10^{*}$ & $.74^{* *}$ & $.52^{* *}$ & \\
\hline (5) SCARED & $\begin{array}{l}16.0 \\
(10.2)\end{array}$ & $\begin{array}{l}14.0 \\
(9.0)_{a}\end{array}$ & $\begin{array}{l}18.0 \\
(11.0)_{b}\end{array}$ & $.40^{* *}$ & $.52^{* *}$ & $.21^{* *}$ & $.30 * *$ \\
\hline \multicolumn{8}{|c|}{$\begin{array}{l}\text { Note. } N=644 \text { (337 boys and } 307 \text { girls). AQ-C = Attachment Questionnaire for Children, BIS } \\
=\text { Behavioral Inhibition Questionnaire, EMBU-C }=\text { Child version of the Egna Minnen } \\
\text { Beträffende Uppfostran (My memories of upbringing), SCARED = Screen for Child Anxiety ana } \\
\text { Related Emotional Disorders. }{ }^{*} p<.05,{ }^{* *} p<.01 \text {. Means with different subscripts indicate a } \\
\text { significant gender difference }(p<.05) \text {. }\end{array}$} \\
\hline
\end{tabular}

\section{Relationship with anxiety symptoms}

Table 1 also shows Pearson correlations between behavioral inhibition, attachment, and rearing behaviors, on the one hand, and anxiety symptoms, on the other hand. Results indicated that there were modest but significant positive correlations between behavioral inhibition, insecure attachment, control, and anxious rearing, on the one hand, and anxiety scores on the other hand. That is, higher levels of behavioral inhibition, insecure attachment, parental control and anxious rearing were associated with higher levels of anxiety symptoms. As girls displayed higher levels of anxiety symptoms and behavioral inhibition, and were more frequently insecurely attached than boys, correlations were also computed for boys and girls separately. For both genders, results were highly similar as those obtained for the total group. 
Additive and interactive effects of risk factors on anxiety.

Stepwise hierarchical regression analyses were carried out in order to examine the additive and interactive effects of various risk factors to adolescents' anxiety symptoms. In these analyses, scores on BIS, AQ-C, and EMBU-C were the predictor variables, whereas the total score on the SCARED served as the dependent variable. The following strategy was used for entering various predictor variables. On the first two steps, the main effects of the child factors of insecure attachment and behavioral inhibition, and their interaction were examined. The following step included the environmental factor of parental rearing, and the final steps investigated interaction effects of child and environmental factors.

Table 2: Main results of the regression analyses with behavioral inhibition, attachment quality, and parental control being predictors and anxiety disorder symptoms as the dependent variable

\begin{tabular}{|c|c|c|c|c|}
\hline & $B$ & SE B & $\beta$ & $\Delta R^{2}$ \\
\hline Step 1 & & & & $.33^{* * *}$ \\
\hline $\begin{array}{l}\text { Behavioral Inhibition } \\
\text { Attachment }\end{array}$ & $\begin{array}{l}4.41 \\
7.36\end{array}$ & $\begin{array}{l}0.35 \\
0.96\end{array}$ & $\begin{array}{l}.43^{* * *} \\
.26^{* * *}\end{array}$ & \\
\hline Step 2 & & & & $.00^{*}$ \\
\hline Behavioral Inhibition $\times$ Attachment & 1.83 & 0.90 & $.09^{*}$ & \\
\hline Step 3 & & & & $.02^{* * *}$ \\
\hline Control & 1.31 & 0.33 & $.13^{* * *}$ & \\
\hline Step 4 & & & & $.00^{*}$ \\
\hline $\begin{array}{l}\text { Behavioral Inhibition } \times \text { Control } \\
\text { Attachment } x \text { Control }\end{array}$ & $\begin{array}{l}0.28 \\
-0.92\end{array}$ & $\begin{array}{l}0.34 \\
0.87\end{array}$ & $\begin{array}{l}.03 \\
-.04\end{array}$ & \\
\hline Step 5 & & & & $.01^{* *}$ \\
\hline $\begin{array}{l}\text { Behavioral Inhibition } \times \text { Attachment } \\
\times \text { Control }\end{array}$ & 2.26 & 0.83 & $.12^{* *}$ & \\
\hline
\end{tabular}

Note. $N=644 .{ }^{*} p<.05,{ }^{* *} p<.01,{ }^{* * *} p<.001$.

Table 2 shows the main results of the regression analyses with behavioral inhibition, insecure attachment, and parental control being the predictors and anxiety disorder symptoms as the dependent variable. The results indicate that behavioral inhibition (beta $=.43 ; p<.001$ ), insecure attachment (beta $=.26 ; p<.001$ ), and control (beta $=.13 ; p<.001$ ) each accounted for a small but unique proportion of the variance, which indicates that all these factors had additive predictive value for 
adolescents' anxiety. Two significant interaction effects were found. To begin with, behavioral inhibition and insecure attachment had an interactive effect on anxiety (beta $=.09, \mathrm{p}<.05$ ). Although this effect was small (as it accounted for less than $1 \%$ of the variance), the pattern of results was as expected: that is, children who defined themselves as high on behavioral inhibition and insecurely attached displayed the highest levels of anxiety symptoms, whereas children who classified themselves as low on inhibition and securely attached exhibited the lowest levels of anxiety symptoms. Further, a higher-order effect was found for the interaction between behavioral inhibition, attachment quality, and control (beta $=.12 ; p<$ $.01)$. Figure 1 visualizes this higher-order interaction. As expected, children who define themselves as high on behavioral inhibition and insecurely attached showed higher levels of anxiety symptoms. The role of parental control, however, was less consistent. In some children (i.e., uninhibited/securely attached and inhibited/insecurely attached), high levels of this rearing factor were associated with higher anxiety levels, whereas in other children (i.e., inhibited/securely attached) high control was related to lower anxiety levels.

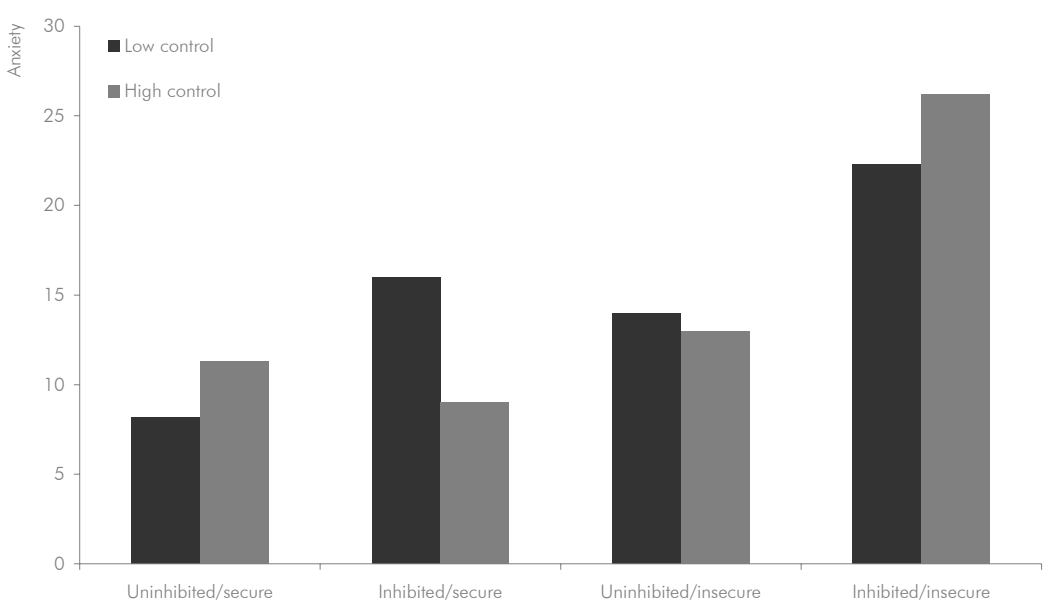

Figure 1: Visualization of the higher-order interaction between behavioral inhibition (BIS), attachment (AQ-C), and parental control (EMBU-C) on anxiety disorders symptoms

Note. $N=644$. 
Table 3: Main results of the regression analyses with behavioral inhibition, attachment quality, and anxious rearing being predictors and anxiety disorder symptoms as the dependent variable

\begin{tabular}{|c|c|c|c|c|}
\hline & $B$ & SE B & $\beta$ & $\Delta R^{2}$ \\
\hline Step 1 & & & & $.33^{* * *}$ \\
\hline Behavioral Inhibition & 4.41 & 0.35 & $.43^{* * *}$ & \\
\hline Attachment & 7.36 & 0.96 & $.26^{* * *}$ & \\
\hline Step 2 & & & & $.00^{*}$ \\
\hline Behavioral Inhibition x Attachment & 1.83 & 0.90 & $.09^{*}$ & \\
\hline Step 3 & & & & $.05^{* * *}$ \\
\hline Anxious Rearing & 2.21 & 0.32 & $.22^{* * *}$ & \\
\hline Step 4 & & & & .00 \\
\hline $\begin{array}{l}\text { Behavioral inhibition x Anxious } \\
\text { Rearing }\end{array}$ & 0.71 & 0.36 & .07 & \\
\hline Attachment $x$ Anxious Rearing & -0.19 & 0.86 & -.01 & \\
\hline Step 5 & & & & .00 \\
\hline $\begin{array}{l}\text { Behavioral Inhibition x Attachment } \\
x \text { Anxious Rearing }\end{array}$ & 0.92 & 0.83 & .05 & \\
\hline
\end{tabular}

Table 3 shows the main results of the regression analysis with behavioral inhibition, attachment quality, and anxious rearing as predictors and anxiety disorder symptoms being the dependent variable. The results indicate that behavioral inhibition (beta $=.43 ; p<.001$ ), insecure attachment (beta $=.26 ; p<.001)$, and anxious rearing (beta $=.22 ; p<.001$ ) each accounted for a small but unique proportion of the variance. Besides the significant interaction of behavioral inhibition and attachment (see supra), no interaction effects were found.

Separate analyses for boys and girls essentially yielded similar results. In all analyses, significant main effects of behavioral inhibition, attachment, and parental rearing were found (all betas between .14 and .48, all ps $<.01$ ). The interaction effects that were found when analysing the total sample disappeared, which underlines that these effects were not very robust. 


\section{DISCUSSION}

The present study was a first attempt to examine the reciprocal connections among temperament, attachment, and parental rearing style, and their additive and interactive contributions to anxiety symptoms. The main results of the study can be catalogued as follows. First, there were small to modest positive correlations among behavioral inhibition, insecure attachment, and the hypothesized anxiety-promoting rearing behaviors of control and anxious rearing, indicating that these child and environmental risk factors were to some extent related to each other. Second, behavioral inhibition, insecure attachment, and parental control and anxious rearing were positively associated with higher levels of anxiety symptoms. Finally, behavioral inhibition, insecure attachment, and parental rearing each accounted for a unique proportion in the variance of anxiety disorders symptoms.

The results of the current study seem to indicate that behavioral inhibition, insecure attachment, and parental rearing predominantly have additive effects on the development of anxiety. Few interaction effects among these vulnerability factors were found, and it should be emphasized that such effects were rather small. For example, the interaction effect of attachment and behavioral inhibition was theoretically meaningful (with the combination of insecure attachment and high levels of inhibition yielding the highest anxiety scores), but accounted for less than $1 \%$ of the variance. The higher-order interaction effect of behavioral inhibition, insecure attachment, and control on anxiety symptoms was somewhat larger. This effect was due to the fact that, in some children (i.e., uninhibited/securely attached and inhibited/insecurely attached), high levels of this rearing factor were associated with higher anxiety levels, whereas in other children (i.e., inhibited/securely attached), high control was related to lower anxiety levels. These divergent effects of parental control on anxiety can be explained when one adopts the notion that this rearing factor has multiple faces. On the one hand (as mentioned in the introduction), control may be associated with extreme strictness, which has the negative consequence of reducing the development of autonomy. On the other hand, control has the positive consequence of structuring the child's environment. When looking at our data, it can be suggested that on the condition that inhibited children have parents who are sensitive and responsive (in such a way that caregiver and child are securely attached), a highly controlling parenting style has a positive influence on the anxiety symptoms of these children. That is, in this group of children, highly controlling parents offer just the structure these children need to help them navigate through their daily lives, and hence reduce anxiety. However, when children do not 
need such assistance (i.e., the uninhibited/secure group) or when children do need guidance but their parents cannot provide it (i.e., the inhibited/insecure group), control may manifest itself in its negative overprotective way and thus enhance anxiety.

It should be acknowledged that the current study suffers from several shortcomings. First of all, the study solely relied on adolescents' self-report. Inclusion of parent versions of various questionnaires would have provided important crossvalidational information on the role of behavioral inhibition, attachment, and rearing behaviors in the development of childhood anxiety. Especially, knowing that children who are significantly anxious often have cognitive distortions that may contribute to perceiving others (e.g. parents) in an overly threatening/negative way. For this reason, multiple informants or observational measures should be used in future studies. Second, the participants in this study were still relatively young. As it is clear that some anxiety symptoms (i.e., social phobia, generalized anxiety, panic disorder) become more clearly manifest in middle or late adolescence (e.g., Costello \& Angold, 1995), it remains unclear to what extent the results can be generalized to older children. A third shortcoming has to do with the scales that were employed to measure attachment. Previous research has shown that children of this age have a tendency to endorse the positively toned, secure attachment item rather than the more negatively toned descriptions of avoidant and ambivalent attachment. Thus, reliance on the AQ-C to assess children and adolescents' attachment style may lead to an underreporting of insecure attachment types (Muris et al., 2000a, 2001a). Clearly, a study examining the link between self-reported attachment and observational and narrative measures of this construct could clarify this issue. In a similar vein, one could question the validity of the BIS and simply qualify it as a measure of social anxiety. Note, however, that the BIS was developed closely following the definition of this temperamental construct (see Gest, 1997) and previous research has demonstrated that the overlap with social anxiety is at best modest (Van Brakel et al., 2004). A final point of critique pertains to the fact that we only assessed a restricted number of risk factors. For example, one important vulnerability factor that was not included is parental anxiety. Many findings support the notion that relationships between anxious parents and their children are characterized by different factors than those between normal parents and their children (Turner et al., 2003). Thus, data on parental anxiety would have enabled us to differentiate between children with a family history of anxiety and those without such history. In this way, we could have mapped the differences of these families with 
respect to attachment relationships, behavioral inhibition, and parental rearing behaviors.

Despite these limitations, the current data provide further support for the notion that various potential predisposing factors have additive and, to some extent, interactive effects on anxiety. Of course, longitudinal studies, preferably including clinically referred youths, are needed to shed light on the direction of relations between behavioral inhibition, attachment, parental rearing behavior, and other risk and protective factors, on the one hand, and childhood anxiety problems, on the other hand. On this very moment, such longitudinal project on the development of anxiety in children is carried out (Van Brakel, Muris \& Bögels, 2001), and thus more convincing evidence on the additive and interactive effects of various risk factors is underway. 



\section{CHAPTER 6}

Behavioral inhibition as a risk factor for the development of childhood anxiety disorders: A longitudinal study 


\section{ABSTRACT}

This longitudinal study was the first attempt to examine the additive and interactive effects of behavioral inhibition and a wide range of other vulnerability factors in the development of anxiety problems in youths. A sample of 261 children, aged 5 to 8 years, 124 who had been identified as behaviorally inhibited and 137 control children, were followed during a 3-year period. Assessments took place on three occasions to measure children's level of behavioral inhibition, anxiety disorder symptoms, other psychopathological symptoms, and a number of other vulnerability factors such as insecure attachment, negative parenting styles, adverse life events, and parental anxiety. Results obtained with Structural Equation Modeling indicated that behavioral inhibition primarily acted as a specific risk factor for the development of social anxiety symptoms. Furthermore, the comprehensive longitudinal model showed additive as well as interactive effects for various vulnerability factors on the development of anxiety symptoms. That is, main effects of anxious rearing and parental trait anxiety were found, whereas behavioral inhibition and attachment had an interactive effect on anxiety symptomatology. Moreover, behavioral inhibition itself was also influenced by some of the vulnerability factors. But, no support was found for the hypothesis that behaviorally inhibited children who experienced adverse life events would be particularly prone to display an increase of anxious symptoms. These results provide support for dynamic, multifactorial models for the etiology of child anxiety problems. 


\section{BEHAVIORAL INHIBITION AS A RISK FACTOR FOR THE DEVELOPMENT OF ANXIETY DISORDERS IN CHILDREN: A LONGITUDINAL STUDY}

Behavioral inhibition is a temperamental trait characterized by a relatively consistent pattern of behavioral and emotional responses to unfamiliar people and novel stimuli and situations. That is, inhibited children typically respond with restraint, caution, and withdrawal to novel objects and situations, and they are usually timid, fearful, and shy with unfamiliar people (Kagan, Reznick, \& Snidman, 1988; Kagan, 1994). Behavioral inhibition shows moderate to good levels of continuity during childhood and is to a certain extent also associated with aspects of adult personality (for a review of the research on lifetime continuity in behavioral inhibition, see Fox, Henderson, Marshall, Nichols, \& Ghera, 2005). During the past 15 years, a number of studies have shown that there is an increased prevalence of anxiety disorders among behaviorally inhibited children (e.g., Biederman, Rosenbaum, Hirschfeld et al., 1990; Hirshfeld-Becker, Biederman, \& Rosenbaum, 2004). Noteworthy in this regard is the 3-year longitudinal study by Biederman and colleagues (1993) who found that children initially identified as behaviorally inhibited were subsequently more likely to develop anxiety disorders as compared to control children (i.e., children who at study onset were not classified as behaviorally inhibited). It should be noted that in particular children with stable behavioral inhibition ran an increased risk for developing pathological anxiety (see also Hirshfeld-Becker, Rosenbaum, Biederman et al., 1992).

While there is clear evidence indicating that behavioral inhibition is an important risk factor for the development of pathological anxiety in youths, it should also be noted that not every child with an inhibited temperament develops an anxiety disorder (see for instance Biederman et al., 1990), and so the question arises under what conditions behaviorally inhibited children develop anxiety disorders. Further, not every anxious child has a behaviorally inhibited temperament (Turner, Beidel, \& Wolff, 1996). As with other types of psychopathology, it is generally assumed that anxiety disorders are the result of multiple variables and influences (Vasey \& Dadds, 2001). Therefore, when studying the role of behavioral inhibition in childhood anxiety disorders, one should also consider other vulnerability factors so that it becomes possible to study the unique and interactive effects of this temperamental trait. In the literature, a number of other factors have been identified that may play a role in the formation of anxiety problems, which operate in conjunction or interaction 
with behavioral inhibition. These include an insecure parent-child attachment, negative parenting styles, parental anxiety, and adverse life events.

\section{Insecure attachment}

In their longitudinal study, Warren and colleagues (1997) found a specific link between insecure (in particular, ambivalent) attachment measured in infancy and anxiety disorders that were assessed some 16 years later. A couple of studies have examined insecure attachment in interaction with behavioral inhibition (e.g., Calkins \& Fox, 1992; Mannasis, Bradley, Goldberg, Hood, \& Swinson, 1995; Muris, Meesters, Merckelbach, \& Hülsenbeck, 2000a; Muris \& Meesters, 2002, ShamirEssakow, Ungerer, \& Rapee, 2005). Altogether, the results of these studies have shown that children who combine a behaviorally inhibited temperament with an insecure attachment status display the highest levels of anxiety disorders symptoms, which of course indicates that these two vulnerability factors have a cumulative effect on the development of anxiety pathology in youths.

\section{Negative parenting styles}

Two parenting dimensions that have been consistently associated with the development of anxiety in children and adolescents are control and anxious rearing (e.g., Wood, McLeod, Sigman, Hwang, \& Chu, 2003). Control can best be described as parental behaviors aimed at guiding children during their daily activities. These parental behaviors often have the effect of directing the child and reducing the development of autonomy (Rapee, 1997). Anxious rearing pertains to the explicit encouragement of anxious cognitions and avoidance behaviors in children (e.g., Barret, Rapee, Dadds, \& Ryan, 1996; Grüner, Muris, \& Merckelbach, 1999). Several studies have yielded evidence for the proposed relationship between controlling and anxious rearing behaviors and anxiety disorder symptoms, some of them relying on direct observation of parent-child interactions (Hudson \& Rapee, 2001; Whaley, Pinto, \& Sigman, 1999) and others making use of questionnaires that intend to measure children's perceptions of parental rearing behaviours (Grüner et al., 1999; Muris et al., 2000a; Muris, Meesters, \& Van Brakel, 2003b). Controlling and anxious parenting have been found to play a unique role in the development of childhood anxiety symptoms when examined together with behavioral inhibition (Van Brakel, Muris, Bögels, \& Thomassen, 2006), and there are also studies indicating that parenting interacts with an inhibited temperament (Rubin, Hastings, Stewart, Henderson, \& Chen, 1997; Rubin, Nelson, Hastings, \& Asendorpf, 1999). 


\section{Adverse life events}

Clear associations exist between adverse life events and psychiatric disorders in children and adolescents and these also include anxiety disorders (Tiet, Bird, Hoven et al., 2001). It seems plausible to assume that behaviorally inhibited children are particularly prone to develop anxiety problems when confronted with negative life events. Brozina and Abela (2006) tested this idea and examined the relationship between behavioral inhibition and anxiety symptoms within a diathesis-stress framework. The results of this study indicated that only behaviorally inhibited children who experienced high levels of stressful events (e.g., fighting parents, being teased at school, being punished) displayed an increase of anxiety symptoms during a 6weeks period. In contrast, behaviorally inhibited children who did not experience such events exhibited a decrease in anxiety symptoms.

\section{Parental anxiety}

Research has shown that children of parents with anxiety disorders run a greater risk for developing anxiety disorders themselves (Biederman, Rosenbaum, Bolduc, Faraone, \& Hirshfeld, 1991). Furthermore, Rosenbaum, Biederman, Gersen et al. (1988) reported that a substantial proportion of the children of parents with clinically significant anxiety disorders were behaviorally inhibited. Finally and most importantly, there is also evidence to demonstrate that children with a behaviorally inhibited temperament who also have parents suffering from an anxiety disorder are most vulnerable to develop anxiety disorders (e.g., Rosenbaum, Biederman, Bolduc et al., 1992).

\section{Behavioral inhibition: A specific or a general risk factor?}

Many studies suggest that behavioral inhibition is associated with the development of a wide range of anxiety-related symptoms and disorders (Biederman et al., 1993; Gest, 1997; Muris et al., 1999, 2001, 2003a; Reznick et al., 1992; ShamirEssakow et al., 2005; Turner et al., 1996), but there are also several studies that indicate that behavioral inhibition is a more specific risk factor and is only involved in the pathogenesis of social anxiety. For example, Townsley-Stemberger, Turner, Beidel, and Calhoun (1995) noted that social phobic patients reported significantly higher levels of shyness (which seems to be a clear marker of behavioral inhibition) in childhood than did control participants. In line with these findings, Mick and Telch (1998) asked students with high symptom levels of social anxiety or generalized anxiety to retrospectively report their level of behavioral inhibition during childhood. These researchers found that behavioral inhibition in childhood was strongly 
associated with symptoms of social anxiety in adulthood, but not with symptoms of generalized anxiety. Hayward, Killen, Kraemer, and Taylor (1998) showed that adolescents $(N=2242)$ who retrospectively reported that they had been behaviorally inhibited in childhood were four to five times more likely to suffer from a social phobia than adolescents who reported that they had not been behaviorally inhibited in childhood. Recently, Gladstone and colleagues (2005) have also demonstrated that a behaviorally inhibited temperament in childhood may be a specific vulnerability factor to social anxiety rather than to anxiety in general.

Adolescents' self-reports of behavioral inhibition are not only positively correlated with anxiety, but also with depression symptoms (Muris et al., 1999, $2001,2003 a$ ). One explanation for this association is that behavioral inhibition acts as a common vulnerability factor to both anxiety and depression. Another explanation could be that the association between behavioral inhibition and depression is a statistical artifact of the strong associations between anxiety and both behavioral inhibition and depression (Brozina \& Abela, 2006). Gladstone and Parker (2006) obtained evidence to suggest that the presence of a behaviorally inhibited temperament may pose a developmental risk for lifetime depression. However, it is important to note that the relationship between behavioral inhibition and depression was dependent on the presence of social anxiety. That is, only behaviorally inhibited children who had developed social anxiety were prone to develop a depression (see also Muris et al., 2003a). Nevertheless, other studies can be found in the literature indicating that behavioral inhibition is clearly associated with depression (HirshfeldBecker, Biederman, Calltharp, Rosenbaum, Faraone, \& Rosenbaum, 2003; Gullone, Ollendick, \& King, 2006; Jaffee, Moffitt, Caspi, Fombonne, \& Martin, 2002; Shatz, 2005). Taken together, although it has been proposed that behavioral inhibition is associated with the development of a wide range of anxiety-related symptoms and disorders, there is also evidence indicating that this temperamental trait acts as a more specific risk factor for social anxiety. In addition, more research is required to examine whether behavioral inhibition is specifically linked to anxiety symptomatology or whether this temperamental trait also plays a role in the etiology of depression or even other psychological disorders in youths.

So far, research has demonstrated that behavioral inhibition, and other vulnerability factors like insecure attachment, negative parenting styles, adverse life events, and parental anxiety contribute to the development of anxiety disorders in youths. Only a few studies can be found that have investigated whether combinations of these factors yield an increased risk for anxiety problems (e.g., Calkins \& Fox, 1992; Van Brakel et al., 2006; Brozina \& Abela, 2006), but no study can be found 
that examined the additive and interactive effects of behavioral inhibition and a wide range of other vulnerability factors in the development of pathological anxiety in youths. With this issue in mind, the present longitudinal research project was set-up. A group of 261 children, 124 of whom had been classified as high on behavioral inhibition and 137 control children, were followed during a 3-year period. Each year, an assessment took place to measure children's level of behavioral inhibition, anxiety disorder symptoms, other psychopathological symptoms, and a number of other vulnerability factors such as insecure attachment, negative parenting, adverse life events, and parental anxiety. In this way, it became possible to study (1) the unique and interactive effect of behavioral inhibition on the development of anxiety symptoms, and (2) whether behavioral inhibition plays a role in the development of anxiety problems in general, social anxiety, or a broad range of psychopathological symptoms (e.g., depression).

It was hypothesized that behavioral inhibition would be predictive of anxiety symptoms, especially social anxiety symptoms, over the 3-year period. Consistent with a diathesis-stress framework (see Brozina \& Abela, 2006), it was expected that behaviorally inhibited children who experienced high levels of stress as a result of adverse life events would be particularly prone to display an increase of anxious symptoms. Further, insecure attachment, an anxious and overprotective parenting style, and parental anxiety were all expected to play a unique role in the development of children's anxious symptoms, and it was explored to what extent these vulnerability factors interacted with behavioral inhibition.

\section{METHOD}

\section{Participants and procedure}

The parents of 2547 children of 40 normal primary schools in the southern part of The Netherlands were approached by mail. By means of a letter, parents received information about this longitudinal research project, and they were asked to give their consent about their child's participation in the study. In addition, parents were invited to complete the Behavioral Inhibiton Questionnaire (BIQ; see below) and to return materials to the researchers in a sealed envelop. One-thousand-and-onehundred-seventy-six parents (46.2\%) responded positively to this invitation and allowed their child to participate in the study. Two-hundred-and-twenty-seven of these children were identified as behaviorally inhibited by means of the BIQ scores as provided by their parents, and 184 control children were selected because they scored in the normal range of the BIQ. The parents of these 411 children were 
approached by phone to explain the procedure of the longitudinal study once again. After this phone call, 261 parent couples were convinced about their commitment to the study for a couple of years, which resulted in a final sample of 124 behaviorally inhibited children and 137 control children. Both groups were comparable in terms of gender distribution [inhibited group: 58 boys and 66 girls versus control group: 57 boys and 80 girls; $X^{2}(1)=.55, p=.46$ ] and age [in both groups: age ranged between 5 and 8 , with a mean of 6.6 years; $t(258)=.55, p=.58$ ]. The percentages of families with a low, middle, or high socio-economic background (classified by means of the occupational levels of both parents, employing the guidelines provided by the Dutch Central Bureau of Statistics) were 15\%,20\%, and $65 \%$, respectively. The majority of the children and their parents were Caucasian (97\%). The remaining families (3\%) had a Mediterranean, Asian, or North-African background. About $90 \%$ of the children came from two-parent families. During the course of the study, only 14 children dropped out. In Table 1, various assessment occasions of this longitudinal project are shown as well as the specific assessment instruments that were administered on each point-in-time.

Table 1: Assessment occasions of this longitudinal project as well as the specific instruments that were administered on each point-in-time

\begin{tabular}{llll}
\hline Screening & $\begin{array}{l}\text { Occasion I } \\
6 \text { months later }\end{array}$ & $\begin{array}{l}\text { Occasion II } \\
\text { 1 year later }\end{array}$ & $\begin{array}{l}\text { Occasion III } \\
\text { 2 years later }\end{array}$ \\
\hline Behavioral inhibition: & Behavioral inhibition: & Behavioral inhibition: & Anxiety symptoms \\
BIQ & BIQ & Parental rearing: & \\
& Parental rearing: & EMBU \\
EMBU & Trait anxiety father & \\
Trait anxiety father & and mother: STAl & \\
and mother: STAI & Psychopathology & Psychopathology \\
children: DOMINIC & children: DOMINIC & \\
& Attachment: ASQ & \\
& Life events during \\
& past year: LES & \\
&
\end{tabular}

Note. $B I Q=$ Behavioral Inhibition Questionnaire; EMBU = Egna Minnen Beträffende Uppfostran, which is Swedish for My memories of upbringing; STAl = State Trait Anxiety Inventory; $A S Q=$ Attachment Style Questionnaire; LES = Life Experiences Survey; SCARED = Screen for Child Anxiety Related Emotional Disorders; DOMINIC = questionnaire to assess symptoms of the 7 prevalent DSM-III-R disorders in children. 
On occasion I, children were visited by a research assistant at their schools. They were given a structured interview for measuring psychopathological symptoms. Following this, children participated in a behavioral observation procedure: children were confronted with novel stimuli and unknown persons, and their behavior was coded in terms of behavioral inhibition characteristics (for a detailed description and the results obtained with this procedure: see Van Brakel, Muris, \& Bögels, 2004). Parents were sent an envelope containing questionnaires to assess their child's level of behavioral inhibition and psychopathological symptoms, their own rearing behaviors and level of trait anxiety. On occasion II, one year later, children were revisited at school by a research assistant who administered a structured interview to measure psychopathological symptoms. Again, parents were sent questionnaires for measuring behavioral inhibition and psychopathological symptoms of their child, parent-child attachment quality, rearing behaviors, and trait anxiety, and the number and impact of life events that had occurred during the past year. On occasion III, some two years later, parents as well as children were asked to complete the SCARED, a questionnaire for measuring children's anxiety symptoms in terms of the DSM-IV. A detailed description of all questionnaires and interviews will follow in the next section.

\section{Measures}

Inspired by the work of Gest (1997), Muris et al. (1999) developed the Behavioral Inhibition Questionnaire (BIQ). The $B I Q$ is a brief questionnaire for assessing behavioral inhibition, which consists of two parts: (1) the Behavioral Inhibition Scale (BIS) and (2) the Behavioral Inhibition (BI) categories. The parent version of the BIS contains 8 items referring to children's shyness, fearfulness, and communication with unfamiliar people (e.g., "My child talks easily to an unfamiliar child", "My child feels nervous when he/she has to talk to an unfamiliar adult"; see also Van Brakel et al., 2004). Each item is scored on a 4-point Likert scale with $1=$ never, $2=$ sometimes, $3=$ often, and 4 = always. After recoding the positive items, scores are summed to yield a total BIS score, ranging from 8 (not apprehensive, not shy and very sociable when meeting unfamiliar persons) to 32 (very apprehensive and shy and not capable of initiating social interaction with unfamiliar persons). The $\mathrm{Bl}$ categories are three descriptions concerning children's behavior in situations with unfamiliar people. Parents are provided with these descriptions and instructed to choose the description that applies best to their child (e.g., high behavioral inhibition = "As long as I remember, my child is shy when he/she has to talk to an unfamiliar person. On such occasions, he/she is nervous, he/she is not able to laugh, and he/she does not know 
what to say"). Note that the BIQ only contains items referring to social behavioral inhibition. In the current study, the children were qualified as inhibited when their BIS total score was higher than 22 or when their parents chose the description of high behavioral inhibition on the BI categories. Control children's BIS scores were 12, 13, or 14 and these children were never identified as high on behavioral inhibition on the $\mathrm{BI}$ categories. Previous research has yielded support for the reliability and validity of the BIS. To begin with, in a recent study by Van Brakel et al. (2004), moderate but significant relations were found between parent reports of behavioral inhibition as measured by the BIS and an observational index of this temperamental trait, thus providing evidence for the validity of the scale. Further, an investigation by Muris et al. (2003a) has shown that parent ratings on the BIS had acceptable correlations (with $r$ 's between .44 and .49) with children's self-reported BIS scores. Finally, the reliability of the BIS appears good: Cronbach's alphas are well above .80 , and the test-retest correlation is .77 when measured over a 2-year period (see Van Brakel \& Muris, 2006) ${ }^{1}$.

The Attachment Style Questionnaire (ASQ; Hazan \& Shaver, 1987) consists of three descriptions concerning children's feelings about and perceptions of their relationships with other children. Parents are provided with these descriptions and instructed to choose the description that applies best to their child. In this way, they classify their children as either securely, avoidantly, or ambivalently attached. In the present study, the ASQ scores were dichotomized: that is, secure attachment was recoded as 0 , whereas avoidant and ambivalent attachment were both recoded as 1 (insecure attachment). In a study by Muris et al. (2001), the connection between the child version of the ASQ and a concurrent measure of attachment, the Inventory of Parent and Peer Attachment (IPPA; Armsden \& Greenberg, 1987), was examined. Results showed that adolescents who classified themselves as securely attached on the ASQ displayed a higher quality of attachment to both parents and peers than adolescents who classified themselves as insecurely (i.e., avoidantly or ambivalently) attached on the AQ. Clearly, this finding supports the validity of the ASQ (see also Muris \& Meesters, 2002).

\footnotetext{
'In the present study, the BIS correlated significantly with observational measures of the inhibited temperament on occasion I, with r's ranging between 0.21 and 0.26 (see also Van Brakel et al., 2004). Correlations between the BIS on occasion II and the observational measures on occasion I were also significant and ranged between 0.17 and 0.20 .
} 
The Modified EMBU (Egna Minnen Beträffende Uppfostran, which is Swedish for My memories of upbringing; Castro, Toro, Van der Ende, \& Arrindell, 1993; Muris, Meesters, \& Van Brakel, 2003b) consists of 40 items that can be allocated to four types of parental rearing: emotional warmth, rejection, control, and anxious rearing. On the parent version of this scale, parents provide an indication of their own rearing behaviors. Each item is scored on a 4-point Likert scale: $1=$ No, never, 2 = Yes, but seldom, 3 = Yes, often, 4 = Yes, most of the time. The psychometrics of the modified EMBU were tested in a large sample of children and adolescents $(N=1702)$. Results showed that the scale has a clear-cut 4-factor structure, which is in correspondence with the hypothesized subscales. Furthermore, EMBU scales were reliable in terms of internal consistency and test-retest stability (Muris et al., 2003b). In the current study only two EMBU subscales were used, namely control (e.g., "When your child comes home, he/she has to tell you what he/she has been doing") and anxious rearing (e.g., "You are scared when your child does something on his/her own"), as these seem most relevant in relation to anxiety symptoms (Muris et al., 2003b).

The Screen for Child Anxiety Related Emotional Disorders (SCARED; Birmaher, Khetarpal, Brent et al., 1997; Muris et al., 1999) is a questionnaire that attempts to measure childhood anxiety symptoms in terms of the Diagnostic and Statistical Manual of Mental Disorders (DSM-IV; American Psychiatric Association, 1994). Item examples are "My child worries about things working out for him/her" (generalized anxiety disorder), "My child doesn't like being away from his/her family" (separation anxiety disorder), "When my child is frightened, his/her heart beats fast" (panic disorder). Parents have to indicate how frequently they think their child experiences each symptom on a 3 -point scale: 0 =almost never, $1=$ sometimes, and 2 = often. SCARED total and subscale scores can be obtained by summing across relevant items. In the current study, we were particularly interested in two scores. These scores indicated the distinction between social anxiety (the sum of items referring to social phobia) and anxiety problems in general (the sum of all other items). Previous research has demonstrated that the SCARED has good internal consistency (e.g., Muris, Schmidt, \& Merckelbach, 2000b; Birmaher et al., 1997), test-retest reliability, and discriminant validity (e.g., Birmaher et al., 1997). Furthermore, a recent study by Muris and colleagues (2004) has demonstrated that SCARED scores have considerable potential for predicting specific DSM-defined anxiety disorders in non-clinical and clinically referred children and adolescents (Muris, Dreessen, Bögels, Weckx, \& Van Melick, 2004; Muris, Merckelbach, Kindt et al., 2001). 
The Life Experiences Survey (LES; Sarason, Johnson \& Siegel, 1978) is originally a 57- item measure that allows respondents to indicate events that they have experienced during the past year. Subjects are asked to indicate those negative and positive events experienced during the past year as well as the perceived impact of each particular event. The current study only focused on negative life experiences. In other words, this measure asked parents which negative events had occurred in the life of their child during the past year, and to indicate the perceived impact of that event on a 3 -point scale with $1=$ a little stress, $2=$ average stress, and $3=a$ lot of stress. A total stress score was obtained by summing stress levels across experienced items. The original version of the LES has satisfactory psychometric properties (Sarason et al., 1978).

The Dominic-R (Valla, Bergeron, Bidaut-Russell, St-Georges, \& Gaudet, 1997) is a pictorial, fully structured, DSM-III-R-based questionnaire to assess symptoms of the 7 prevalent DSM-III-R disorders in children: attention-deficit hyperactivity disorder (ADHD), oppositional defiant disorder (ODD), conduct disorder $(C D)$, major depressive disorder (MDD), separation anxiety disorder (SAD), overanxious disorder (OAD), and simple phobia (SPh). In the present study, we were particularly interested in social phobia (SOC), and so we added 8 items to cover the symptoms of this anxiety disorder. The Dominic- $R$ can be reliably administered by lay interviewers (Valla, Bergeron, \& Smolla, 2000). Items (e.g., "Do you feel sick when your parents leave without you, just like Dominic?", "Are you afraid of meeting unfamiliar people, just like Dominic?") have to be answered on a yes/no scale (no = 0 , yes $=1$ ). For each Dominic-R subscale a score can be obtained by summing across relevant items. Again the distinction between social anxiety (the sum of items referring to social phobia) and anxiety problems in general (the sum of items referring to the other anxiety scales) had our special interest. Valla and colleagues (2000) report that the test-retest reliability of the Dominic-R and the criterion validity against clinical judgment are adequate. In the present study we employed a parent version of the Dominic- $R$, which means that all questionnaire items were rephrased in terms of the parents' perspective (e.g. "Does your child worry a lot about not having friends?").

The Dutch translation of the Spielberger State-Trait Anxiety Inventory (STAI; Spielberger, Gorsuch, \& Lushene, 1970; Van der Ploeg, Defares, \& Spielberger, 1979) was used to assess trait anxiety in the parents. This scale consists of 20 statements (e.g., "I feel tense and restless") of which subjects have to indicate the frequency on a 4 -point scale: 1 = almost never, 2 = sometimes, $3=$ often, $4=$ almost always. The reliability and validity of the Dutch translation of the STAI have 
shown to be as good as the original form (Van der Ploeg, 1980; Spielberger, Gorsuch, Lushene, Vagg, \& Jacobs, 1983 ).

\section{Statistical analysis}

The Statistical Package for Social Sciences (SPSS) was used for computing descriptive statistics, correlation coefficients and carrying out $t$-tests. Path analysis was used to examine whether (a) behavioral inhibition would be predictive of anxiety symptoms, especially social anxiety symptoms, from one to another point-in-time, (b) behaviorally inhibited children who experienced high levels of stress as a result of adverse life events would be particularly prone to display an increase of anxious symptoms, (c) insecure attachment, an anxious and overprotective parenting style, and parental anxiety would play a unique role in the development of children's anxious symptoms, and (d) these vulnerability factors interacted with behavioral inhibition.

Path analysis was carried out using LISREL package 8.54. All the variables used in the path models were first examined by means of a missing value analysis (SPSS 14.0.2). Subjects with more than 6 missing variables were excluded from further analysis. The missing values from the subjects with 1 to 6 missing variables were predicted using the linear regression analysis with residuals estimation adjustment of SPSS. Fifty-six subjects were excluded on the basis of the abovementioned criterion, which means that 206 subjects (88 behavioral inhibited children and 118 controls) were used in the final data analysis. The following goodness-of-fit statistics were used: the Standardized Root Mean Squared Residual (SRMR) which should be 0.08 or smaller, and the Comparative Fit Index (CFI) which should be larger than .90 in order to have a relatively good fit between the hypothesized model and the observed data (Hu \& Bentler, 1999).

The strategy used for the structural equation modeling analyses of the comprehensive longitudinal model was as follows. First, all variables measured across relevant occasions were entered in the model in order to study the unique main effects of various risk factors on symptom scores. Secondly, all theoretically meaningful interaction effects were added to the model. It is important to note that a parsimonious model was construed that retained the significant main and interaction effects of various risk factors on the main outcome variables under study. Thus, the final model only included the significant paths that were obtained with the path analyses described above. The covariances between the dependent variables were set free during the analysis. 


\section{RESULTS}

\section{General findings}

Before addressing the main issues of the current study, a number of general findings are briefly discussed. First, as can be seen in Table 2, all questionnaires were reliable in terms of internal consistency, with Cronbach's alphas ranging between 0.82 and 0.96. Second, $t$-tests revealed that there was one significant gender difference: on occasion II, boys displayed higher social phobia scores than girls, means being 1.7 $(S D=2.05)$ vs. $1.2(S D=1.59)[t(217)=2.39, p<.001]$.

Table 2: Mean scores (standard deviations) and Cronbach's alphas for all questionnaires that were used in the current study

\begin{tabular}{|c|c|c|}
\hline Questionnaires & $M(S D)$ & Cronbach's $\alpha$ \\
\hline \multicolumn{3}{|l|}{ Screening } \\
\hline$B I Q$ & 18.9 (6.6) & 0.91 \\
\hline \multicolumn{3}{|l|}{ Occasion I } \\
\hline $\begin{array}{l}\text { BIQ } \\
\text { DOMINIC } \\
\text { EMBU } \\
\text { STAI mother } \\
\text { STAI father }\end{array}$ & $\begin{array}{l}17.7(6.3) \\
14.8(9.8) \\
43.9(6.2) \\
35.1(8.9) \\
32.2(9.0)\end{array}$ & $\begin{array}{l}0.96 \\
0.89 \\
0.82 \\
0.91 \\
0.93\end{array}$ \\
\hline \multicolumn{3}{|l|}{ Occasion II } \\
\hline $\begin{array}{l}\text { BIQ } \\
\text { DOMINIC } \\
\text { EMBU } \\
\text { STAl mother } \\
\text { STAl father } \\
\text { ASQ insecure } \\
\text { LES }\end{array}$ & $\begin{array}{l}16.9(6.3) \\
13.8(10.3) \\
42.7(6.5) \\
33.6(8.9) \\
31.8(8.1) \\
37(15.9)^{a} \\
2.6(3.0)\end{array}$ & $\begin{array}{l}0.96 \\
0.89 \\
0.83 \\
0.93 \\
0.92 \\
b \\
b\end{array}$ \\
\hline \multicolumn{3}{|l|}{ Occasion III } \\
\hline SCARED & $26.6(19.0)$ & 0.95 \\
\hline \multicolumn{3}{|c|}{$\begin{array}{l}\text { Note. } N=262 ; B I Q=\text { Behavioral Inhibition Questionnaire; EMBU = Egna Minnen Beträffende } \\
\text { Uppfostran, which is Swedish for My memories of upbringing; STAI = trait version of the State- } \\
\text { Trait Anxiety Inventory; ASQ = Attachment Style Questionnaire; SCARED = Screen for Child } \\
\text { Anxiety Related Emotional Disorders; LES = Life Experiences Survey; DOMINIC = questionnaire } \\
\text { to assess symptoms of the } 7 \text { prevalent DSM-III-R disorders in children. a Attachment was } \\
\text { measured by means of a } 1 \text {-item measure: The number (percentage) of children classified as } \\
\text { insecurely attached is shown. }{ }^{b} \text { Cronbach's alpha could not be computed for these measures. }\end{array}$} \\
\hline
\end{tabular}




\section{Correlational analyses}

Table 3 presents the relationships among behavioral inhibition, parental rearing, and parental anxiety on occasion I, and behavioral inhibition, social anxiety, other anxiety disorders, depression, and externalizing problems on occasion II. Only the most important relationships will be discussed here. First of all, results demonstrate that the BIS scores were stable over a 1 -year period, with a test-retest correlation of 87 . Second, the correlation between behavioral inhibition and social anxiety was as anticipated $(r=.71)$. Behavioral inhibition was also associated with other anxiety disorder symptoms $(r=.28)$, although it should be mentioned that the correlation between behavioral inhibition and social anxiety was significantly larger than the correlation between behavioral inhibition and other anxiety disorder symptoms ( $Z=$ 7.70, $p<.001)$. Behavioral inhibition was significantly related to symptoms of depression $(r=.21)$, but did not show a significant association with externalizing problems $(r=-.02)$. Third, the results indicate that the other risk factors that were assessed on occasion I were also related to anxiety and other psychopathological symptoms as measured on occasion II. More specifically, anxious rearing not only showed a significant correlation with other anxiety disorders $(r=.39)$, but also with

Table 3: Correlations between behavioral inhibition, parental rearing, and parental anxiety on occasion I, and behavioral inhibition, social anxiety, other anxiety disorders, depression, and externalizing problems on occasion II

Occasion II

\begin{tabular}{lllll} 
BIQ & DOMINIC & & \\
\hline $\begin{array}{l}\text { Behavioral } \\
\text { inhibition }\end{array}$ & $\begin{array}{l}\text { Social } \\
\text { anxiety }\end{array}$ & $\begin{array}{l}\text { Other } \\
\text { anxiety } \\
\text { disorders }\end{array}$ & Depression & $\begin{array}{l}\text { Externalizing } \\
\text { problems }\end{array}$ \\
\hline
\end{tabular}

Occasion 1

$B I Q$

$.87^{* *}$

$.71^{* *}$

$.28^{* *}$

$.27^{*}$

$-.02$

EMBU

Anxious rearing

.07

.00

.10

$-.03$

$.39 * *$

.07
$.37^{* *}$

.11
$.47^{* *}$ .13*

STAI

Note. $N=262 ; B I Q=$ Behavioral Inhibition Questionnaire; EMBU = Egna Minnen Beträffende Uppfostran, which is Swedish for My memories of upbringing; STAI = trait version of the StateTrait Anxiety Inventory; DOMINIC = questionnaire to assess symptoms of the 7 prevalent DSMIII-R disorders in children. ${ }^{* *} p<0.01,{ }^{*} p<0.05$. 
depression and externalizing problems ( $r$ 's being .37 and .41 , respectively). Further, in particular trait anxiety of the mother was related to behavioral inhibition and all psychopathology measures on occasion II ( $r$ 's ranging between .21 and .39).

Table 4 displays the relationships between behavioral inhibition, parental rearing, attachment, parental anxiety, and life events on occasion II and social anxiety and other anxiety disorder symptoms, as measured by the SCARED, on occasion III. Note that, behavioral inhibition on occasion II was related to both social anxiety and other anxiety disorder symptoms on occasion III ( $r^{\prime}$ s being .72 and .34 respectively), with the former being again stronger than the latter correlation ( $Z=$ 8.84, $p<.001$ ). In addition, other risk factors measured on occasion II (anxious rearing, attachment, parental anxiety, and life experiences) also showed significant correlations with social anxiety as well as with symptoms of other anxiety disorders on occasion III ( $r$ 's ranging between .13 and .35).

Table 4: Correlations between behavioral inhibition, parental rearing, attachment, parental anxiety, and life events on occasion II, and social anxiety and other anxiety disorder symptoms on occasion III

\begin{tabular}{|c|c|c|}
\hline & Occasion III & \\
\hline & SCARED & SCARED \\
\hline & Social Anxiety & Other Anxiety Disorders \\
\hline Occasion II & & \\
\hline$B I Q$ & $.72 * *$ & $.34^{* *}$ \\
\hline EMBU & & \\
\hline Anxious Rearing & $.13^{*}$ & $.23^{* *}$ \\
\hline Overprotection & -.10 & -.04 \\
\hline$A S Q$ & & \\
\hline Attachment & $.35^{* *}$ & $.28^{* *}$ \\
\hline STAl & & \\
\hline Mother & $.33^{* *}$ & $.33^{* *}$ \\
\hline Father & $.73^{*}$ & $.20^{* *}$ \\
\hline LES & & \\
\hline Life experiences & $.19 * *$ & $.33^{* *}$ \\
\hline $\begin{array}{l}\text { Note. } N=262 ; B I Q \\
\text { Uppfostran, which is } \\
\text { Trait Anxiety Inventor } \\
\text { SCARED = Screen fo } \\
{ }^{* *} p<0.01,{ }^{*} p<0.0\end{array}$ & $\begin{array}{l}\text { oral Inhibition } \\
\text { or My memor } \\
\text { Attachment } S \\
\text { kiety Related E }\end{array}$ & $\begin{array}{l}\text { ABU = Egna Minnen Beträffende } \\
S T A I=\text { trait version of the State- } \\
; \angle E S=\text { Life Experiences Survey, }\end{array}$ \\
\hline
\end{tabular}

The correlations between behavioral inhibition, parental rearing, and parental anxiety on occasion I, and symptoms of social anxiety and other anxiety disorders as measured by the SCARED on occasion III are shown in Table 5. 
Table 5: Correlations between behavioral inhibition, parental rearing and parental anxiety on occasion I and social anxiety and other anxiety disorder symptoms on occasion III

\begin{tabular}{|c|c|c|}
\hline & \multicolumn{2}{|l|}{ Occasion III } \\
\hline & SCARED & SCARED \\
\hline & Social Anxiety & Other Anxiety Disorders \\
\hline \multicolumn{3}{|l|}{ Occasion I } \\
\hline$B I Q$ & $.66^{* *}$ & $.29 * *$ \\
\hline \multicolumn{3}{|l|}{$E M B U$} \\
\hline Anxious Rearing & .71 & $.26^{* *}$ \\
\hline Overprotection & -.04 & .05 \\
\hline \multicolumn{3}{|l|}{ STAI } \\
\hline Mother & $.32 * *$ & $.32 * *$ \\
\hline Father & .03 & .08 \\
\hline \multicolumn{3}{|c|}{$\begin{array}{l}\text { Jote. } N=262 ; B I Q=\text { Behavioral Inhibition Questionnaire; EMBU = Egna Minnen Beträffende } \\
\text { Ippfostran, which is Swedish for My memories of upbringing; STAI = trait version of the State- } \\
\text { rait Anxiety Inventory; SCARED = Screen for Child Anxiety Related Emotional Disorders; }{ }^{* *} p< \\
.01,{ }^{*} p<0.05 \text {. }\end{array}$} \\
\hline
\end{tabular}

The results show that behavioral inhibition on occasion I was strongly linked to social phobia and, to a lesser extent, to other anxiety disorders some 3 years later ( $r$ 's being .66 and .29 respectively; $Z=8.13, p<.001)$. Second, some of the other risk factors also had long-term relationships with anxiety symptoms. More precisely, anxious rearing was significantly related to other anxiety disorders $(r=.26)$, whereas trait anxiety of the mother was connected to symptoms of social phobia $(r=.32)$ as well as other anxiety disorders $(r=.32)$.

\section{Structural Equation Modeling}

Figure 1 shows the path model that examined the specificity of behavioral inhibition in predicting symptoms of anxiety disorders and in particular social anxiety as well as other psychopathological symptoms (i.e., from occasion I to II). As can be seen in this figure, behavioral inhibition predicted social anxiety, but failed to predict other anxiety disorder symptoms, depression, or externalizing problems. The fit indices for this model were reasonable (CFI $=0.89$; $S R M R=0.10)$, and so it can be concluded that behavioral inhibition only acted as a specific risk factor for the development of social anxiety. 


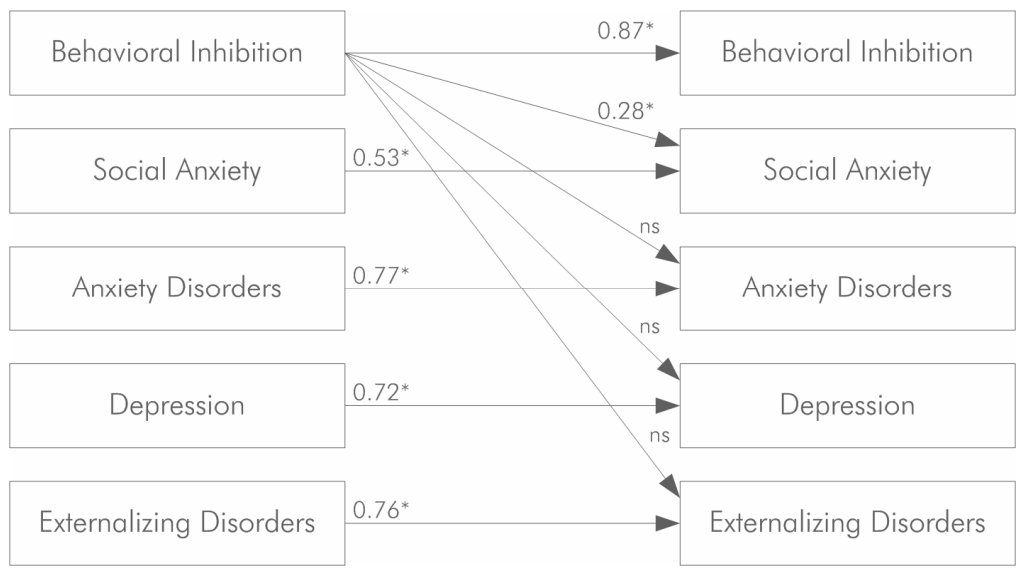

Figure 1. Results of the path analysis examining the specificity of behavioral inhibition in predicting symptoms of anxiety disorders and in particular social anxiety as well as other psychopathological symptoms from occasion I to II

Note. Path coefficients represent standardized beta estimates. ${ }^{*} p<.05$ level. Comparative Fit Index $(C F I)=0.89$ and $S R M R=0.10$.

Figure 2 shows the path analysis examining the influence of behavioral inhibition in interaction with life events on the development of social anxiety, other anxiety disorder symptoms, and depression ${ }^{2}$ in children during a 1 -year period (i.e., from occasion I to occassion II). The final model was found to have an acceptable fit $(C F I=0.90 ; S R M R=0.07)$. Note that no moderating effects of 'life events' on the link between behavioral inhibition and any of the anxiety/psychopathology scores were found. That is, 'life events' did not interact with behavioral inhibition, but rather acted as an independent factor influencing the development of other anxiety disorders and depression.

2 Depression was included as an outcome variable in this analysis in order to replicate the findings of the Brozina and Abela (2006) study. The purpose of that study was (1) to examine the relationship between behavioral inhibition and anxious symptoms within a diathesis-stress framework and (2) to examine whether behavioral inhibition acted as a specific vulnerability to anxious symptoms, or as a common vulnerability factor to both anxious and depressive symptoms. 


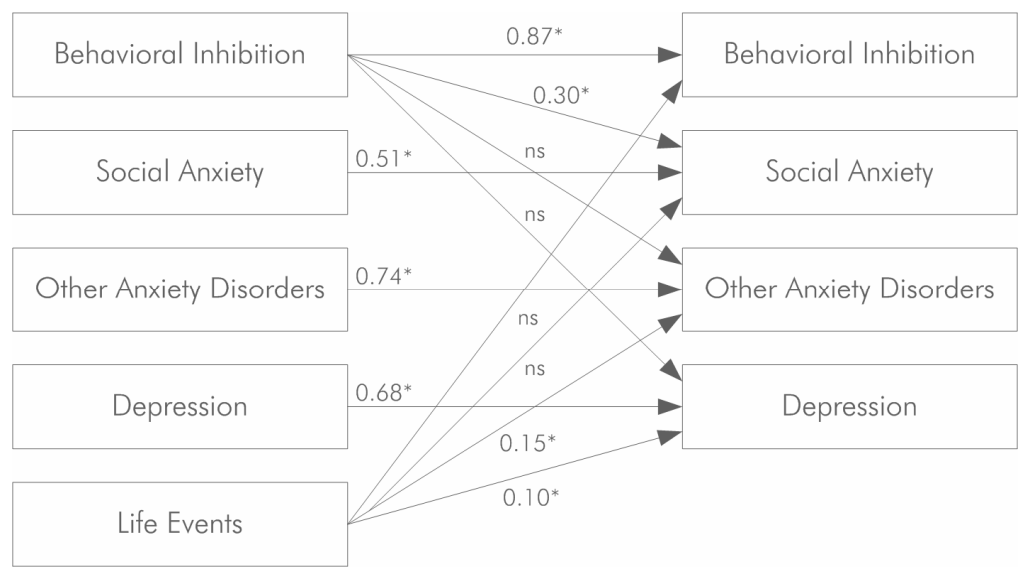

Figure 2. Results of the path analysis examining the influence of behavioral inhibition, life events, and their interaction on the development of social anxiety, other anxiety disorder symptoms, and depression in children during a 7 -year period (i.e., from occasion I to II)

Note. No moderating effect of 'life events' on the relation between behavioral inhibition and anxiety and depression symptoms was found. Path coefficients represent standardized beta estimates. ${ }^{*} p<.05$ level. Comparative Fit Index $(C F I)=0.90$ and SRMR $=0.07$.

Figure 3 (the final model only included the significant paths that were obtained with the path analyses described above) shows the best fitting model (CFI = 0.89 ; SRMR $=0.06$ ) of the direct and moderating paths reflecting the influence of various risk factors on children's level of social anxiety and other anxiety disorder symptoms during the entire 3-year follow-up period. Note that across all occasions behavioral inhibition, social anxiety, and other anxiety disorder symptoms remained fairly stable (all beta's between 0.31 and 0.88 ). Most importantly, it was found that behavioral inhibition on occasion I had a unique effect in predicting social phobia on occasion II (beta $=0.28$ ), whereas behavioral inhibition on occasion II had a independent influence on social phobia and other anxiety disorders on occasion III (beta $=$ respectively 0.45 and 0.12 ). Of course, these findings underline the role of behavioral inhibition in the development of childhood anxiety symptoms and in particular symptoms of social phobia. 


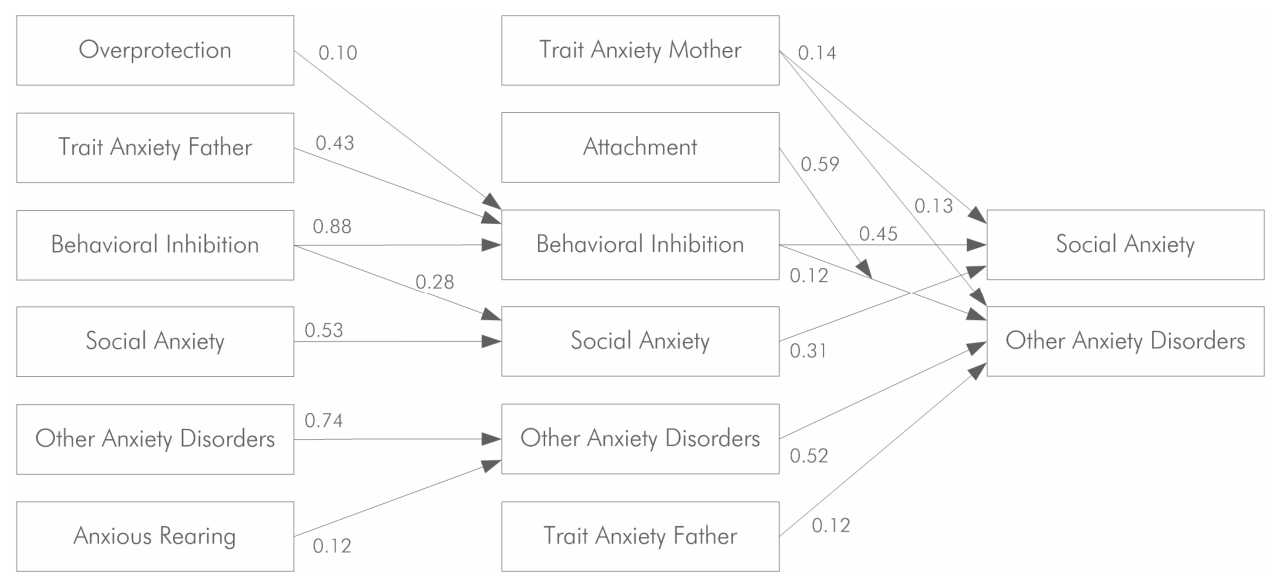

Figure 3. Results of the path analysis testing direct and moderating paths of various risk factors on the development of social anxiety and other anxiety disorder symptoms in children during the 3-year follow-up period

Note. Path coefficients represent standardized beta estimates. All of the path coefficients are significant beyond the $p<.05$ level. Comparative Fit Index (CFI) $=0.89$ and SRMR $=0.06$.

Note further that a number of risk factors made significant additional contributions. For example, parental rearing style predicted behavioral inhibition and some of the psychopathological symptoms in this model. That is, overprotection on occasion I had a small but unique effect on behavioral inhibition on occasion II (beta $=.10$ ), while anxious rearing on occasion I significantly predicted symptoms of other anxiety disorders on occasion II (beta $=0.12$ ). In addition, trait anxiety of mother and father as measured on occasion II, each uniquely predicted anxiety symptoms on occasion III. More precisely, a significant path emerged between trait anxiety of the father on occasion II and other anxiety disorders on occasion III (beta $=0.12$ ) as well as between trait anxiety of the mother on occasion II and social phobia/other anxiety disorders on the last occasion (beta's being 0.14 and 0.13 ).

Only two significant interaction effects were found. To begin with, behavioral inhibition and trait anxiety of the father on occasion I had an interactive effect on behavioral inhibition on occasion II (beta $=.43$ ), which indicated that high behavioral inhibited children with an anxious father displayed the highest behavioral inhibition levels on occasion II. Second, behavioral inhibition and attachment on occasion II had an interactive effect on other anxiety disorders on occasion III (beta 
$=.59)$. That is, children who were defined as high on behavioral inhibition and insecurely attached displayed the highest levels of anxiety symptoms over time, whereas children who were classified as low on inhibition and securely attached on occasion II exhibited the lowest anxiety levels.

\section{DISCUSSION}

The present study examined the additive and interactive effects of behavioral inhibition and other risk factors on the development of childhood anxiety symptoms using a longitudinal research design. Moreover, it was investigated whether behavioral inhibition plays a role in the development of social anxiety, anxiety problems in general, or a broad range of psychopathological symptoms. The main results of the study can be summarized as follows. Consistent with the hypothesis that behavioral inhibition is a rather specific vulnerability factor, the results indicated that high levels of this temperamental factor were particularly associated with an increase of social anxiety symptoms over time. The longitudinal associations between behavioral inhibition and other anxiety problems were clearly less strong, whereas prospective connections with other psychopathological symptoms were not found. Thus, the current findings seem to justify the conclusion that behavioral inhibition primarily acts as a specific risk factor for the development of social anxiety symptoms.

Second, the current study demonstrated that besides behavioral inhibition a number of other risk factors made significant additional contributions in the model explaining anxiety symptoms across the three-year period. For example, anxious rearing on occasion I significantly predicted symptoms of other anxiety disorders on occasion II (see for comparable results Grüner et al., 1999; Muris et al., 2000a; Muris, Meesters, \& Van Brakel, 2003b), whereas trait anxiety of both parents as measured on occasion II made independent contributions to anxiety symptoms on occasion III. The latter finding is in line with a study of Biederman and colleagues (1991) who showed that children of high anxious parents run a greater risk for developing anxiety disorders themselves.

Further, some evidence emerged showing that risk factors interacted with each other, thereby creating an increased vulnerability for developing anxiety. That is, behavioral inhibition and attachment on occasion II had an interactive effect on other anxiety disorders on occasion III. That is, children who were defined as high on behavioral inhibition and insecurely attached displayed the highest levels of anxiety symptoms over time, whereas children who were classified as low on inhibition and securely attached on occasion II exhibited the lowest anxiety levels. This result adds 
to earlier studies showing that children who combine a behaviorally inhibited temperament with an insecure attachment status tend to display the highest levels of anxiety disorders symptoms (e.g., Calkins \& Fox, 1992; Mannasis et al., 1995; Muris et al., 2000a; Muris \& Meesters, 2002, Shamir-Essakow et al., 2005), which shows that individual risk factors may influence each other, thereby yielding an even greater risk for the development of anxiety pathology in youths.

Moreover, the final model showed that not only anxiety symptoms were predicted by various risk factors, behavioral inhibition itself was also influenced by some of the vulnerability factors. That is, overprotection on occasion I had a small but unique effect on behavioral inhibition on occasion II. Further, behavioral inhibition and trait anxiety of the father on occasion I had an interactive effect on behavioral inhibition on occasion II, indicating that high behaviorally inhibited children with an anxious father displayed an increase of behavioral inhibition levels on occasion II. These findings are in keeping with the notion that overprotective/anxious parents may hinder their behaviorally inhibited children from developing effective coping strategies and acquiring adequate social skills (Rapee, 1997), which is likely to result in avoidance of 'anxious' situations. As a consequence, these children remain inhibited or even become more inhibited (Rubin et al., 1997, 1999; Van Brakel et al., 2006). Note that the current data suggest that father might play a specific role in this respect: if he shows high levels of anxiety, the inhibited child is particularly prone to continue or even intensify his/her temperamental vulnerability.

Finally, the current study tested the idea (consistent with a diathesis-stress framework) that behaviorally inhibited children who experienced high levels of stress as a result of adverse life events would be particularly prone to display an increase of anxious symptoms. This hypothesis could not be confirmed. The results showed that 'life events' did not interact with behavioral inhibition in the prediction of symptom levels, but rather acted as an independent factor influencing the development of depression and other anxiety disorders. In contrast, Brozina and Abela (2006) found that stress (as a result of adverse life events) moderated the relationship between behavioral inhibition and anxiety symptoms. That is, children with high behavioral inhibition showed increases in anxious symptoms when they experienced high levels of stress. A possible explanation for these contrasting findings could be, first of all, the length of the follow-up period (i.e., 6 weeks in the Brozina and Abela study versus 1 year in the current study). It might be possible that a period of 1 year is too long to retrospectively assess stress levels in a reliable and valid way. Moreover, temporary effects may have occurred within the 1 -year period, but may not have 
been measured because the assessment of life events was too global. Second, it may well be that the children in the present study were too young to really experience the negative impact of these adverse life events. Third and finally, the measures that were used in both studies differed. That is, whereas the current investigation relied on an index listing serious but rather infrequent life events (e.g., death of a parent, financial problems, divorce of parents), Brozina and Abela employed a scale measuring daily hassles, which simply may have been more sensitive to assess children's levels of experienced stress.

Altogether, the recent findings confirm the validity of etiological models which assume that the pathogenesis of anxiety can best be conceptualized as a dynamic interplay of various predisposing factors. Vasey and Dadds (2001) have described such a multifactorial framework for the development of childhood anxiety disorders. Of particular importance in their model are the reciprocal connections between the different risk and protective factors within a developmental context.

\section{Limitations}

Several limitations of the current study should be considered when interpreting the current results. First, we did not assess diagnoses of anxiety disorders and other mental disorders (e.g., depression). Nevertheless, it should be mentioned that the SCARED which was employed as the primary outcome measure, provides a good indication of various DSM-defined anxiety disorders in non-clinical and clinically referred children and adolescents (Muris et al., 2001, 2004). Second, despite the fact that the BIS has been thoroughly investigated in previous studies (e.g., Van Brakel et al., 2004; Shatz, 2005) and turned out to be a valid and reliable measure, it predominantly measures social aspects of behavioral inhibition. Although the choice for the BIS can be defended by pointing at past research indicating that the non-social aspects of behavioral inhibition are more susceptible to developmental changes than the social aspects of behavioral inhibition (Hirshfeld-Becker et al., 2004; Van Brakel et al., 2004; Van Brakel \& Muris, 2006), the study would have gained in strength if we had included a measure that also covers the non-social aspects of the inhibited temperament (such as the Behavioral Inhibition Questionnaire; Bishop, Spence, \& McDonald, 2003), especially since there are some indications that non-social behavioral inhibition may be a more reliable predictor of 'other anxiety disorder symptoms' (e.g., Van Brakel et al., 2004). Third, most of the data on behavioral inhibition, attachment, parental rearing and psychopathological symptoms were retrieved from a single-informant (i.e., parents). As such, it is possible that the observed associations might have been elevated due to shared method 
variance. Preferably, researchers should rely on multiple informants when investigating such relationships. Note that the children in the current study were too young to reliably fill out these complex questionnaire. Fourth, despite the longitudinal set-up of the study, children were only followed for 3 years. Thus, it remains unclear how behavioral inhibition assessed at a young age relates to social phobia, various types of anxiety disorders, and depression over longer time periods. In this context, Brozina and Abela (2006) have pointed out that it is possible that the association between behavioral inhibition and depressive symptoms is not evident until adolescence when depressive disorders become more prevalent. In other words, it might be possible that our sample of children was too young to detect the development of certain types of problems. Fifth, according to current temperament researchers (see Muris and Ollendick, 2005), vulnerability to psychopathology is not only characterized by reactive temperament features such as behavioral inhibition but also by lack of regulative traits such as effortful control. That is, high levels of behavioral inhibition make children prone to develop psychological disorders, but it may well be the case that the negative impact of this temperament variable can be buffered by efforfful control. The present study mainly focused on reactive temperament (behavioral inhibition) but it may well be that 'effortful control' accounts for some of the variation in the development of anxiety symptoms within our group of behavioral inhibited children. Sixth and finally, another issue which needs attention is the possible tautological nature of the link between (social) behavioral inhibition and social anxiety. Note, however, that the current data demonstrate that even when controlling for social anxiety on previous assessment occasions, behavioral inhibition still remained a significant predictor of subsequent social anxiety symptoms.

\section{Conclusions}

Despite these limitations, the current data provide support for the notion that various predisposing factors have additive and, to some extent, interactive effects on the pathogenesis of anxiety in children. Further, the present findings suggest that behavioral inhibition should be primarily viewed as a specific risk factor for the development of social anxiety in children rather than a general vulnerability factor for a broad range of anxiety problems, although there might be clear methodological issues (i.e., age of the children, use of the BIS for measuring behavioral inhibition) that may have biased our results. Future research might address the role of reactive and regulative factors when examining the predictive value of temperament on the development of anxiety and other psychopathological symptoms in children (e.g., 
Calkins \& Fox, 2002; Lonigan \& Phillips, 2001; Muris \& Ollendick, 2005). In addition, more prospective studies are needed in which children are followed for longer periods of time, preferably from birth to adulthood. In this way, individual developmental trajectories could be elucidated, and clinical, cognitive, and developmental information might be further integrated in a comprehensive model on the development of childhood anxiety disorders. 

CHAPTER 7

Summary and Conclusions 


\section{SUMMARY AND CONCLUSIONS}

\section{Aims of this dissertation}

This thesis was designed (1) to study the role of behavioral inhibition in the development of anxiety disorders in children, (2) to examine the specificity of behavioral inhibition in predicting social anxiety as opposed to anxiety disorder symptomatology in general or even other types of psychopathology (e.g., depression), and (3) to investigate how behavioral inhibition interacts with other risk factors (i.e., insecure attachment, parental anxiety, negative rearing behaviors, and life events) in the pathogenesis of childhood anxiety problems.

These questions were investigated in an experimental study (Chapter 4), in a cross-sectional study (Chapter 5), and in a longitudinal study (Chapter 6). Furthermore, Chapter 2 and 3 describe two psychometric studies in which the validity and reliability of the Behavioral Inhibition Scale were investigated. The studies are summarized and briefly discussed below. The results are evaluated in the light of other relevant findings from child psychopathology research and adjacent fields. Some concluding remarks and suggestions for future research will be given.

In Figure 1 (dotted arrows), the model is shown which was already presented in the general introduction of this thesis and which has been the point of departure for the research described. The starting point in this model is a genetic biological vulnerability to sympathetic arousal. This innate physiological characteristic may be expressed at the behavioral level in the form of behavioral inhibition. Behaviorally inhibited children will avoid new, unfamiliar situations. Because they avoid these situations, they have fewer opportunities to acquire adequate social skills and coping strategies. Research has indicated that the child's behaviorally inhibited temperament and the rearing style of the parents influence each other (e.g., Rubin, Nelson, Hastings, \& Asendorpf, 1999; Rubin, Hastings, Stewart, Henderson, \& Chen, 1997). That is, shy, withdrawn children are often raised by parents who find it very difficult to encourage their children to explore new situations. Consequently, the avoidant behavior of the child is maintained or even becomes worse. As a result, the child remains extremely wary, develops no self-confidence or independence, and also thinks that it is normal to be anxious and withdrawn in new situations. The quality of the mother-child attachment also seems to influence the child's inhibited temperament and vice versa (e.g., Calkins \& Fox 1992). Furthermore, specific learning experiences and environmental stressors may also interact with a vulnerable, inhibited child's proneness to develop anxiety problems. The model includes parenting style and quality of attachment as risk 
factors that may contribute to the development of an anxiety disorder. However, it should be noted that these factors not only act as risk factors, but also may function as protective factors in behaviorally inhibited children. In other words, the risk that inhibited children will develop anxiety disorders diminishes when their parents, for example, encourage them to explore new situations. In general, the dotted arrows in the model indicate the hypotheses that are investigated in this thesis.

\section{Reliability and validity of the Behavioral Inhibition Scale}

Ten to $15 \%$ of the children are born with the biological predisposition of behavioral inhibition. As these children seem to be at risk for anxiety disorders, it may be relevant to develop instruments in order to detect behaviorally inhibited children before they become highly anxious. Early detection of these vulnerable children would make it possible to implement prevention programs. Such programs should focus on encouraging children to use problem-focused instead of avoidant and emotion-focused coping strategies as well as teaching parents to reduce anxious and overprotective rearing behaviors (Spence, 2001). So, given the importance of early detection of high-risk children, an economic and easy-to-administer scale for assessing behavioral inhibition in children would be very welcome.

In Chapter 2 and 3, the psychometrics of a brief questionnaire that was specifically developed for assessing behavioral inhibition in children was investigated. The main purpose of the study described in Chapter 2 was to examine the relationship between a parent and teacher version of the BIS and behavioral observations of the temperamental trait of behavioral inhibition. Children $(N=59)$, aged 6 to 10 years, participated in a series of experimental tasks assessing behavioral features of the inhibited temperament. The behavioral tasks used in this study were largely based on the procedures used by Kagan et al. (1988) who also exposed children in this age range to unfamiliar peers, adults, novel tasks, and toys in order to observe behavioral inhibition characteristics such as latency to approach, the amount of encouragement needed to perform a novel task, and lack of spontaneous talk. Results showed that the BIS (in particular the parent version) was significantly related to observational indices of behavioral inhibition. An additional aim of the study was to examine the relationship between behavioral inhibition as indexed by the BIS and observations, on the one hand, and measures of anxiety symptoms, fear, and behavioral symptoms, on the other hand. As expected, significant correlations between behavioral inhibition indices and symptoms of anxiety and withdrawal were observed, which of course provides further support for the validity of the scale. 
The study described in Chapter 3 examined the test-retest stability of the BIS in a sample of 7-to 12-year-olds consisting of inhibited and non-inhibited children $(N=83)$. Results demonstrated that the BIS scores were fairly stable over a 2-year period, with a test-retest correlation of .77. Further, BIS scores of inhibited children significantly increased over the 2-year period, whereas those of the non-inhibited comparison group significantly decreased. Finally, the BIS was in a theoretically meaningful way associated with temperamental traits as measured by the Rothbart scales. That is, results indicated that behavioral inhibition was positively linked to features of negative affectivity but negatively to temperamental traits related to extraversion and regulation. Thus, these results provide further support for the reliability and validity of the BIS. Taken together, the two studies revealed that the BIS is a brief and easy-to-administer instrument, which seems to provide a meaningful first impression of (young) children's level of behavioral inhibition.

As pointed out by Muris and Dietvorst (2006), the BIS has been recently developed and thoroughly examined by our research team (see also Muris et al., 1999, 2001, 2003), but still needs independent validation by other research groups. One other study that employed the BIS (Shatz, 2005) documented adequate psychometric properties for this instrument in a college-aged sample. An important finding of that study was that behavioral inhibition was more convincingly related to extraversion than to neuroticism. Shatz (2005) explained this result as follows: "The BIS items appear to inquire solely about an individual's comfort level and behavior in novel social situations. The items of the BIS appear to be much closer to the extraversion/sociability construct than the behavioral inhibition construct as usually defined" (p. 338). In Chapter 2, we explored a version of the BIS including nonsocial items as well, but results showed that only social items of the BIS (i.e., items referring to encounters with unfamiliar people) were associated with the observational indices of behavioral inhibition. It seems that the non-social aspects of behavioral inhibition are more susceptible to developmental changes than the social aspects of behavioral inhibition. In other words, whereas non-social and social features both seem to be valid indicators of behavioral inhibition in younger children, only social signs remain predictive of this temperament-based trait when children become older (Chapter 2 and 3; see also Hirshfeld-Becker, Biederman, \& Rosenbaum, 2004). We should keep in mind that the current item content of the BIS could be too narrow-minded when looking at the behavioral inhibition construct. So, a careful study of the non-social elements of behavioral inhibition in children of varying ages seems certainly warranted. 


\section{A biological correlate of behavioral inhibition}

The study described in Chapter 4 examined the startle reflex as a physiological marker of behavioral inhibition. Participants $(N=83)$ were 7 - to 12-year-old children who had been previously identified as inhibited or uninhibited as part of an ongoing longitudinal study on the role of behavioral inhibition in the development of anxiety disorders. An experiment was carried out to study startle modulation effects in response to novel and familiar pictures of threatening and non-threatening facial expressions in inhibited and uninhibited children. The main results can be summarized as follows. To begin with, no modulation effect was found. That is, children did not show the expected (adult-like) startle facilitation when viewing unpleasant pictures. Further, a habituation effect was found: that is, during the testing phase children responded more intensely to the first block of slides than to the second block of slides (irrespective of slide content). Finally, in contrast to our expectations, behaviorally inhibited children displayed smaller (rather than larger) eye blink magnitudes in response to novel slides as compared to uninhibited children.

The observation that till now mixed results have dominated the studies on startle responses in behaviorally inhibited children (cf. Snidman \& Kagan, 1994) led us to conclude that the startle reflex paradigm might not be the right procedure to measure the excitability of the amygdala in (behaviorally inhibited) children. Functional Magnetic Resonance Imaging (fMRI) may be a more useful and direct tool for identifying the biological underpinnings of children at risk for anxiety disorders (Vasa \& Pine, 2004).

\section{Multifactorial model}

Vasey and Dadds (2001) have described an integrative framework for conceptualizing the various pathways associated with the development of childhood anxiety disorders. The core issue in this model is the dynamic interplay of various potential predisposing factors in the pathogenesis of anxiety. Studies examining additive and interactive effects of these factors on childhood anxiety are just beginning to emerge (e.g., Calkins \& Fox, 1992; Mannasis et al., 1995; Muris et al., 2000; Muris \& Meesters, 2002). The study described in Chapter 5 was an attempt to examine the reciprocal connections among temperament, attachment, and parental rearing styles, and their unique and interactive relations to anxiety symptoms. Sixhundred-and-forty-four non-clinical children aged 11-15 years (mean age $=12.7$ years) completed questionnaires measuring behavioral inhibition, attachment, parental rearing behaviors, and DSM-defined anxiety symptoms. Results indicated that there were small to moderate positive correlations among various risk factors. 
Furthermore, modest but significant positive correlations were found between behavioral inhibition, attachment quality, and anxious and controlling rearing behaviors, on the one hand, and anxiety scores, on the other hand. That is, higher levels of behavioral inhibition, insecure attachment, and parental control and anxious rearing were associated with higher levels of anxiety symptoms. Finally, behavioral inhibition, attachment quality, parental control and anxious rearing each accounted for a small but unique proportion of the variance in anxiety disorders symptomatology. Further, a higher-order interaction effect was found between behavioral inhibition, attachment quality, and parental control. As expected, children who defined themselves as high on both behavioral inhibition and insecure attachment displayed particularly high levels of anxiety symptoms. The role of parental control, however, was less consistent. In some children (i.e., uninhibited/securely attached and inhibited/insecurely attached), high levels of this rearing factor were associated with higher anxiety levels, whereas in other children (i.e., inhibited/securely attached) high control was related to lower anxiety levels. These divergent effects of parental control on anxiety can be explained when one adopts the notion that this rearing factor has multiple faces. On the one hand control may be associated with extreme strictness, which has the negative consequence of reducing the development of autonomy. On the other hand, control has the positive consequence of structuring the child's environment. When looking at our data, it can be suggested that on the condition that inhibited children have parents who are sensitive and responsive (in such a way that caregiver and child are securely attached), a highly controlling parenting style has a positive influence on the anxiety symptoms of these children. That is, in this group of children, highly controlling parents offer just the structure these children need to help them navigate through their daily lives, and hence reduce anxiety. However, when children do not need such assistance (i.e., the uninhibited/secure group) or when children do need guidance but their parents cannot provide it (i.e., the inhibited/insecure group), control may manifest itself in its negative overprotective way and thus enhance anxiety. In a similar vein, Kochanska and colleagues (2004) recently demonstrated that early attachment security moderates the effects of maternal parenting on children's development.

\section{Behavioral inhibition as a risk factor for the development of anxiety disorders in children: A prospective study}

Chapter 6 presented a longitudinal research project examining the additive and interactive effects of behavioral inhibition and a wide range of other vulnerability 
factors in the development of pathological anxiety in youths. A group of 261 children (aged 5 to 8), 124 of who had been classified as high on behavioral inhibition and 137 control children, were followed during a 3-year period. Each year, an assessment took place to measure children's level of behavioral inhibition, anxiety disorder symptoms, other psychopathological symptoms, and a number of other vulnerability factors such as insecure attachment, negative parenting, adverse life events, and parental anxiety. In this way, it became possible to study (1) the unique and interactive effect of behavioral inhibition on the development of anxiety symptoms, and (2) whether behavioral inhibition plays a role in the development of social anxiety, anxiety problems in general, or even a broad range of psychopathological symptoms (e.g., depression).

Results obtained with Structural Equation Modeling indicated that behavioral inhibition primarily acted as a specific risk factor for the development of social anxiety symptoms, rather than a general vulnerability factor for a broad range of anxiety problems, although there might be clear methodological issues (i.e., age of the children, use of the BIS (notably its social focus) for measuring behavioral inhibition) that may have biased our results. Furthermore, the comprehensive longitudinal model showed additive as well as interactive effects for various vulnerability factors on the development of anxiety symptoms. Behavioral inhibition itself was also influenced by some of the vulnerability factors. In addition, no support was found for the hypothesis that behaviorally inhibited children who experienced adverse life events would be particularly prone to display an increase of anxious symptoms. Altogether, the recent findings confirm the validity of etiological models which assume that the pathogenesis of anxiety can best be conceptualized as a dynamic interplay of various predisposing factors. As mentioned before, Vasey and Dadds (2001) have described such a multifactorial framework for the development of childhood anxiety disorders. Of particular importance in their model are the reciprocal connections between the different risk and protective factors within a developmental context. More prospective studies are needed in which children are followed for longer periods of time, preferably from birth to adulthood. In this way, individual developmental trajectories could be elucidated, and clinical, cognitive, and developmental information might be further integrated in such a comprehensive model on the development of childhood anxiety disorders.

\section{Concluding remarks and future research}

The studies presented in this thesis demonstrate that behavioral inhibition is an important risk factor in the development of childhood anxiety disorders, and 
especially social anxiety. Parental anxiety, parenting style, attachment, and life events are shown to play either an additive or an interactive role in this process. More precisely, the black arrows in Figure 1 reflect the findings resulting from the longitudinal study (Chapter 6). A comparison of the 'dotted' and 'black models' in this figure makes clear that some of the hypothesized relationships were not addressed (e.g., specific learning experiences were not measured), or were not confirmed (e.g., no interactive effect of behavioral inhibition and environmental stressors was found). Nevertheless, note also that most of the hypothesized (prospective) relationships were confirmed in the current research. Altogether, these findings make clear that it is important to make use of multifactorial models when investigating developmental psychopathology in general, or the development of anxiety disorders in particular. Research limitations and suggestions for future research have been discussed throughout this thesis. However, there are several issues that deserve further attention.

According to current temperament researchers (see Calkins \& Fox, 2002; Muris \& Ollendick, 2005), vulnerability to psychopathology is characterized by a combination of high levels of emotionality/neuroticism and low levels of regulative temperament factors such as effortful control (i.e., regulative, executive functioningbased processes including the focusing and shifting of attention and the ability to inhibit behavior when appropriate; Rothbart, Ellis, \& Posner, 2004). That is, while it can be assumed that high levels of emotionality/neuroticism make children prone to develop psychological disorders, it may well be the case that the negative impact of this reactive temperament factor can be buffered by effortful control. Muris and Dietvorst (2006) indeed found that children high on behavioral inhibition were characterized by higher levels of neuroticism and lower levels of attention control (which is an important aspect of effortful control) as compared to children low or moderate on behavioral inhibition. Future research should focus more on 'protective factors' such as effortful control. The identification and strengthening of such factors may help children to overcome withdrawn and anxious behavior. In a similar vein, Gullone, Ollendick, and King (2006) noted that a secure attachment relationship may protect at risk children against the development of depressive symptomatology. Thus, guiding parents in reacting sensitively and adequately to their children may be a helpful strategy to prevent the occurrence of psychopathology. Another suggestion for future research made by Thompson and Raikes (2003) is the use of personoriented data-analytic strategies instead of variable-oriented approaches (see also Schott, Mason, \& Chapmen, 1999): "By contrast with variable-oriented approaches that represent individuals in terms of group means and variances, person-oriented 
approaches enable investigators to identify the proportions of individuals showing distinct risk profiles to aid in predicting the potentially diverse pathways to problematic outcomes more precisely" (p.712). A recent research report by Fox and colleagues (2005) shows us that the variability in continuity of behavioral inhibition across childhood is a function of gene-environment interactions, which are presumed to shape behavior during early development. As medical technology is moving forward, this gene-expression-methodology can be very useful in studying developmental psychopathology and at-risk behavior.

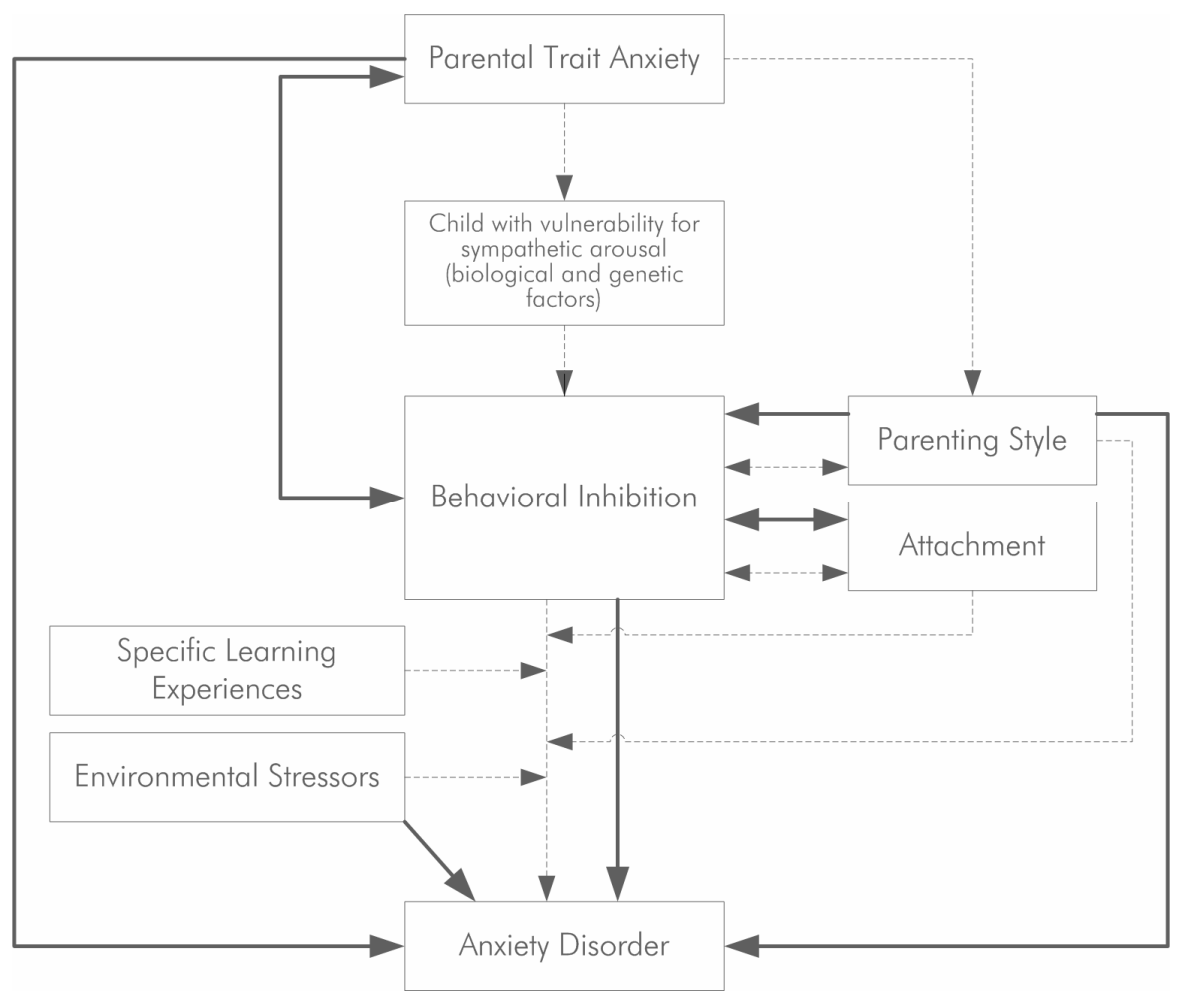

Figure 1. The role of behavioral inhibition and other risk factors in the development of anxiety disorders in children. The dotted arrows indicate the point of departure for the research described in this thesis. The black arrows reflect the results as obtained in the longitudinal study (Chapter 6) 
In conclusion, a lot of theoretically meaningful new insights have been mentioned above, which can be used in future research on the development of childhood anxiety disorders. As was stressed throughout this thesis: anxiety disorders are among the most common psychiatric diagnoses in children and adolescents (Craske, 1997), are associated with numerous negative emotions, and cause significant dysfunction in social relationships and at school (Biederman, Rosenbaum, Chaloff, \& Kagan, 1995). Further research on the dynamic interplay of various potential predisposing factors in the pathogenesis of anxiety is certainly necessary. That is, more prospective studies are needed in which children are followed from birth to adulthood, in which their genetic information is analyzed, and multiple risk as well as protective factors within the child and within the family are examined at regular points in time. 


\section{SAMENVATTING}

Dit proefschrift behandelt de volgende kwesties: (1) de rol van gedragsinhibitie in de ontwikkeling van angststoornissen bij kinderen, (2) de specificiteit van gedragsinhibitie in het voorspellen van sociale angst vergeleken met algemene angstsymptomen of zelfs andere vormen van psychopathologie (bijvoorbeeld depressie), en (3) de interactie van gedragsinhibitie met andere risicofactoren (zoals onveilige hechting, angst bij de ouder, negatieve opvoedstijlen en ingrijpende levensgebeurtenissen) in de ontwikkeling van angstproblemen bij kinderen. Deze vragen worden onderzocht in een experimentele studie (Hoofdstuk 4), in een crosssectionele studie (Hoofdstuk 5) en in een longitudinale studie (Hoofdstuk 6). Hoofdstuk 2 en 3 beschrijven twee psychometrische studies waarin de validiteit en betrouwbaarheid van de Behavioral Inhibition Scale wordt onderzocht.

Het startpunt voor bovenstaande onderzoeksvragen is een model dat schematisch is weergegeven in Hoofdstuk 1 van dit proefschrift. Het uitgangspunt in dit model is de biologische kwetsbaarheid voor sympathische arousal die genetisch overgedragen is. Deze aangeboren fysiologische kenmerken kunnen zich op gedragsniveau viten in gedragsinhibitie. Gedragsgeïnhibeerde kinderen zullen nieuwe, onvertrouwde situaties vermijden. Door het vermijden van deze situaties neemt de mogelijkheid om sociale vaardigheden en copingstrategieën te leren af. Onderzoek toont aan dat het gedragsgeïnhibeerde temperament van het kind en de opvoedingsstijl van de ouders elkaar beïnvloeden (e.g., Rubin, Nelson, Hastings, \& Asendorpf, 1999; Rubin, Hastings, Stewart, Henderson, \& Chen, 1997). Verlegen, teruggetrokken kinderen worden vaak opgevoed door ouders die het heel moeilijk vinden om hun kinderen aan te moedigen om te exploreren in nieuwe situaties waardoor het vermijdende gedrag van deze kinderen in stand blijft of zelfs erger wordt. Als gevolg hiervan maken de kinderen zich geen actieve coping-stijl eigen, blijven extreem op hun hoede, ontwikkelen geen zelfvertrouwen en onafhankelijkheid, en denken bovendien dat het heel normaal is om angstig en teruggetrokken te zijn in nieuwe situaties. Ook tussen de kwaliteit van de moederkindhechting en het geïnhibeerde temperament van het kind is er sprake van wederzijdse beïnvloeding (e.g., Calkins \& Fox 1992). Daarnaast kunnen specifieke leerervaringen van het kind en omgevingsstressoren ervoor zorgen dat het kwetsbare, geïnhibeerde kind op latere leeftijd een angststoornis ontwikkelt. In het model zijn opvoedingsstijl en de kwaliteit van de hechting opgenomen als risicofactoren die 
kunnen bijdragen aan de ontwikkeling van een angststoornis. Gebleken is dat deze factoren niet alleen een rsicofactor zijn, maar bij gedragsgeïnhibeerde kinderen ook als beschermende factor kunnen dienen. Met andere woorden, als een geïnhibeerd kind door zijn ouders aangemoedigd wordt tot exploratie, zal de kans op het ontwikkelen van een angststoornis afnemen. De piilen in het model geven de hypotheses weer die in dit proefschrift onderzocht zijn.

Tien tot $15 \%$ van de kinderen worden geboren met bovengenoemde biologische predispositie voor gedragsinhibitie (Kagan, Reznick, \& Snidman, 1988). Aangezien deze kinderen een verhoogd risico lopen op een angststoornis, lijkt het relevant om instrumenten te ontwikkelen om gedragsgeïnhibeerde kinderen te identificeren voordat ze hoog angstig zijn. Vroege detectie of identificatie van deze kwetsbare kinderen maakt het mogelijk om preventieprogramma's te implementeren. Zulke programma's zouden zich moeten richten op het aanmoedigen van kinderen om probleemgeoriënteerde copingstrategiëen te gebruiken in plaats van vermiidende- of emotiegeoriënteerde strategiëen. Bovendien zou aan ouders geleerd moeten worden om hun angstige en overbeschermende opvoedstijlen te reduceren (Spence, 2001). Gezien het belang van vroege detectie van kinderen met een verhoogd risico op het ontwikkelen van een angststoornis, is de komst van een makkelijk af te nemen instrument om gedragsinhibitie bij kinderen te meten noodzakelijk.

In Hoofdstuk 2 en 3 werden de psychometrische aspecten onderzocht van zo'n korte vragenlijst die speciaal ontwikkelt is om gedragsinhibitie bij kinderen te meten, nl. de Behavioral Inhibition Scale (BIS; Muris, Meesters, \& Spinder, 2003). De studie die beschreven wordt in Hoofdstuk 2 had als hoofddoel om de relatie te onderzoeken tussen een ouder- en leraarversie van de BIS en gedragsobservaties van de temperamentstrek gedragsinhibitie. Negeneenvijftig kinderen in de leeftiid van 6 tot 10 jaar participeerden in een aantal experimentele taakjes die gedragskenmerken van het geïnhibeerde temperament meten. De gedragstaakjes die gebruikt werden in deze studie waren grotendeels gebaseerd op de procedures die gebruikt zijn door Kagan en collega's (1988). Deze onderzoekers stelden kinderen in deze leeftijdsrange ook bloot aan onbekende leeftijdsgenootjes en onbekende volwassenen, nieuwe taakjes en nieuw speelgoed om gedragsinhibitiekenmerken te observeren zoals de tijd om een bepaald persoon of object te benaderen (latentietijd), het aantal aanmoedigingen dat nodig is om een nieuwe taak uit te gaan voeren en de afwezigheid van spontane spraak. De resultaten lieten zien dat de BIS (met name de ouderversie) significant gerelateerd is aan observatiematen van gedragsinhibitie. Deze studie onderzocht bovendien de 
relatie tussen gedragsinhibitie (bepaald door zowel de BIS als de observaties) en angstsymptomen. Zoals verwacht, werden er significante correlaties gevonden tussen gedragsinhibitie enerzijds en symptomen van angst en teruggetrokkenheid anderzijds, wat de validiteit van deze schaal ondersteunt.

De studie beschreven in Hoofdstuk 3 onderzocht de test-hertest stabiliteit van de BIS in een groep van 7- tot 12-jarigen bestaande vit geïnhibeerde en niet-geïnhibeerde kinderen $(N=83)$. De resultaten lieten zien dat de BIS scores vrij stabiel waren over een periode van 2 jaar met een test-hertest correlatie van .77. Daarbij kan opgemerkt worden dat de BIS scores van de geïnhibeerde kinderen significant toenamen tijdens de 2 jaar terwijl de scores van de niet-geïnhibeerde kinderen significant afnamen gedurende deze periode. Bovendien was de BIS in een theoretisch betekenisvolle manier geassocieerd met schalen van de Early Adolescent Temperament Questionnaire-Revised (EATQ-R; Ellis \& Rothbart, 2001). Dat wil zeggen, de resultaten gaven aan dat gedragsinhibitie positief gecorreleerd was met kenmerken van negatieve affectiviteit, maar negatief gecorreleerd met karaktertrekken gerelateerd aan extraversie en regulatie. Dus, zowel de studie in Hoofdstuk 2 als in Hoofdstuk 3 ondersteunen de betrouwbaarheid en validiteit van de BIS en laten zien dat de BIS een kort en makkelijk af te nemen vragenlijst is en een betekenisvolle eerste indruk geeft van het gedragsinhibitie niveau van een (jong) kind.

Specifieke gedragsprofielen zoals gedragsinhibitie zouden worden bepaald door overgeërfde fysiologische processen die kinderen predisponeren tot het viten van specifieke emoties en gedragingen (Kagan, Snidman, \& Arcus, 1993). Veel studies ondersteunen de hypothese dat gedragsgeïnhibeerde kinderen in aanleg een gebied in de hersenen hebben dat een zeer lage drempel heeft om geactiveerd te worden, waardoor bij onverwachte veranderingen in de omgeving of nieuwe situaties die moeilijk verwerkt kunnen worden heel snel een keten van fysiologische activiteit in werking treedt (Garcia Coll, Kagan, \& Reznick, 1984; Kagan et al., 1988; Snidman, 1989; voor overeenkomstige resultaten uit dieronderzoek zie Adamec \& StarkAdamec, 1989). Het hersengebied dat hier bedoeld wordt, beslaat de hypothalamus en het limbisch systeem, met name de amygdala. De oogknipperreflex zou individuele verschillen in amygdala excitatie kunnen meten (Schmidt \& Fox, 1998). Aangezien verondersteld wordt dat geïnhibeerde kinderen een lage drempel hebben voor amygdala activatie, onderzocht de studie welke wordt beschreven in Hoofdstuk 4 de oogknipperreflex als fysiologische marker van gedragsinhibitie. In deze studie werd een experiment uitgevoerd met 83 kinderen in de leeftijd van 7 tot 12 jaar. Dit experiment gaf de mogelijkheid de oogknipper modulatie effecten te onderzoeken 
als reactie op nieuwe (onbekende) en bekende plaatjes van dreigende en niet dreigende gezichtsuitdrukkingen bij geïnhibeerde en niet geïnhibeerde kinderen. De resultaten kunnen als volgt worden samengevat. Ten eerste werd er geen modulatie effect gevonden. Dat wil zeggen, kinderen lieten niet de verwachtte oogknipper facilitatie zien wanneer ze naar onplezierige (dreigende) plaatjes keken. Voorts vonden we een habituatie effect. Hiermee wordt bedoeld dat de kinderen tijdens de testfase intenser reageerden op de eerste plaatjes dan op de laatste plaatjes (onafhankelijk van de inhoud van de plaatjes). Ten slotte, tegen onze verwachting in, lieten geïnhibeerde kinderen kleinere oogknipperreflexen zien bii de nieuwe (onbekende) plaatjes vergeleken met niet-geïnhibeerde kinderen. We constateren dat tot op heden tegenstrijdige bevindingen het onderzoek van de oogknipperreflex bii kinderen gedomineerd hebben en dat het oogknipperreflex paradigma misschien niet de juiste methode is om de gevoeligheid van de amygdala in (gedragsgeïnhibeerde) kinderen te meten. FMRI zou een bruikbaardere methode kunnen zijn om de biologische basis te identificeren van kinderen die een verhoogd risico lopen op het ontwikkelen van een angststoornis.

In het onderzoek naar de ontwikkeling van angst bij kinderen is er vaak maar naar 1 geïsoleerde risicofactor gekeken. Tot op heden is er nog niet veel onderzoek gedaan naar de interactieve werking van meerdere factoren op de ontwikkeling van angstsymptomen. In Hoofdstuk 5 wordt een studie beschreven die de reciproke connecties onderzocht tussen temperament, hechting en opvoedstijlen en hun unieke en interactieve relatie met angstsymptomen. Zeshonderdvierenveertig niet-klinische kinderen in de leeftiid van 11 tot 15 vulden vragenlijsten in over gedragsinhibitie, hechting, opvoeding en angstsymptomen. De resultaten gaven aan dat er positieve correlaties bestonden tussen de verscheidene risico factoren. Voorts werden er positieve correlaties gevonden tussen gedragsinhibitie, kwaliteit van hechting en angstige en overbeschermende opvoedstijlen enerzijds en angstsymptomen anderzijds. Bovendien liet de data een hogere orde interactie zien tussen gedragsinhibitie, kwaliteit van hechting en een overbeschermende opvoeding. Zoals verwacht, vertoonden de kinderen die zichzelf als zowel hoog geïnhibeerd classificeerde als ook onveilig gehecht, de hoogste angstniveau's. Maar de rol van een overbeschermende opvoedstijl was minder consistent. Bij sommige kinderen (niet geïnhibeerd/veilig gehecht, geïnhibeerd/onveilig gehecht) was een overbeschermende opvoeding geassocieerd met hogere angstniveau's, terwiil bij andere kinderen (geïnhibeerd/veilig gehecht) een overbeschermende opvoeding gerelateerd was aan lagere angstniveau's. Deze divergente effecten van ouderlijke controle op angst kunnen verklaard worden door ervan vit te gaan dat deze opvoedingsfactor 
verschillende kanten heeft. Aan de ene kant kan overbescherming/controle geassocieerd worden met extreme striktheid, met de negatieve consequentie dat de ontwikkeling van de autonomie van een kind geremd wordt. Aan de andere kant, kan overbescherming/controle het positieve effect hebben van het structureren van het leven/de omgeving van het kind. Gegeven de data beschreven in de studie uit Hoofdstuk 5, kan gesuggereerd worden dat, op voorwaarde dat geïnhibeerde kinderen ouders hebben die sensitief en responsief zijn (op een dusdanige wijze dat opvoeder en kind veilig gehecht zijn), een controlerende opvoedstijl een positieve invloed heeft op de angstsymptomen van deze kinderen. Dat wil zeggen, in deze groep kinderen, overbeschermende ouders precies de structuur bieden die deze kinderen nodig hebben om hen te helpen bij het navigeren door hun dagelijkse bestaan, en als een gevolg daarvan de angst reduceert. Echter, wanneer kinderen deze assistentie niet nodig hebben (zoals de niet-geïnhibeerde/veilig gehechte groep) of wanneer kinderen deze begeleiding wel nodig hebben, maar de ouders kunnen deze niet bieden (zoals de geïnhibeerde/onveilig gehechte groep), een controlerende opvoedstijl zichzelf kan manifesteren op zijn negatieve overbeschermende manier en als gevolg daarvan angstsymptomen vergroten.

In hoofdstuk 6 wordt een longitudinaal onderzoeksproject gepresenteerd waarin de additieve en interactieve effecten van gedragsinhibitie en een aantal andere kwetsbaarheidfactoren voor de ontwikkeling van pathologische angst in kinderen onderzocht worden. Een grote groep kinderen $(N=261)$ in de leeftijd van 5 tot 8 zijn gedurende 3 jaar gevolgd. Van deze groep kinderen waren er 124 geclassificeerd als geïnhibeerd en 137 als controle kinderen. Elk jaar vond er een meting plaats om de mate te bepalen van gedragsinhibitie, angstsymptomen, andere psychopathologie symptomen, en van een aantal andere kwetsbaarheidfactoren zoals onveilige hechting, negatieve opvoedstijlen, ingrijpende levensgebeurtenissen en angst bij de ouders. Op deze manier werd het mogelijk om (1) de unieke en interactieve effecten van gedragsinhibitie op de ontwikkeling van angststoornissen bii kinderen te bestuderen, en (2) te onderzoeken of gedragsinhibitie een rol speelt in de ontwikkeling van sociale angst, van angstproblematiek in het algemeen of van een breed spectrum aan psychopathologie symptomen (zoals bijvoorbeeld depressie).

De resultaten uit de Structural Equation Modeling analyses toonden aan dat gedragsinhibitie primair acteerde als een specifieke risicofactor voor de ontwikkeling van sociale angstsymptomen in plaats van als een algemene kwetsbaarheidfactor voor een breed spectrum aan angstproblemen. Ofschoon een aantal methodologische kwesties (zoals de leeftijd van de kinderen, het gebruik van de BIS (met name zijn sociale focus) om gedragsinhibitie te meten) onze resultaten 
mogelijk hebben kunnen beïnvloeden. Voorts toonde het longitudinale model zowel additieve als interactieve effecten voor verscheidene kwetsbaarheidfactoren op de ontwikkeling van angstsymptomen. Gedragsinhibitie zelf werd ook beïnvloed door een aantal risicofactoren. Er werd geen evidentie gevonden voor de hypothese dat gedragsgeïnhibeerde kinderen die ingriipende levensgebeurtenissen hadden meegemaakt met name kwetsbaar zouden zijn voor een toename in angstsymptomen. Concluderend kunnen we stellen dat bovengenoemde resultaten de validiteit bevestigen van etiologische modellen waarin aangenomen wordt dat de pathogenese van angst het beste beschreven kan worden als een dynamisch wisselwerking van verscheidene predisponerende factoren.

Hoofdstuk 7 geeft een samenvatting van de belangrijkste bevindingen van de studies van dit proefschrift. Er worden algemene conclusies getrokken en aanbevelingen voor toekomstig onderzoek gedaan. Toekomstig onderzoek zou zich o.a. meer moeten richten op beschermende factoren. Dit zijn factoren die bijdragen om te voorkomen dat een kind met een verhoogd risico zich angstig en teruggetrokken gaat gedragen. Denk daarbii aan 'effortful control' (dat zijn executieve, regulatieve processen zoals het focussen en verschuiven van de aandacht en de mogelijkheid om bepaald gedrag zo nodig te kunnen inhiberen) en 'veilige hechting' (het sensitief en adequaat reageren van ouders op hun kind). Tot slot wordt er verwezen naar een nieuwe onderzoeksmethode die de interacties onderzoekt tussen genen en de omgeving, die verondersteld worden gedrag te vormen tijdens de vroege ontwikkeling. Geopperd wordt dat er meer prospectieve studies nodig zijn waarin kinderen vanaf hun geboorte tot aan volwassenheid gevolgd worden en waarin hun genetische informatie wordt geanalyseerd en meerdere risico- en beschermende factoren zowel binnen het kind als binnen de familie onderzocht moeten worden op meerdere momenten in de tijd. 


\section{REFERENCES}

Achenbach, T. M. (1991). Manual for the youth self-report and 1991 profiles. Burlington: University of Vermont, Department of Psychiatry.

Adamec, R.E., \& Stark-Adamec, C. (1989). Behavioral inhibition and anxiety: Dispositional, developmental, and neural aspects of the anxious personality of the domestic cat. In J.S. Reznick (Ed.), Perspectives on behavioral inhibition (pp. 93-124). Chicago: The University of Chicago Press.

American Psychiatric Association (1980). Diagnostic and Statistical Manual of Mental Disorders, $3^{\text {rd }}$ edition (DSM-III). Washington, DC: American Psychiatric Association.

American Psychiatric Association (1994). Diagnostic and statistical manual of mental disorders, fourth edition (DSM-IV). Washington, DC: American Psychiatric Association.

American Psychiatric Association (2000). Diagnositc and Statistical Manual of Mental Disorders, $4^{\text {rd }}$ edition-text revision (DSM-IV-TR). Washington, DC: American Psychiatric Association.

Andersson, K. (1999). Reactions to social and non-social novelties in 2-year-olds. Infant and Child Development, 8, 211 -228.

Armsden, G. C., \& Greenberg, M. T. (1987). The inventory of parent and peer attachment: Individual differences and their relationship to psychological well-being in adolescence. Journal of youth and adolescence, 16, 427-454.

Asendorpf, J.B. (1991). Development of inhibited children's coping with unfamiliarity. Child Development, 62, 1460-1474.

Asendorpf, J. (1990). Development of inhibition during childhood: Evidence for situational specificity and a two-factor model. Developmental Psychology, 26, 721-730.

Asendorpf, J.B. (1993). Beyond temperament: a two-factorial coping model of the development of inhibition during childhood. In K.H. Rubin \& J. Asendorpf (Eds.), Social withdrawal, inhibition, and shyness in childhood (pp. 265-289). Hillsdale: Erlbaum.

Asendorpf, J. (1994). The malleability of behavioral inhibition: A study of individual developmental functions. Developmental Psychology, 30, 912-919.

Balaban, M. T. \& Taussig, H. N. (1995). Salience of fear/threat in the affective modulation of the human startle blink. Biological Psychology, 38, 117-131.

Barrett, P., Rapee, R., Dadds, M. R., \& Ryan, S. M. (1996). Family enhancement of cognitive style in anxious and aggressive children: Threat bias and the FEAR effect. Journal of Abnormal Child Psychology, 24, 187-203. 


\section{REFERENCES}

Bates, J.E., Freeland, C., \& Lounsbury, M.L. (1979). Measurement of infant difficultness. Child Development, 50, 794-803.

Bernstein, G.A., Borchardt, C.M., \& Perwien, A.R. (1996). Anxiety disorders in children and adolescents: a review of the past 10 years. Journal of the American Academy of Child and Adolescent Psychiatry, 9, $1110-1119$.

Biederman, J., Rosenbaum, J.F., Hirshfeld, D.R., Faraone, S.V., Bolduc, E.A., Gersten, M., Meminger, S.R., Kagan, J., Snidman, N., \& Reznick, J.S. (1990). Psychiatric correlates of behavioral inhibition in young children of parents with and without psychiatric disorders. Archives of General Psychiatry, 47, $21-26$.

Biederman, J., Rosenbaum, J.F., Bolduc, E.A., Faraone, S.V., \& Hirshfeld, D.R. (1991). A high risk study of young children of parents with panic disorder and agoraphobia with and without comorbid major depression. Journal of Psychiatric Research, 37, 333-348.

Biederman, J., Rosenbaum, J.F., Bolduc-Murphy, E.A., \& Faraone, S.V. (1993). A three year follow-up of children with and without behavioral inhibition. Journal of the American Academy of Child and Adolescent Psychiatry, 32, 814-821.

Biederman, J., Rosenbaum, J.F., Chaloff, J., \& Kagan, J. (1995). Behavioral inhibition as a risk factor. In J.S. March (Ed.), Anxiety disorders in children and adolescents (pp. 61-81). New York: Guilford Press.

Birmaher, B., Brent, D.A., Chiappetta, L., Bridge, J., Monga, S., \& Baugher, M. (1999). Psychometric properties of the Screen for Child Anxiety Related Disorders (SCARED): A replication study. Journal of the American Academy of Child and Adolescent Psychiatry, 38, 1230-1236.

Birmaher, B., Khetarpal, S., Brent, D., Cully, M., Balach, M., Kaufman, J., et al. (1997). The Screen for Child Anxiety Related Emotional Disorders (SCARED): Scale construction and psychometric characteristics. Journal of the American Academy of Child and Adolescent Psychiatry, 36, 545-553.

Bishop, G., Spence, S.H., \& McDonald, C. (2003). Can parents and teachers provide a reliable and valid report of behavioral inhibition? Child Development, 74, 18991917.

Bögels, S.M., Van Oosten, A., Muris, P., \& Smulders, D. (2001). Familial correlates of childhood social anxiety. Behaviour Research and Therapy, 39, 273-287.

Bradley, M. M., Cuthbert, B. N., \& Lang, P. J. (1990). Startle reflex modification: emotion or attention? Psychophysiology, 27, 513-522.

Broberg, A.G. (1993). Inhibition and children's experiences of out-of-home care. In K.H. Rubin \& J. Asendorpf (Eds.), Social withdrawal, inhibition, and shyness in childhood (pp. 151-176). Hillsdale: Erlbaum. 
Brozina, K., \& Abela, J. R. Z. (2006). Behavioural inhibition, anxious symptoms, and depressive symptoms: A short-term prospective examination of a diathesis-stress model. Behaviour Research and Therapy, 44, 1337-1346.

Buss, A.H., \& Plomin, R. (1984). Temperament: Early developing personality traits._Hillsdale, N.J.: Erlbaum.

Calkins, S., \& Fox, N. (1992). The relations among infant temperament, security of attachment, and behavioral inhibition at twenty-four months. Child Development, 63, 14561472.

Calkins, S.D., Fox, N.A., \& Marshall, T.R. (1996). Behavioral and physiological antecedents of inhibited and uninhibited behavior. Child Development, 67, 523-540.

Caspi, A., Henry, B., McGee, R.O., Moffitt, T.E., \& Silva, P.A. (1995). Temperamental origins of child and adolescent behavior problems: From age three to age fifteen. Child Development, 66, 55-68.

Castro, J., Toro, J., van der Ende, J., \& Arrindell, W. A. (1993). Exploring the feasibility of assessing perceived parental rearing styles in Spanish children with the EMBU. International Journal of Social Psychiatry, 39, 47-57.

Cole, D.A., Peeke, L.G., Martin, J.M., Truglio, R., \& Seroczynski, A.D. (1998). A longitudinal look at the relation between depression and anxiety in children and adolescents. Journal of Consulting and Clinical Psychology, 66, 451-460.

Cook, E. W., III, Hawk, L. W., Davis, T. L., \& Stevenson, V. E. (1991). Affective individual differences and startle reflex modulation. Journal of Abnormal Psychology, 100, 513.

Cook, E. W., III, Hawk, L. W., Hawk, T. M., \& Hummer, K. (1995). Affective modulation of startle in children [Abstract]. Psychophysiology, 33 (Suppl. 1), S31.

Cooper, P.J., \& Eke, M. (1999). Childhood shyness and maternal social phobia: a community study. British Journal of Psychiatry, 174, 439-443.

Costello, E.J., \& Angold, A. (1995). Epidemiology. In J.S. March (Ed.), Anxiety disorders in children and adolescents (pp. 109-124). New York: Guilford Press.

Craske, M.G. (1997). Fear and anxiety in children and adolescents. Bulletin of the Menninger Clinic, 61 (Suppl. A), A4-A36.

Davidson, R.J. (1993). Childhood temperament and cerebral asymmetry: a neurobiological substrate of behavioral inhibition. In K.H. Rubin \& J. Asendorpf (Eds.), Social withdrawal, inhibition, and shyness in childhood (pp. 31 -48). Hillsdale: Erlbaum.

DiLalla, L.F., Kagan, J., \& Reznick, J.S, (1994). Genetic etiology of behavioral inhibition among 2-year-old children. Infant Behavior and Development, 17, 405-412.

Doornen, L. van (2000). Stress: Mind the body. De Psycholoog, 3, 114-118. 


\section{REFERENCES}

Ekman, P., \& Friesen, W. V. (1975). Unmasking the face: A guide to recognizing emotions from facial clues. Oxford: Prentice-Hall.

Ellis, L.K., \& Rothbart, M.K. (2001). Revision of the Early Adolescent Temperament Questionnaire. Poster presented at the Biennial Meeting of the Society for Research in Child Development. Minneapolis, Minnesota.

Epstein, S. (1979). The stability of behavior: I. On predicting most of the people much of the time. Journal of Personality and Social Psychology, 37, 1097-1126.

Epstein, S. (1980). The stability of behavior: II. Implications for psychological research. American Psychologist, 35, 790-806.

Fordham, K., \& Stevenson-Hinde, J. (1999). Shyness, friendship quality, and adjustment during middle childhood. Journal of Child Psychology and Psychiatry, 40, 757-768.

Fox, N.A., \& Calkins, S.D. (1993). Pathways to aggression and social withdrawal: interactions among temperament, attachment, and regulation. In K.H. Rubin \& J. Asendorpf (Eds.), Social withdrawal, inhibition, and shyness in childhood (pp. 81-100). Hillsdale: Erlbaum.

Fox, N.A., Henderson, H.A., Marshall, P.J., Nichols, K.E., \& Ghera, M.M. (2005). Behavioral Inhibition: Linking biology and behaviour within a developmental framework. Annual Review of Psychology, 56, 235-262.

Fox, N., Nichols, K. E., Henderson, H. A., Rubin, K., Schmidt, L., Hamer, D., Ernst, M., \& Pine, D. S. (2005). Evidence for a gene-environment interaction in predicting behavioral inhibition in middle childhood. Psychological Science, 16, 921-926.

Fullard, W., McDevitt, S.C., \& Carey, W.B. (1978). Toddler Temperament Scale. Philadelphia: Temple University, Department of Educational Psychology.

Garcia Coll, C., Kagan, J., \& Reznick, J.S. (1984). Behavioral inhibition in young children. Child Development, 55, 1005-1019.

Gest, S.D. (1997). Behavioral inhibition: Stability and associations with adaptation from childhood to early adulthood. Journal of Personality and Social Psychology, 72, 467-475.

Gladstone, G. L., \& Parker, G. B. (2006). Is behavioral inhibition a risk factor for depression? Journal of Affective Disorders, 95, 85-94.

Gladstone, G. L., Parker, G. B., Mitchell, P. B., Wilhelm, K. A., \& Mahli, G. S. (2005). Relationship between self-reported childhood behavioural inhibition and liefetime anxiety disorders in a clinical sample. Depression and Anxiety, 22, 103-113.

Grillon, C., \& Ameli, R. (1998). Effects of threat and safety signals on startle during anticipation of aversive shocks, sounds, or airblasts. Journal of Psychophysiology, 12, 329-337. 
Grillon, C., Dierker, L., \& Merikangas, K. R. (1997). Startle modulation in children at risk for anxiety disorders and/or alcoholism. Journal of the American Academy of Child and Adolescent Pscychiatry, 36, 925-932.

Grüner, K., Muris, P., \& Merckelbach, H. (1999). The relationship between anxious rearing behaviours and anxiety disorders symptomatology in normal children. Journal of Behavior Therapy and Experimental Psychiatry, 30, 27-35.

Gullone, E, Ollendick, T. H., \& King, N. J. (2006). The role of attachment representation in the relationship between depressive symptomatology and social withdrawal in middle childhood. Journal of Child and Family Studies, 15, 271-285.

Hagekull, B., \& Bohlin, G. (1995). Day care quality, family and child characteristics and socioemotional development. Early Childhood Research Quarterly, 10, 505-526.

Hayward, C., Killen, J.D., Kraemer, H.C., \& Taylor, C.B. (1998). Linking self-reported childhood behavioral inhibition to adolescent social phobia. Journal of the American Academy of Child and Adolescent Psychiatry, 37, 1308-1316.

Hazan, C., \& Shaver, P. (1987). Romantic love conceptualized as an attachment process. Journal of Personality and Social Psychology, 52, 511 -524.

Hirshfeld-Becker, D. R., Biederman, J., Calltharp, S., Rosenbaum, E. D., Faraone, S. V., \& Rosenbaum, S. V., (2003). Behavioral inhibition and disinhibition as hypothesized precursors to psychopathology: implications for pediatric bipolar disorder. Biological Psychiatry, 53, 985-999.

Hirshfeld-Becker, D.R., Biederman, J., \& Rosenbaum. J.F. (2004). Behavioral inhibition. In T.L. Morris \& J.S. March (Eds.), Anxiety disorders in children and adolescents, second edition (pp. 27-58). New York: Guilford Press.

Hirshfeld-Becker, D. R., Rosenbaum, J. F., Biederman, J. F., Bolduc, E. A., Faraone, S. V., Snidman, N., et al. (1992). Stable behavioral inhibition and its association with anxiety disorder. Journal of the American Academy of Child and Adolescent Psychiatry, 31, 103-111.

Hitchcock, J. M., \& Davis, M. (1986). Lesions of the amygdala, but not of the cerebellum or red nucleus, block conditioned fear as measured with the potentiated startle paradigm. Behavioral Neuroscience, 100, 11-22.

Hitchcock, J. M., \& Davis, M. (1987). Fear-potentiated startle using an auditory conditioned stimulus: Effect of lesions of the amygdala. Physiology and Behavior, 39, 403-408.

Hu, L., \& Bentler, P. M. (1999). Cutoff criteria for fit indexes in covariance structure analysis: conventional criteria versus new alternatives. Structural Equation Modeling, 6, 1-55.

Hudson, J. L., \& Rapee, R. M. (2001). Parent-child interactions and anxiety disorders: An observational study. Behaviour Research and Therapy, 39, 1411-1427. 


\section{REFERENCES}

Hudson J. L., \& Rapee, R. M. (2004). From anxious temperament to disorder: An etiological model of generalized anxiety disorder. In R. G. Heimberg, C. L. Turk, \& D. S. Mennin (Eds.), Generalized anxiety disorder: Advances in research and practice (pp. 51-74). New York: Guilford Press.

Jaffee, S. R., Moffitt, T. E., Caspi, A., Fombonne, E. P. R., \& Martin, J.(2002). Differences in early childhood risk factors for juvenile-onset and adult-onset depression. Archives of General Psychiatry, 59, 215-222.

Jöreskog K., \& Sörbom D. (1993). LISREL 8: structural equation modelling with the SIMPLIS command language. Lawrence Erlbaum, Hillsdale, NJ.

Kagan, J. (1989). The concept of behavioral inhibition to the unfamiliar. In J.S. Reznick (Ed.), Perspectives on behavioral inhibition (pp. 1-23). Chicago: The University of Chicago Press.

Kagan, J. (1994). Galen's prophecy: Temperament in human nature. New York: Basic Books.

Kagan, J., Reznick, J.S., \& Snidman, N. (1988). Biological bases of childhood shyness. Science, 240, 167-171.

Kagan, J., \& Snidman, N. (1991). Temperamental factors in human development. American Psychologist, 46, 856-862.

Kagan, J., Snidman, N., \& Arcus, D. (1993). On the temperamental categories of inhibited and uninhibited children. In K.H. Rubin \& J. Asendorpf (Eds.), Social withdrawal, inhibition, and shyness in childhood (pp. 19-28). Hillsdale: Erlbaum.

Kagan, J., Snidman, N., Zentner, M., \& Peterson, E. (1999). Infant temperament and anxious symptoms in school age children. Development and Psychopathology, 11, 209-224.

Kalin, N.H. (1997). The neurobiology of fear. Scientific American, 7 (special issue), 76-83.

Kerr, M., Lambert, W.W., Stattin, H., \& Klackenberg-Larsson, I. (1994). Stability of inhibition in a Swedish longitudinal sample. Child Development, 65, 138-146.

Kochanska, G., Aksan, N., Knaack, A., \& Rhines, H. M. (2004). Maternal parenting and children's conscience: Early security as moderator. Child Development, 75, 1229 1242.

Kotler, J.C., \& McMahon, R.J. (2002). Differentiating anxious, aggressive, and socially competent preschool children: Validation of the social competence and behavior evaluation-30 (parent version). Behaviour Research and Therapy, 40, 947-959.

LaFreniere, P.J., \& Dumas, J.E. (1995). Social Competence and Behavior Evaluation. (preschool ed.). Los Angeles: Western Psychological Services.

LaFreniere, P.J., \& Dumas, J.E. (1996). Social competence and behavior evaluation in children ages 3 to 6 years: The short form (SCBE-30). Psychological Assessment, 8, 369377.

Lang, P. J. (1995). The emotion probe. American Psychologist, 50, 372-385. 
Lang, P. J., Öhman, A., \& Vaitl, D. (1988). The International Affective Picture System [photographic slides]. The Center for Research in Psychophysiology, University of Florida, Gainesville, Fl.

Last, C.G., Perrin, S., Hersen, M., \& Kazdin, A.E. (1996). A prospective study of childhood anxiety disorders. Journal of the American Academy of Child and Adolescent Psychiatry, 35, 1502-1510.

LeDoux, J.E. (1997). Emotion, memory and the brain. Scientific American, 7 (special issue), 6875.

Lemery, K.S., Essex, M.J., \& Smider, N.A. (2002). Revealing the relation between temperament and behavior problem symptoms by eliminating measurement confounding: expert ratings and factor analyses. Child Development, 73, 867-882.

Liu, D., Diorio, J., Tannenbaum, B., Caldji, C., Francis, D., Freedman, A., Sharma, S., Pearson, D., Plotsky, P.M., \& Meaney, M. (1997). Maternal care, hippocampal glucocorticoid receptors, and hypothalamic-pituitary-adrenal responses to stress. Science, 277, 1659-1662.

Lonigan, C.J., \& Phillips, B.M. (2001). Temperamental influences on the development of anxiety disorders. In M.W. Vasey \& M.R. Dadds (Eds.), The developmental psychopathology of anxiety (pp.60-91). New York: Oxford University Press.

Manassis, K., Bradley, S., Goldberg, S., Hood, J., \& Swinson, R.P. (1994). Attachment in mothers with anxiety disorders and their children. Journal of the American Academy of Child and Adolescent Psychiatry, 33, 1106-1113.

Manassis, K., Bradley, S., Goldberg, S., Hood, J., \& Swinson, R.P. (1995). Behavioral inhibition, attachment and anxiety in children of mothers with anxiety disorders. Canadian Journal of Psychiatry, 40, 87-92.

March, J. S. (1995). Anxiety disorders in children and adolescents. New York: Guilford Press.

Marshall, P.J., \& Stevenson-Hinde, J. (1998). Behavioral inhibition, heart period, and respiratory sinus arrhythmia in young children. Developmental Psychobiology, 33, 283-292.

McManis, M. H., Bradley, M. M., Cuthbert, B. N., \& Lang, P. J. (1995). Kids have feelings too: Children's physiological responses to affective pictures [Abstract]. Psychophysiology, 33 (Suppl. 1), S53.

Meng, X.L., Rosenthal, R., \& Rubin, D.B. (1992). Comparing correlated correlation coefficients. Psychological Bulletin, 117, 172-175.

Merikangas, K. R., Avenevoli, S., Dierker, L., \& Grillon, C. (1999). Vulnerability factors among children at risk for anxiety disorders. Society of Biological Psychiatry, 46, 1523-1535.

Mick, M.A., \& Telch, M.J. (1998). Social anxiety and history of behavioral inhibition in young adults. Journal of Anxiety Disorders, 12, 1-20. 


\section{REFERENCES}

Muris, P. (2006). The pathogenesis of childhood anxiety disorders: Considerations from a developmental psychopathology perspective. International Journal of Behavioral Development, 30, 5-11.

Muris, P., Bosma, H., Meesters, C., \& Schouten, E. (1998). Perceived parental rearing behaviours: a confirmatory factor analytic study of the Dutch EMBU for children. Personality and Individual Differences, 24, 439-442.

Muris, P., \& Dietvorst, R. (2006). Underlying perspnality characteristics of behavioral inhibition in children. Child Psychiatry, 36, 437-445.

Muris, P., Dreessen, L., Bögels, S.M., Weckx, M. \& Van Melick, M. (2004). A questionnaire for screening a broad range of DSM-defined anxiety disorder symptoms in clinically referred children and adolescents. Journal of Child Psychology and Psychiatry, 45, 813-820.

Muris, P., Mayer, B., \& Meesters, C. (2000). Self-reported attachment style, anxiety, and depression in children. Social Behavior and Personality, 28, 157-162.

Muris, P., \& Meesters, C. (2002). Symptoms of anxiety disorders and teacher-reported school functioning of normal children. Psychological Reports, 91, 588-590.

Muris, P., Meesters, C., \& Blijlevens, P. (submitted). Self-reported reactive and regulative temperament in middle childhood: Relations to the Big Three and internalizing and externalizing problem behavior.

Muris, P., Meesters, C., \& Mayer, B. (2000). The Koala Fear Questionnaire: A standardized, pictorial scale for measuring fears in young children. Maastricht: Maastricht University.

Muris, P., Meesters, C., Mayer, B., Bogie, N., Luijten, M., Geebelen, E., Bessems, J. \& Smit, C. (2003). The Koala Fear Questionnaire: A standardized self-report scale for assessing fears and fearfulness in pre-school and primary school children. Behaviour Research and Therapy, 41, 597-617.

Muris, P., Meesters, C., Merckelbach, H., \& Hülsenbeck, P. (2000). Worry in children is related to perceived parental rearing and attachment. Behavior Research and Therapy, 38, 487-497.

Muris, P., Meesters, C., \& Spinder, M. (2003). Relationships between child-and parent-reported behavioral inhibition and symptoms of anxiety and depression in normal adolescents. Personality and Individual Differences, 34, 759-771.

Muris, P., Meesters, C., \& van Brakel, A. (2003). Assessment of anxious Rearing Behaviors with a modified version of "Egna Minnen Beträffande Uppfostran" questionnaire for children. Journal of Psychopathology and Behavioral Assessment, 25, 229-237. 
Muris, P., Meesters, C., van Melick, M., \& Zwambag, L. (2001). Self-reported attachment style, attachment quality, and symptoms of anxiety and depression in young adolescents. Personality and Individual Differences, 30, 809-818.

Muris, P., Merckelbach, H., Kindt, M., Bögels, S., Dreessen, L., Van Dorp, C., Habets, A., Rosmuller, S., \& Snieder, N. (2001). The utility of screen for child anxiety related emotional disorders (SCARED) as a tool for identifying children at high risk for prevalent anxiety disorders. Anxiety, Stress, and Coping, 14, 265-283.

Muris, P., Merckelbach, H., Wessel, I., \& Van de Ven, M. (1999). Psychopathological correlates of self-reported behavioral inhibition in normal children. Behaviour Research and Therapy, 37, 575-584.

Muris, P., Merkelbach, H., Schmidt, H., Gadet, B., \& Bogie, N. (2001). Anxiety and depression as correlates of self-reported behavioral inhibition in normal adolescents. Behaviour Research and Therapy, 39,1051-1061.

Muris, P., \& Ollendick, T. H. (2005). The role of temperament in the etiology of child psychopathology. Clinical Child and Family Psychology Review, 8, 271-289.

Muris, P., Schmidt, H., \& Merckelbach, H. (2000). Correlations among two self-report questionnaires for measuring DSM-defined anxiety disorder symptoms in children: The Screen for Child Anxiety Related Emotional Disorders and the Spence Children's Anxiety Scale. Personality and Individual Differences, 28, 333-346.

Murphy, K.R., \& Davidshofer, C.O. (1994). Psychological testing. Principles and applications. Englewood Cliffs: Prentice Hall.

Neal, J. A., \& Edelmann, R. J. (2003). The etiology of social phobia: Toward a developmental profile. Clinical Psychology Review, 23, 761-786.

Ornitz, E. M. (1999). Startle modification in children and developmental effects. In M. E. Dawson, A. M. Schell, \& A. H. Böhmelt (Eds.), Startle modification: implications for neuroscience, cognitive science, and clinical science (pp.245-266). Cambridge: Cambridge University Press.

Partridge, T (2000). Temperament development modeled as a nonlinear complex adaptive system. Nonlinear Dynamics in Psychology and Life Sciences, 4, 339-357.

Pollack, M.H., Otto, M.W., Rosenbaum, J.F., \& Sachs, G.S. (1992). Personality disorders in patients with panic disorder: Association with childhood anxiety disorders, early trauma, comorbidity, and chronicity. Comprehensive Psychiatry, 29, 72-75.

Rapee, R.M. (1997). Potential role of childrearing practices in the development of anxiety and depression. Clinical Psychology Review, 17, 47-67.

Rapee, R. M., \& Spence, S. H. (2004). The etiology of social phobia: Empirical evidence and an initial model. Clinical Psychology Review, 24, 737-767. 


\section{REFERENCES}

Rapee, R. M., Kennedy, S., Ingram, M., Edwards, S., \& Sweeney, L. (2005). Prevention and early intervention of anxiety disorders in inhibited preschool children. Journal of Consulting and Clinical Psychology, 73, 488-497.

Reznick, J.S. (1989). Perspectives on behavioral inhibition. Chicago: The University of Chicago Press

Reznick, J.S., Gibbons, J.L., Johnson, M.O., \& McDonough, P.M., (1989). Behavioral inhibition in a normative sample. In J.S. Reznick (Ed.), Perspectives on behavioral inhibition (pp. 25-49). Chicago: The University of Chicago Press.

Reznick, J.S., Hegeman, I.M., Kaufman, E.R., \& Woods, S.W. (1992). Retrospective and concurrent self-report of behavioral inhibition and their relation to adult mental health. Development and Psychopathology, 4, 301-321.

Robinson, J.L., Kagan, J., Reznick, J.S., \& Corley, R. (1992). The heritability of inhibited and uninhibited behavior: A twin study. Developmental Psychology, 28, 1030-1037.

Rosenbaum, J.F., Biederman, J., Gersten, M., Hirshfeld, D.R., Meminger, S.R., Herman, J.B., Kagan, J., Reznick, J.S., \& Snidman, N. (1988). Behavioral inhibition in children of parents with panic disorder and agoraphobia: a controlled study. Archives of General Psychiatry, 45, 463-470.

Rosenbaum, J.F., Biederman, J., Hirshfeld, D.R., Bolduc, E.A., Faraone, S.V., Kagan, J., Snidman, N., \& Reznick, J.S. (1991). Further evidence of an association between behavioral inhibition and anxiety disorders: results from a family study of children from a non-clinical sample. Journal of Psychiatric Research, 25, 49-65.

Rosenbaum, J.F., Biederman, J., Bolduc, E.A., Hirshfeld, D.R., Faraone, S.V., \& Kagan, J. (1992). Comorbidity of parental anxiety disorders as risk for childhood-onset anxiety in inhibited children. American Journal of Psychiatry, 149, 475-481.

Rosenbaum, J.F., Biederman, J., Bolduc-Murphy, E.A., Faraone, S.V., Chaloff, J., Hirshfeld, D.R., \& Kagan, J. (1993). Behavioral inhibition in childhood: a risk factor for anxiety disorders. Harvard Review of Psychiatry, 1, 2-16.

Rosenbaum, J.F., Biederman, J., Pollock, R.A., \& Hirshfeld, D.R. (1994). The etiology of social phobia. Journal of Clinical Psychiatry, 55, 10-16.

Rothbart, M.K. (1981). Measurement of temperament in infancy. Child Development, 52, 569 578.

Rothbart, M. K., Ellis, L. K., \& Posner, M. I. (2004). Temperament and self-regulation. In Baumeister R. F., \& Vohs, K. D. (eds) Handbook of self-regulation. Research, theory, and applications, Guilford Press, New York, pp. 357-370.

Rubin, K.H., Hastings, P.D., Stewart, S.L., Henderson, H.A., \& Chen, X. (1997). The consistency and concomitants of inhibition: Some of the children, all of the time. Child Development, 68, 467-483. 
Rubin, K.H., Nelson, L.J., Hastings, P., \& Asendorpf, J. (1999). The transaction between parents' perceptions of their children's shyness and their parenting styles. International Journal of Behavioral Development, 23, 937-957.

Rubin, K.H., \& Mills, R.S.L. (1991). Conceptualizing developmental pathways to internalizing disorders in childhood. Canadian Journal of Behavioral Science, 23, 300-317.

Sapolsky, R.M. (1997). The importance of a well-groomed child. Science, 277, 1620-1621.

Sarason, I. G., Johnson, J. H. \& Siegel, J. M. (1978). Assessing the impact of life changes: Development of the Life Experiences Survey. Journal of Consulting and Clinical Psychology, 46, 932-946.

Scarpa, A., Raine, A., Venables, P.H., \& Mednick, S.A. (1995). The stability of inhibited/uninhibited temperament from ages 3 to 11 years in Mauritian children. Journal of Abnormal Child Psychology, 23, 607-618.

Schmidt, L. A., \& Fox, N. A. (1998). Fear-potentiated startle responses in temperamentally different human infants. Developmental Psychobiology, 32, 113-120.

Schmidt, L.A., Fox, N.A., Rubin, K.H., Sternberg, E.M., Gold, P.W., Smith, C.C., \& Schulkin, J. (1997). Behavioral and neuroendocrine responses in shy children. Developmental Psychobiology, 30, 127-140.

Schmidt, L. A., Fox, N. A., Schulkin, J., \& Gold, P. W. (1999). Behavioral and psychophysiological correlates of self-presentation in temperamentally shy children. Developmental Psychobiology, 35, 119-135.

Schmitz, S., Fulker, D.W., Plomin, R., Zahn-Waxler, C., Emde, R.N., \& DeFries, J.C. (1999). Temperament and problem behaviour during early childhood. International Journal of Behavioral Development, 23, 333-355.

Schwartz, C. E., Wright, C. I., Shin, L. M., Kagan, J., \& Rauch, S. L. (2003). Inhibited and uninhibited infants "grown up": adult amygdalar response to novelty. Science, 300, 1952-1953.

Schwartz, C. E., Wright, C. I., Shin, L. M., Kagan, J., Whalen, P. J., McMullin, K. G., \& Rauch, S. L. (2003). Differential amygdalar response to novel versus newly familiar neutral faces: a functional MRI probe developed for studying inhibited temperament. Society of Biological Psychiatry, 53, 854-862.

Scot, K. G., Mason, C. A., \& Chapmen, D. A. (1999). The use of epidemiological methodology as a means of influencing public policy. Child Development, 70, 1263-1272.

Shamir-Essakow, G., Ungerer, J.A. \& Rapee, R. M. (2005). Attachment, behavioral inhibition, and anxiety in preschool children. Journal of Abnormal Child Psychology, 33, 131 143.

Shatz, S. M. (2005). The psychometric properties of the behavioral inhibition scale in a collegeaged sample. Personality and Individual Differences, 39, 331-339. 


\section{REFERENCES}

Snidman, N. (1989). Behavioral inhibition and sympathetic influence on the cardiovascular system. In J.S. Reznick (Ed.), Perspectives on behavioral inhibition (pp. 51-70). Chicago: The University of Chicago Press.

Snidman, N., \& Kagan, J. (1994). The contribution of infant temperamental differences to acoustic startle response [Abstract]. Psychophysiology, 31, S92.

Spence, S.H. (2001). Prevention strategies. In M.W. Vasey \& M.R. Dadds (Eds.), The Developmental Psychopathology of Anxiety (pp. 325-351). Oxford: University Press.

Spielberger, C. D., Gorsuch, R. L., \& Lushene, R. E. (1970). STAI Manual for the State-Trait Inventory. Consulting Psychologists Press, Palo Alto, California.

Spielberger, C. D., Gorsuch, R. L., Lushene, R. E., Vagg, R. E., \& Jacobs, G. A. (1983). Manual for the State-Trait Anxiety Inventory. Consulting Psychologists Press, Palo Alto, California.

Stevenson-Hinde, J., \& Glover, A. (1996). Shy girls and boys: A new look. Journal of Child Psychology and Psychiatry, 37, $181-187$.

Stevenson-Hinde, J., \& Shouldice, A. (1995). 4.5 to 7 years: Fearful behavior, fears and worries. Journal of Child Psychology and Psychiatry, 36, 1027-1038.

Suomi, S.J. (1997). Early determinants of behaviour: Evidence from primate studies. British Medical Bulletin, 53, 170-184.

Thompson, R. A., \& Raikes, H. A. (2003). Toward the next quarter-century: Conceptual and methodological challenges for attachment theory. Development and Psychopathology, 15, 691-718.

Tiet, Q. Q., Bird, H. R., Hoven, C. W., Moore, R., Wu, P., Wicks, J., Jensen, P. S., Goodman, S., \& Cohen, P. (2001). Relationship between specific adverse life events and psychiatric disorders. Journal of Abnormal Child Psychology, 29, 153-164.

Townsley-Stemberger, R., Turner, S.M., Beidel, D.C., \& Calhoun, K.S. (1995). Social phobia: An analysis of possible developmental factors. Journal of Abnormal Psychology, 104, 526-531.

Turner, S. M., Beidel, D. C., Roberson-Nay, R., \& Tervo, K. (2003). Parenting behaviors in parents with anxiety disorders. Behaviour Research and Therapy, 41, 541-555.

Turner, S.M., Beidel, D.C., \& Wolff, P.L. (1996). Is behavioral inhibition related to the anxiety disorders? Clinical Psychology Review, 16, 157-172.

Valla, J. P., Bergeron, L., Bidaut-Rusell, M., St-Georges, M., \& Gaudet, M. (1997). Reliability of the Dominic-R: a young child mental health questionnaire combining visual and auditory stimuli. Journal of Child Psychology and Psychiatry, 38, 717-724.

Valla, J. P., Bergeron, L., \& Smolla, N. (2000). The Dominic-R: A pictorial interview for 6- to 11 -year old children. Journal of the American Academy for Child and Adolescent Psychiatry, 39, 85-93. 
Van Brakel, A. M. L., \& Muris, P. (2006). A brief scale for measuring "behavioral inhibition to the unfamiliar" in children. Journal of Psychopathology and Behavioral Assessment, 28, 79-84.

Van Brakel, A.M.L., Muris, P. \& Bögels, S. (2001). Gedragsinhibitie als risicofactor voor het ontwikkelen van angststoornissen bij kinderen: een overzicht. (Behavioral inhibition as a risk factor for the development of anxiety disorders in children: a review.) Nederlands Tijdschrift voor de Psychologie en haar grensgebieden, 56, 57-68.

Van Brakel, A.M.L., Muris, P., \& Bögels, S.M. (2004). Relations between parent- and teacherreported behavioral inhibition and behavioral observations of this temperamental trait. Journal of Clinical Child and Adolescent Psychology, 33, 579-589.

Van Brakel, A. M. L., Muris, P., Bögels, S. M., \& Thomassen, C. (2006). A multifactorial model for the etiology of anxiety in non-clinical adolescents: Main and interactive effects of behavioral inhibition, attachment, and parental rearing. Journal of Child and Family Studies, 15, 568-578.

Van der Ploeg, H. M. (1980). Validity of the Zelf-Beoordelings-Vragenlijst (A Dutch version of the Spielberger State-Trait Anxiety Inventory). Nederlands Tijdschrift voor de Psychologie en haar Grensgebieden, 35, 243-249.

Van der Ploeg, H. M., Defares, P. B., \& Spielberger, C. D. (1979). Een nederlandstalige bewerking van de Spielberger State-Trait Anxiety Inventory, STAI-DY. Handleiding bii de Zelf-Beoordelings-Vragenlijst. Swets \& Zeitlinger B.V.

Vasa, R. A., \& Pine, D. S. (2004). Neurobiology. In T. L. Morris and J. S. March (Eds.), Anxiety disorders in children and adolescents (pp. 3-26). New York: The Guilford Press.

Vasey, M. W., \& Dadds, M. R. (2001). An introduction to the developmental psychopathology of anxiety. In M.W. Vasey \& M.R. Dadds (Eds.), The developmental psychopathology of anxiety (pp. 3-26). Oxford: Oxford University Press.

Verhulst, F.C., Van der Ende, J., Ferdinand, R.F., \& Kasius, M.C. (1997) The prevalence of DSM-III-R diagnoses in a national sample of Dutch adolescents. Archives of General Psychiatry, 54, 329-336.

Warren, S.L., Huston, L., Egeland, B., \& Sroufe, L.A. (1997). Child and adolescent anxiety disorders and early attachment. Journal of the American Academy of Child and Adolescent Psychiatry, 36, 637-644.

Wenar, C., \& Kerig, P. (2000). Developmental psychopathology: From infancy through adolescence. New York: McGraw-Hill.

Westenberg, P.M., Siebelink, B. M., \& Treffers, P. D. A. (2001). Psychosocial developmental theory in relation to anxiety and its disorders. In W. K. Silverman \& P. D. A. Treffers (Eds.), Anxiety disorders in children (pp. 72-89). Cambridge: Cambridge University Press. 


\section{REFERENCES}

Whaley, S. E., Pinto, A., \& Sigman, M. (1999). Characterizing interactions between anxious mothers and their children. Journal of Consulting and Clinical Psychology, 67, 826836.

Wood, J. J., Mcleod, B. D., Sigman, M., Hwang, W-C., \& Chu, B. C. (2003). Parenting and childhood anxiety: theory, empirical findings, and future directions. Journal of Child Psychology and Psychiatry, 44, 134-151.

Woodward, S. A., McManis, M. H., Kagan, J., Deldin, P. Snidman, N., Lewis, M., \& Kahn, V. (2001). Infant temperament and the brainstem auditory evoked response in later childhood. Developmental Psychology, 37, 533-538. 


\section{DANKWOORD}

Deze professionele milpaal had ik niet kunnen bereiken zonder de medewerking van alle kinderen en hun ouders die deelgenomen hebben aan mijn onderzoek.

Lieve kinderen, dank jullie wel! Ik wens jullie onwijs veel wijsheid, moed en kracht toe op weg naar volwassenheid. Ik zal jullie nooit vergeten.

In alfabetische volgorde zijn jullie hier allemaal:

Alessandro, Alex, A. Wares, Amy, Amy, Amber, Ankie, Angelica, Ankie, Anne-Laura, Annemiek, Ard, Arianne, Arian, Bart, B. Kisoka, Bart, Bastiaan, Bettino, Bekka, Bianca, Branco, Bram, Bram, Bram, Brigitte, Carene, Caro, Caro, Carlos, Celine, Charlotte, Chelly, Chrissy, Claudia, Cyriel, Dana, Dahnee, Daphne, Danielle, Deafoon, Debbie, Dick, Diango, Dominic, Dominique, Eline, Elke, Elmo, Emma, Eloy, Emily, Eva, Eveline, Fabienne, Floris, Floris, Floor, Franca, Froukie, Glenn, Glenn, Gwenn, Ilona, Imke, Indy, Janou, Jans, Jason, Jasper, Jaron, Jelisej, Jemy, Jenny, Jesse, Jef, Jeffrey, Jelle, Jennifer, Jeroen, Jimmy, Jolien, Joas, Joey, Jolanda, Joliin, Jore, Joyce, Judi Joan, Jordi, Juliette, Koen, Krit, Kylie, Kenny, Kevin, Kevin, Kira, Kirsten, Kirsten, Koen, Lara, Lara, Larissa, Laura, Laura, Laura, Laura, Laura, Laurens, Leatitia, Lieke, Lisa, Linsey, Lizanne, Lotte, Lotte, Lotte, Louwie, Lucas, LuAnn, Luc, Lynn, Lynn, Lynn, Maarten, Macha, Maddy, Maikel, Maikel, Manouk, Mara, Marco, Mark, Mark, Mark, Maroel, Martiin, Maud, Maud, Maud, Max, Maxine, Melissa, Melissa, Michelle, Michiel, Miek, Minoesch, Mike, Mike, Mike, Milly, Milou, Mireille, Nadia, Nadiah, Naomi, Natalie, Nathalya, Naut, Nico, Nicole, Niek, Niels, Niels, Niki, Nikki, Nikkie, Nina, Nils, Noor, Noortje, Olivia, Onno, Pascal, Pascalle, Pascalle, Patrick, Pepiin, Quincy, Rachel, Rachel, Ray, Renee, Rens, Rens, Rick, Rick, Rik, Rik, Rinaldo, Risto, Ritchie, Robin, Robin, Roel, Romile, Romy, Romy, Rowan, Roxanne, Ruben, Ruben, Sabine, Safa, Salina, Samantha, Samyra, Sander, Sandy, Sanne, Sanne, Saskia, Selina, Selina, Sera, Sharine, Sharomir, Shawny, Simone, Simone, Simone, Shira, Siri, Sjoerd, Sophie, Stan, Stan, Stan, Stan, Stef, Stephanie, Steven, Susanne, Tammy, Tanja, Tavi, Tessa, Tessel, Thijs, Thijs, Tim, Tim, Tom, Tom, Tristan, Venice, Vera, Vera, Veronique, Vince, Wendy, Winnie, Wouter, Youri, Yves, Yves, Yvonne, Zenna, Zino, Zoe.

Prof. dr. Muris. Beste Peter, zonder jou zou mijn publicatielijst in de loop der jaren niet gegroeid, misschien zelfs wel non-existent zijn. Mijn ellenlange teksten werden ondanks deze 'onwetenschappelijke vitgebreidheid' door jou altiid met luid gejuich 
ontvangen. Tijdens het 'interne' review proces dat volgde werd mij steeds langzaam duidelijk wat er geschrapt moest worden en wat korter kon. Voor jou leek het misschien een never ending story, voor mij zeker een ontzettend goede leerschool. Grote dank voor dit rugzakje wetenschappelijke schrijfwijsheid! Dank ook voor je wijsheid in het versimpelen van, in mijn ogen, onoverkomelijke problemen tot simpele, te behappen kleinigheden. Ignore by deleting, how can I ever forget! En niet de minste dank voor de eindsprint......

Prof. dr. Arntz. Beste Arnoud, onze openhartige gesprekken op momenten dat ik het erg moeilijk had de afgelopen jaren zijn van onschatbare waarde geweest, met name voor mij persoonlijk, maar viteraard ook voor mijn promotietraject en de afronding hiervan in de vorm van dit proefschrift. In een voor mij moeilijke periode besloot iij om promotor te worden, waardoor je mij niet alleen uit een benauwde situatie haalde, maar ook aangaf dat je achter me stond. Je rust, je ruimte, en je oprechte aandacht en bezorgdheid hebben mij gesteund en een hoop geleerd. Dank daarvoor! En viteraard ook voor die supersnelle eindsprint.....

Mevr. Claessens. Beste Astrid, ik weet dat je nu denkt 'hoezo sta ik in haar dankwoord, ze heeft alles zelf gedaan'. Dit is namelijk de kern van wat ik van jou geleerd hebt. '...Je doet alles zelf, je dient je verantwoordelijkheid zelf te nemen, maar je dient ook zelf bij anderen een antwoord, een daad, een actie o.i.d. te ontlokken. Wacht nooit af!...' Ik glimlach nu ik dit opschrijf. Wat waren de gesprekken met jou verhelderend. En wat heb ik een hoop over mezelf geleerd. Astrid, ik kan niet anders zeggen dan dat iij mijn rots in de branding bent geweest op cruciale momenten tijdens mijn promotietraject. Ik weet dat je het niet wil horen, maar zonder jou was ik echt kopje onder gegaan! Duizendmaal dank!!

Erik, statistische steun en toeverlaat voor zovelen, inclusief moi. Geduld is een gave en iii hebt 'm! Dank daarvoor!

Een aantal studenten hebben in het kader van hun studie meegewerkt aan de totstandkoming van mijn onderzoek. Vier van hen wil ik speciaal benoemen: Wendy, Charlotte, Melissa en Pomme. Dank jullie wel voor jullie ondersteuning, enthousiasme en persoonlijke betrokkenheid.

Dank ook aan mijn ex-collega's van het Departement Medische, Klinische en Experimentele Psychologie. De volgende personen, ieder op hun eigen manier, in 
het bijzonder: Angela, Annie, Charlie, Cor, Emmy, Jeanine, Josephine, Lindy, Mark, Nicole B., Nicole D., en Theo.

Truus, door jou zijn in de laatste fase van mijn onderzoek alle 'uit- en invoerende puntjes' op de i gezet. Dank hiervoor! Dank ook voor het vitwisselen van ideeën, suggesties en gevoelens zowel op professioneel als persoonlijk vlak.

Last but not least, twee hele speciale ex-collega's: Guus Smeets en Maaike Cima. Lieve Guus, wat heb ik een hoop van je geleerd!! Wij samen op 1 kamer, ik zal het nooit vergeten. Lieve Maaike, tijdens onze studie hebben we een kans gehad, maar niet genomen. Nu doen we het dubbel en dwars over...en nog meer. Lieve alletwee, geweldig dat ik jullie heb mogen leren kennen. Super thanks dat jullie als paranimfen naast me willen staan.

Uiteraard ook een dikke zoen voor al mijn vriendinnen en vrienden aan de zijlijn, zonder jullie afleiding, ontspanning en coaching was de weg naar de finish stukken minder leuk geweest!

Beste Remy, zonder jou had dit proefschrift er echt niet zo mooi vitgezien. Duizendmaal dank hiervoor, maar ook voor de rust die je (of eigenlijk ik door jou) kon bewaren ondanks de tijdsdruk.

Lieve Mama, met name door jou ben ik geworden wie ik nu ben. Je bent een moedige vrouw en mijn voorbeeld in het tonen van kracht, doorzettingsvermogen, flexibiliteit en veerkracht. Woorden van dank zouden hier tekort doen.

Lieve Carolien, ons leven kent diepe dalen en hoge toppen, maar we komen er wel. Thanks for being there!

Allerliefste Sjoerd, Anna Sofie en Eva Louise, wat een voorrecht om bij jullie te mogen horen! Jullie geven glans aan mijn bestaan. Dank voor jullie onvoorwaardelijke steun, liefde en eindeloze geduld. Op naar onze volgende vitdaging: Uganda here we come! xxx 



\section{CURRICULUM VITAE}

Anna Maria Louisa van Brakel werd op 8 februari 1976 geboren in Tilburg. In 1994 behaalde zij het VWO-diploma aan het Cobbenhagen College te Tilburg. In januari 1995 vertrok zij naar Uganda (Afrika) om zes maanden in een tweetal weeshuizen en aan een basisschool aldaar te werken. Vanaf augustus 1995 studeerde zii Psychologie aan de Universiteit Maastricht met als afstudeerrichting Neuropsychologie. In het kader van haar afstudeerstage heeft zij in 1999 zes maanden onderzoek gedaan aan de Universiteit van Lund (Zweden). Per februari 2000 werd zii parttime aangesteld als Assistent in Opleiding ( $\mathrm{AiO}$ ) en parttime als Toegevoegd Docent (ToDo) bij het Departement Medische, Klinische en Experimentele Psychologie (thans Clinical Psychological Science) aan de Universiteit Maastricht. Het onderzoek beschreven in dit proefschrift werd gedurende deze aanstelling uitgevoerd. Vanaf oktober 2007 zal zij woonachtig en werkzaam zijn in Uganda (Afrika). 



\section{LIST OF PUBLICATIONS}

Van Brakel, A. M. L., \& Muris, P. (2006). A brief scale for measuring "behavioral inhibition to the unfamiliar" in children. Journal of Psychopathology and Behavioral Assessment, 28, 79-84.

Van Brakel, A. M. L., Muris, P., Arntz, \& Schouten, E. (submitted). Behavioral inhibition as a risk factor for the development of childhood anxiety disorders: A longitudinal study.

Van Brakel, A. M. L., Muris, P., \& Bögels, S. M. (2004). Relations between parent- and teacher-reported behavioral inhibition and behavioral observations of this temperamental trait. Journal of Clinical Child and Adolescent Psychology, 33, 579-589.

Van Brakel, A. M. L., Muris, P., \& Bögels, S. M. (2001). Gedragsinhibitie als risicofactor voor het ontwikkelen van angststoornissen bij kinderen: een overzicht.(Behavioral inhibition as a risk factor for the development of anxiety disorders in children:a review). Nederlands Tiidschrift voor de Psychologie en haar grensgebieden, 56, 57-68.

Van Brakel, A. M. L., Muris, P., Bögels, S. M. \& Thomassen, C. (2006). A multifactorial model for the etiology of anxiety in non-clinical adolescents: Main and interactive effects of behavioural inhibition, attachment, and parental rearing. Journal of Child and Family Studies, 15, 568-578.

Van Brakel, A. M. L., Muris, P. \& Derks, W. (2006). Eye blink startle responses in behaviorally inhibited and uninhibited children. International Journal of Behavioral Development, 30, 70-75.

Muris, P., Meesters, C. \& Van Brakel, A. (2003). Assessment of anxious rearing behaviors with a modified version of the "Egna Minnen Beträffande Uppfostran" (EMBU) questionnaire for children. Journal of Psychopathology and Behavioral Assessment, 25, 229-237.

Muris, P., Merckelbach, H., Mayer, B., Van Brakel, A., Thissen, S., Moulaert, V. \& Gadet, B. (1998). The Screen for Child Anxiety Related Emotional Disorders and its relationship to traditional childhood anxiety measures. Journal of Behavior Therapy and Experimental Psychiatry, 29, 327-339. 
Muris, P., Merckelbach, H., Van Brakel, A. \& Mayer, B. (1999). The revised version of the Screen for Child Anxiety Related Emotional Disorders (SCARED-R): Further evidence for its reliability and validity. Anxiety, Stress, and Coping, 12, 411-425.

Muris, P., Merckelbach, H., Van Brakel, A., Mayer, B. \& Dongen, L. van (1998). The Screen for Child Anxiety Related Emotional Disorders: Relationship with anxiety and depression in normal children. Personality and Individual Differences, 24, 452-456.

Muris, P., Van Brakel, A. \& Meesters, C. (1998). Coping styles, anxiety, and depression in children. Psychological Reports, 83, 1225-1226.

Rassin, E., Van Brakel, A. \& Diederen, E. (2003). Suppressing unwanted memories: Where there is a will, there is a way? Behaviour Research and Therapy, 41, 727-736. 